NIST Special Publication 941

\title{
Free Trade Area of the Americas (FTAA) Conformity Assessment Infrastructure
}

\section{Carmiña Londoño}

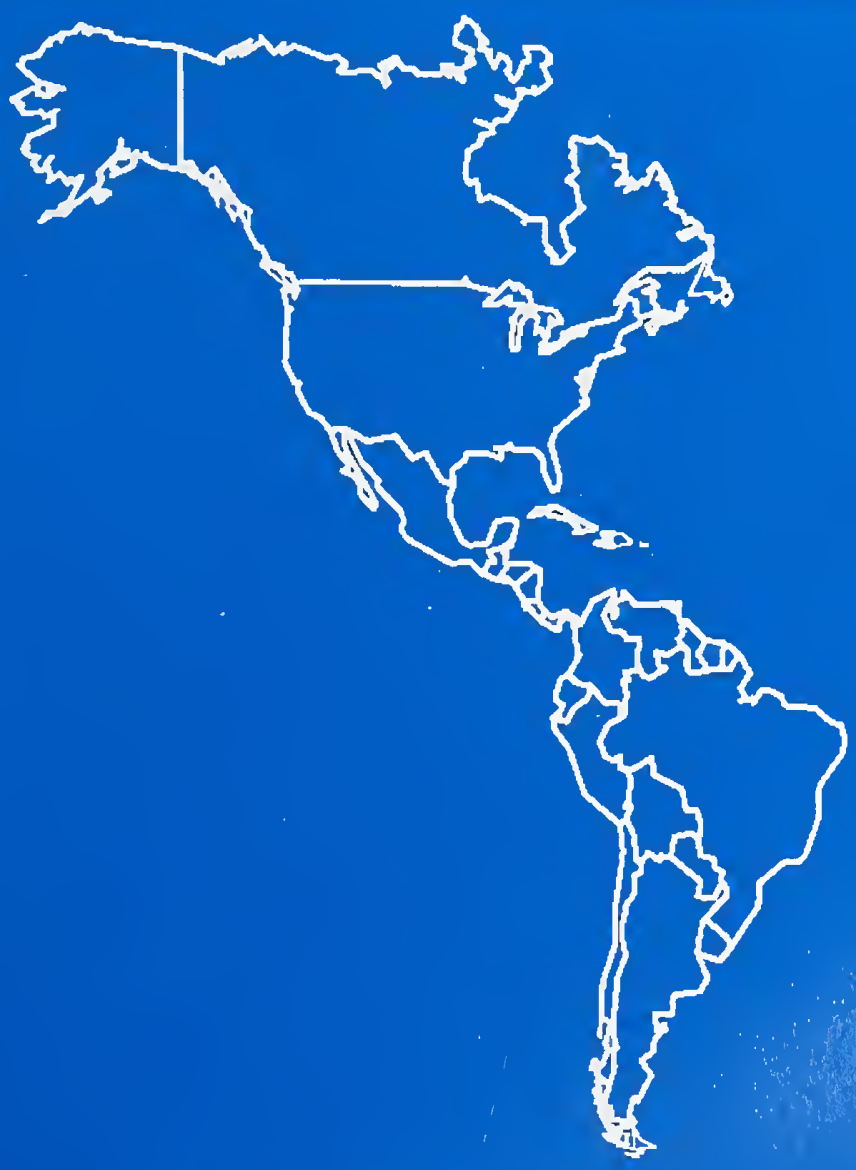


he National Institute of Standards and Technology was established in 1988 by Congress to "assist industry in the development of technology ... needed to improve product quality, to modernize manufacturing processes, to ensure product reliability ... and to facilitate rapid commercialization ... of products based on new scientific discoveries."

NIST, originally founded as the National Bureau of Standards in 1901, works to strengthen U.S. industry's competitiveness; advance science and engineering; and improve public health, safety, and the environment. One of the agency's basic functions is to develop, maintain, and retain custody of the national standards of measurement, and provide the means and methods for comparing standards used in science, engineering, manufacturing, commerce, industry, and education with the standards adopted or recognized by the Federal Government.

As an agency of the U.S. Commerce Department's Technology Administration, NIST conducts basic and applied research in the physical sciences and engineering, and develops measurement techniques, test methods, standards, and related services. The Institute does generic and precompetitive work on new and advanced technologies. NIST's research facilities are located at Gaithersburg, MD 20899, and at Boulder, CO 80303. Major technical operating units and their principal activities are listed below. For more information contact the Publications and Program Inquiries Desk, 301-975-3058.

\section{Office of the Director}

- National Quality Program

- International and Academic Affairs

\section{Technology Services}

- Standards Services

- Technology Partnerships

- Measurement Services

- Technology Innovation

- Information Services

\section{Advanced Technology Program}

- Economic Assessment

- Information Technology and Applications

- Chemical and Biomedical Technology

- Materials and Manufacturing Technology

- Electronics and Photonics Technology

\section{Manufacturing Extension Partnership Program \\ - Regional Programs \\ - National Programs \\ - Program Development}

\section{Electronics and Electrical Engineering}

Laboratory

- Microelectronics

- Law Enforcement Standards

- Electricity

- Semiconductor Electronics

- Electromagnetic Fields ${ }^{1}$

- Electromagnetic Technology'

- Optoelectronics ${ }^{1}$

\section{Chemical Science and Technology Laboratory \\ - Biotechnology \\ - Physical and Chemical Properties ${ }^{2}$ \\ - Analytical Chemistry \\ - Process Measurements \\ - Surface and Microanalysis Science}

Physics Laboratory

- Electron and Optical Physics

- Atomic Physics

- Optical Technology

- Ionizing Radiation

- Time and Frequency ${ }^{1}$

- Quantum Physics ${ }^{1}$

Materials Science and Engineering Laboratory

- Intelligent Processing of Materials

- Ceramics

- Materials Reliability'

- Polymers

- Metallurgy

- NIST Center for Neutron Research

Manufacturing Engineering Laboratory

- Precision Engineering

- Automated Production Technology

- Intelligent Systems

- Fabrication Technology

- Manufacturing Systems Integration

\section{Building and Fire Research Laboratory \\ - Structures \\ - Building Materials \\ - Building Environment \\ - Fire Safety Engineering \\ - Fire Science}

\section{Information Technology Laboratory}

- Mathematical and Computational Sciences ${ }^{2}$

- Advanced Network Technologies

- Computer Security

- Information Access and User Interfaces

- High Performance Systems and Services

- Distributed Computing and Information Services

- Software Diagnostics and Conformance Testing

- Statistical Engineering

\footnotetext{
'At Boulder, CO 80303.

${ }^{2}$ Some elements at Boulder, $\mathrm{CO}$.
} 


\section{NIST Special Publication 941}

\section{Free Trade Area of the Americas (FTAA) Conformity Assessment Infrastructure}

Carmiña Londoño

Office of Standards Services

Global Standards Program

National Institute of Standards and Technology

Gaithersburg, MD 20899-0001

July 1999

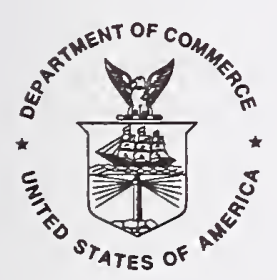

U.S. Department of Commerce

William M. Daley, Secretary

Technology Administration

Gary R. Bachula, Acting Under Secretary for Technology

National Institute of Standards and Technology

Raymond G. Kammer, Director 
National Institute of Standards and Technology

Special Publication 941

Natl. Inst. Stand. Technol.

Spec. Publ. 941

92 pages (July 1999)

CODEN: NSPUE2
U.S. Government Printing Office

Washington: 1999

For sale by the Superintendent of Documents

U.S. Government Printing Office Washington, DC 20402 


\section{Foreword}

The Office of Standards Services periodically develops and publishes standards-related documents as a service to producers and users of standards and conformity assessment programs, both in government and in the private sector. This report is complementary to NIST Special Publication 912, Profiles of National Standards-Related Activities, and is designed to provide the reader with additional information about the conformity assessment infrastructure of the countries in the Western Hemisphere that will make up the Free Trade Area of the Americas (FTAA). We hope that this material is informative, helps businesses make appropriate contacts and will serve to stimulate a wider understanding of conformity assessment and trade in the Americas. 


\section{Acknowledgments}

I would like to thank NIST standards attachés Ileana Martinez in Brasil and Ivan Rios in México, NIST colleagues Henry Oppermann, Sharrill Dittmann, Mary Saunders, Libby Parker and Walter Leight for their timely inputs, updates and personal support. Thanks also to Aubrey Ponce and Karen Lusk for their invaluable computer support.

I would also like to thank all the participants of the NIST Standards in Trade Workshops for the Americas, who provided and verified information on the conformity assessment activities of their corresponding organizations and countries. Their time and effort are greatly appreciated. I would like to thank all the friends and colleagues I have met and made while gathering the information for this publication. 


\begin{abstract}
This publication summarizes the status of the technical infrastructure for conformity assessment in the Americas. It provides information about inspection and testing, product certification, quality system registration and laboratory accreditation for the countries that will comprise the Free Trade Area of the Americas.

Key words: Conformity assessment, Free Trade Area of the Americas (FTAA), laboratory accreditation, metrology, product certification, quality system registration, standards, Technical Barriers to Trade Agreement (TBT), World Trade Organization (WTO).
\end{abstract}




\section{Table of Contents}

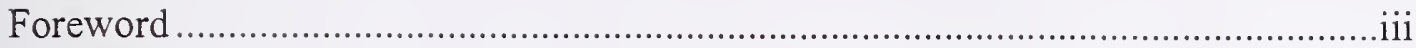

Acknowledgments................................................................................

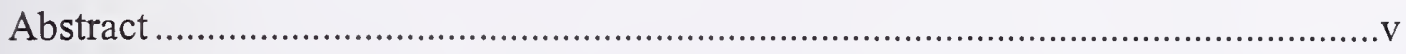

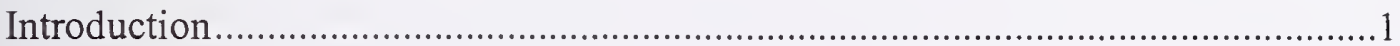

Section I: FTAA Countries Conformity Assessment Infrastructure......................4

Section II: Membership in International and Regional Bodies............................39

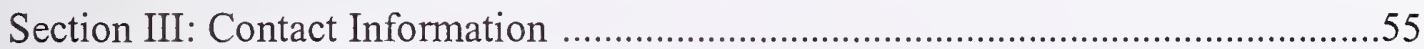

Useful Web Sites................................................................................... 70

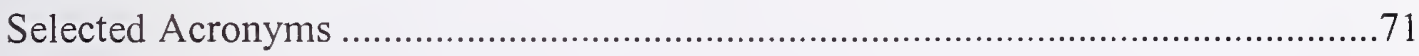

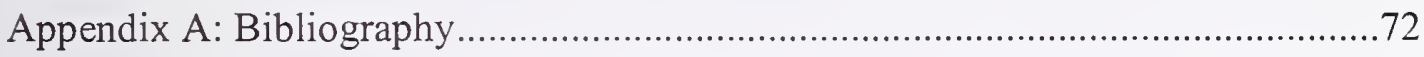

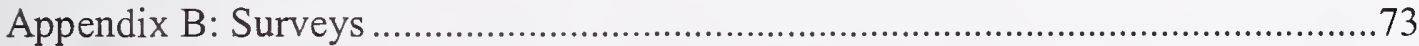





\section{Introduction}

In December 1994, at the First Summit of the Americas held in Miami, the leaders of thirty-four Western Hemisphere countries met to launch the Free Trade Area of the Americas (FTAA) and to commit to its establishment by the year 2005 . The initiative proposed to eliminate barriers to trade in goods and services for the region and to promote greater prosperity through economic integration and free trade. The FTAA will be the largest market in the world, with more than 700 million people (14 percent of the world population) and a combined Gross Domestic Product (GDP) of nearly $\$ 7$ trillion, or approximately 31 percent of the global wealth.

The FTAA trade ministers have recognized the importance of standards, metrology and conformity assessment procedures for facilitating international trade. Furthermore, the FTAA negotiators understand that to achieve a meaningful free trade area, products and services must be traded in the region without the burden of duplicative testing requirements, certifications and other prerequisites that do not address legitimate regulatory concerns, consumer needs or market demands.

The Americas are made up of a great variety of countries at different stages of technical development, resulting in a wide variety of conformity assessment infrastructures. Some countries have a long history of standardization and conformity assessment. Others are newer at this task and look to their neighbors to form regional or bilateral agreements that will satisfy their conformity assessment requirements without necessarily setting up an expensive and complex national system of their own. Still others are setting up parts of the conformity assessment system commensurate with the realities of their economies, social infrastructure and the globalization of markets.

Many sources were consulted to gather and verify the information in this publication, they are listed in the bibliography. The challenges in compiling the information were (a) to define clearly for the various infrastructures the information being sought; (b) to determine where to get the data, and (c) to verify the information obtained. Even though 33 FTAA countries are signatories to the World Trade Organization Technical Barriers to Trade Agreement (WTO TBT), which requires the establishment of an enquiry point, countries differ in their interpretation of basic WTO obligations and ways to comply with these obligations. Many of the FTAA countries do not yet have an operational enquiry point, which is the logical place to obtain information on standards and conformity assessment. In some cases, it was very difficult to locate the appropriate information, and for two countries, namely, St. Kitts and Nevis and St. Vincent and the Grenadines, very little information was obtained at all.

\section{Conformity Assessment}

Conformity assessment ${ }^{1}$ is a comprehensive term for measures taken by manufacturers, their customers, regulatory authorities, and independent third parties to assess conformity to relevant standards. Conformity assessment procedures ensure that products, processes and services meet certain characteristics in a consistent manner and include inspection, testing, certification, quality

\footnotetext{
'See "Standards, Conformity Assessment and Trade into the 21st century", National Research Council, National Academy Press, Washington, 1995, pp. 65.
} 
and environmental management system registration, and accreditation activities. Four conformity assessment activities were chosen as the basis for comparison among the FTAA countries as listed in Section I. They are: (a) Inspection and Testing, (b) Product Certification, (c) Quality System Registration, and (d) Laboratory Accreditation. The categories follow the definitions established by the International Organization for Standardization/International Electrotechnical Commission (ISO/IEC) Guide 2, which defines:

Testing as the action of carrying out one or more technical operations or tests to determine the characteristics of a given product, process or service according to a specified technical procedure or test method.

Inspection as conformity evaluation by observation and judgment accompanied as appropriate by measurement, testing or gauging.

Certification as a procedure by which a third party gives written assurance that a product, process or service conforms to specified requirements.

Certification is distinguished from testing by three key features ${ }^{2}$ :

1. Certification always measures a product, process or service against one or more specific standards, whether mandatory or voluntary. Testing, by contrast, does not necessarily measure against any specific standard;

2. Certification is always performed by a third party, independent of either the supplier or the purchaser;

3. Certification results in a formal statement of conformity, a certificate that can be used by the manufacturer to show compliance with regulations, meet purchasing specifications, and enhance the product's marketability.

Product Certification as the process of providing assurance that a product conforms to a standard or specification. It encompasses many different levels of complexity and expense, depending on the characteristics of the product and the degree of need for confidence in the product's conformity to standards.

Quality System Registration (sometimes referred to as "quality system certification") as the assessment and periodic audit of the adequacy of a supplier's quality management system by a third party, known as a registrar, against quality management standards such as the ISO 9000 series. Quality system registration does not imply product quality or conformity to any given set of requirements. It only guarantees that a product is made consistently to a certain quality level set by the manufacturer.

Laboratory Accreditation as the process that determines whether a laboratory is capable of performing specified test methods and procedures correctly to the level of accuracy' required for a given specification. It does not ensure that the laboratory competently and consistently conducts the tests. Furthermore, laboratory accreditation provides assurance about the capability of a laboratory only within the scope or areas for which accreditation was granted.

\footnotetext{
${ }^{2}$ Ibid. pp 73 .
} 


\section{Content}

The information in this report is divided into three main sections. Section I is a brief description for each FTAA country with respect to the four headings discussed above. Section II is a series of tables listing membership by FTAA countries in standards, metrology, conformity assessment bodies and regional trade agreements. It is important to read the explanatory notes that accompany each table as the notes define column headings and specify whether membership is by country or by individual conformity assessment bodies. It is also important to read a country's individual description to complement the information summarized in the tables. Sections I and II should be consulted together. Section III lists a series of contacts that allow the reader to obtain further information about specific subjects. For all the sections the names of the countries follow the Organization of American States convention, that is, each country is listed by its name in its official language.

\section{Future Updates}

Despite the efforts to be comprehensive, this publication will need regular updates that reflect not only the dynamic changes taking place in the FTAA countries but also many of the developments occurring at the international standards, metrology and conformity assessment level. Organizations or experts that feel that certain information has been overlooked or incorrectly reported are encouraged to send pertinent corrections or updates to:

Dr. Carmiña Londoño

National Institute of Standards and Technology

100 Bureau Drive, Stop 2100

Gaithersburg, MD 20899-2100

e-mail: carmina.londono@NIST.gov

Internet Website:http//ts.NIST.gov.oss

If it is more practical, information can be submitted using the surveys used for Section I and shown in appendix A. 


\begin{tabular}{|c|c|c|c|}
\hline \multirow{5}{*}{ 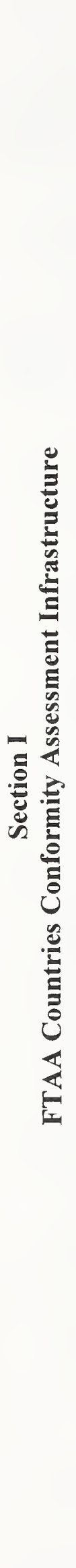 } & 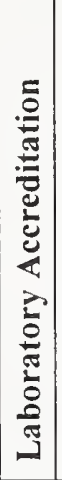 & 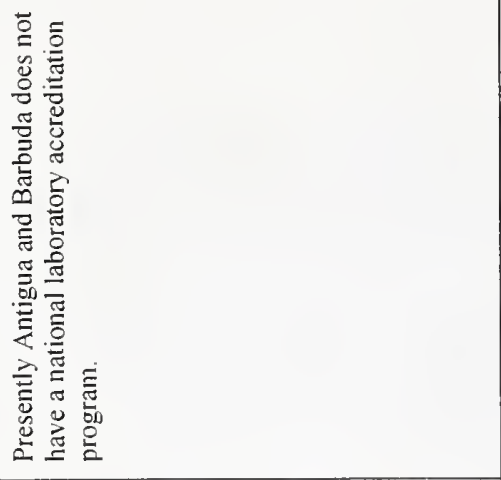 & 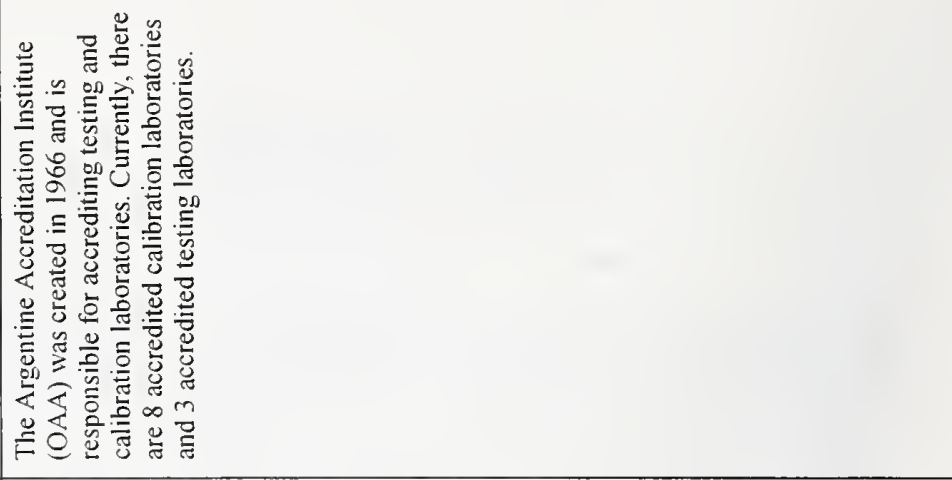 \\
\hline & 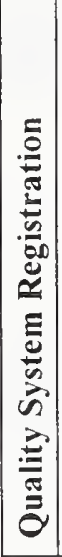 & 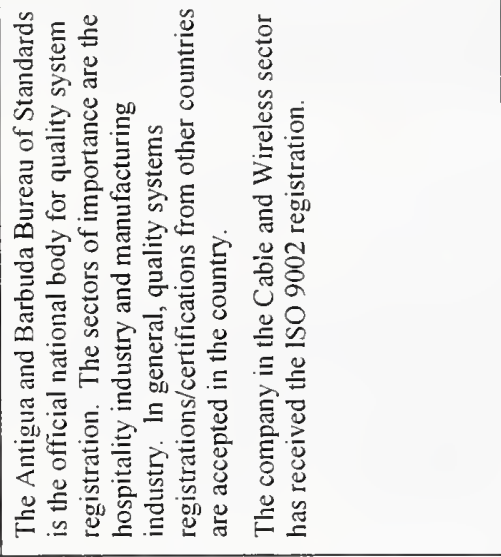 & 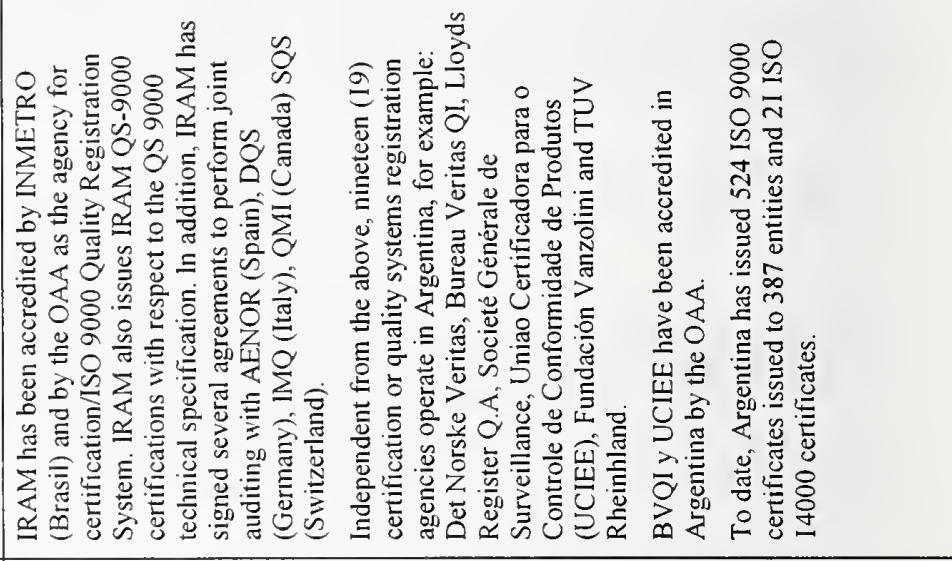 \\
\hline & 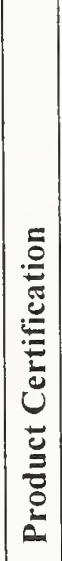 & 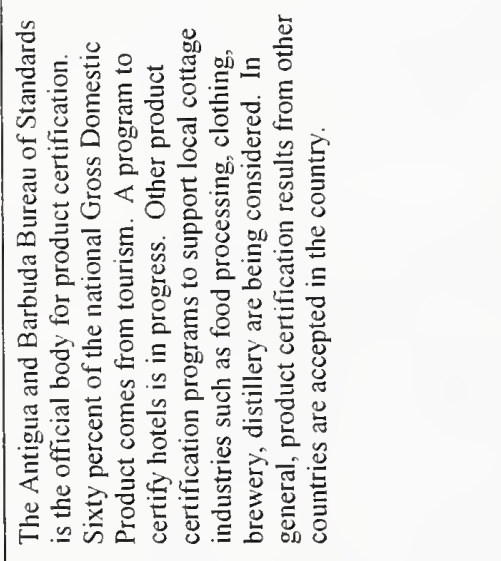 & 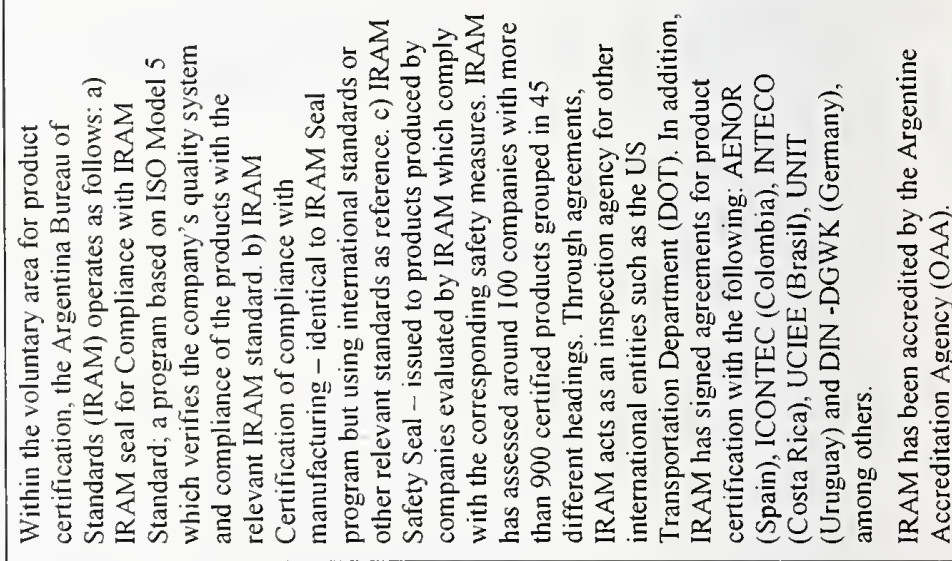 \\
\hline & 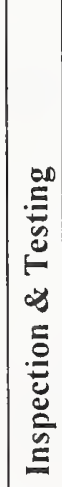 & 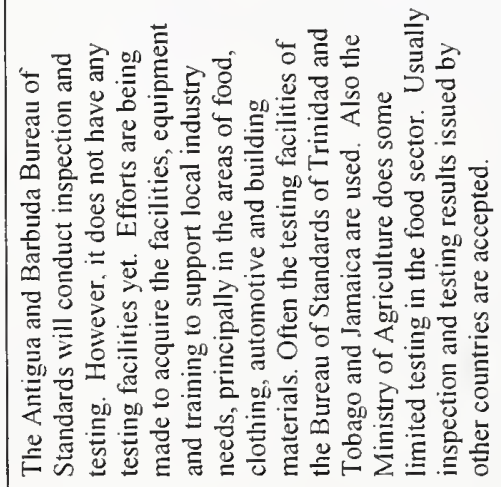 & 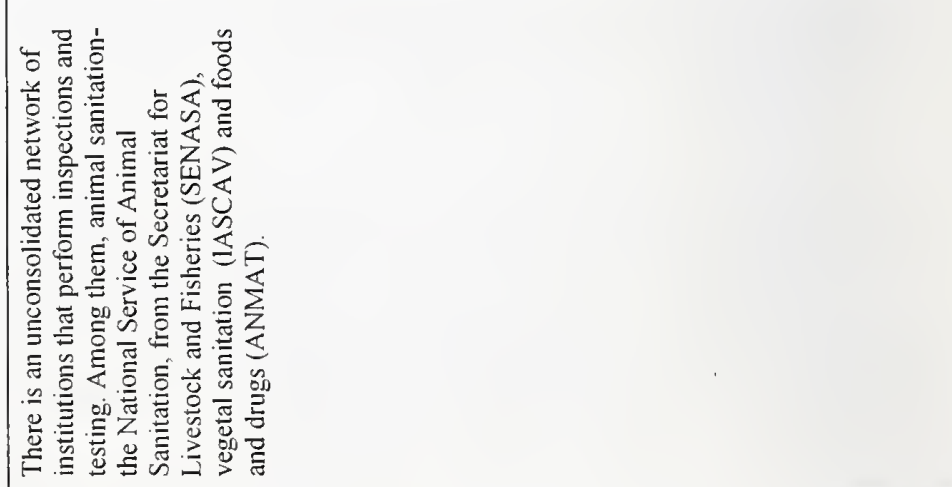 \\
\hline & 总 & 总 & 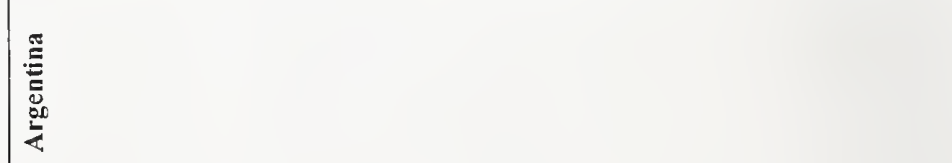 \\
\hline
\end{tabular}




\begin{tabular}{|c|c|c|}
\hline 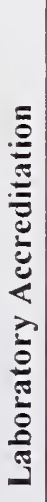 & 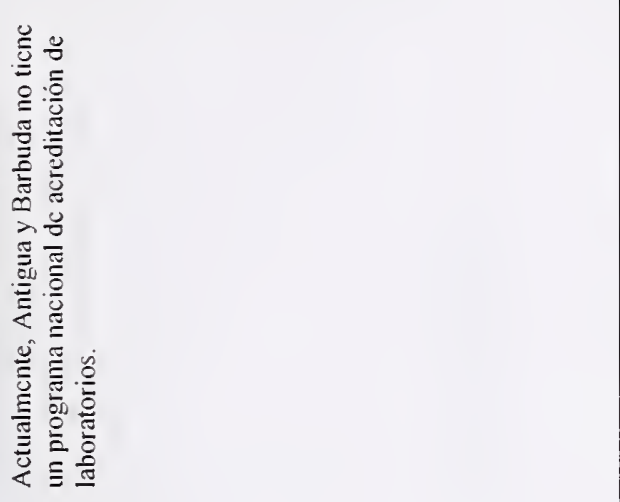 & 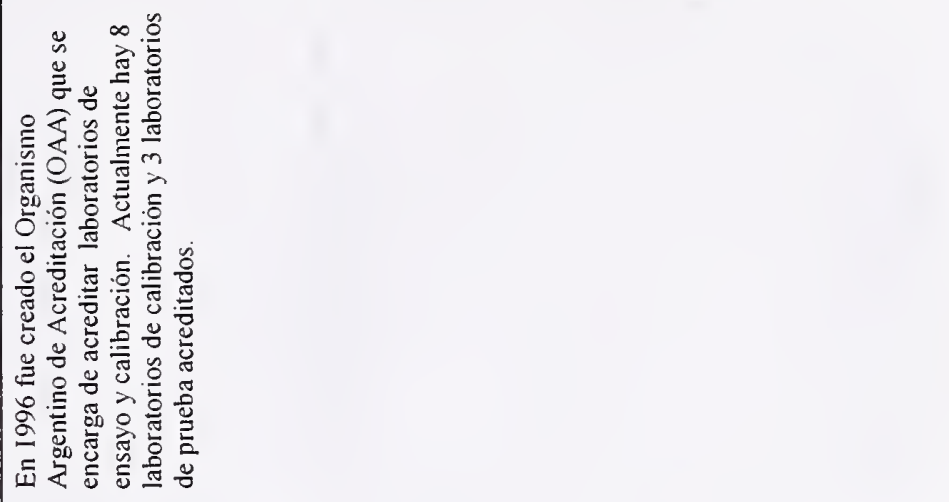 \\
\hline 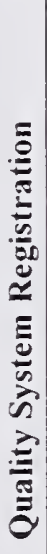 & 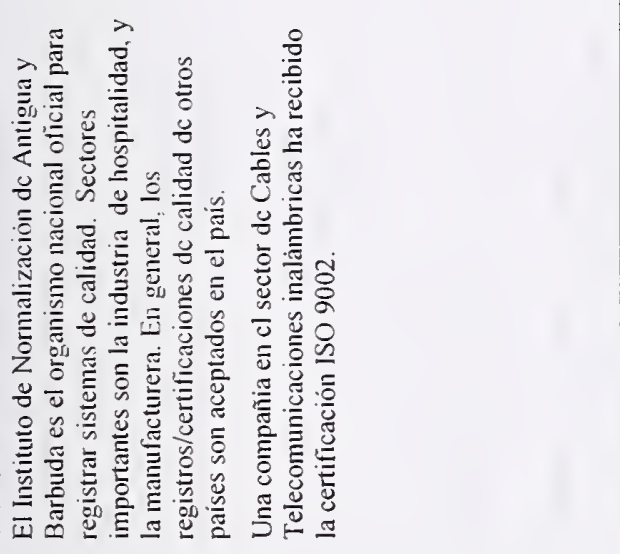 & 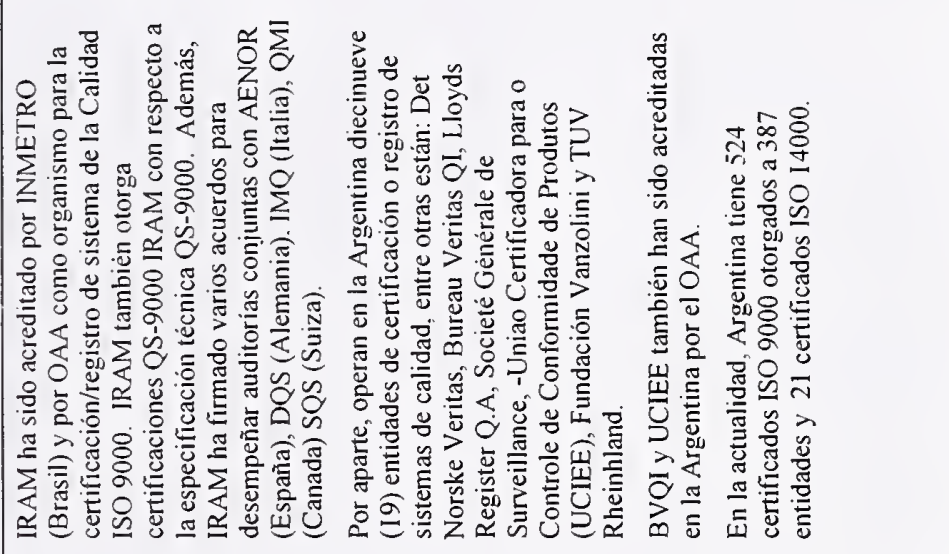 \\
\hline 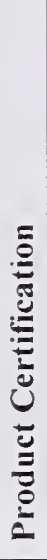 & 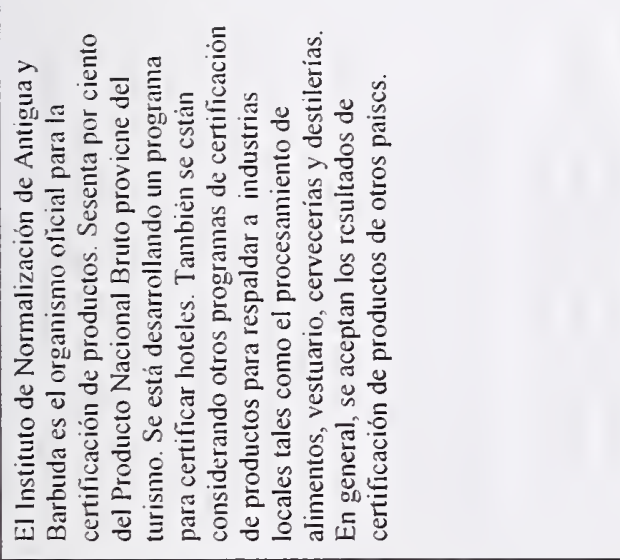 & 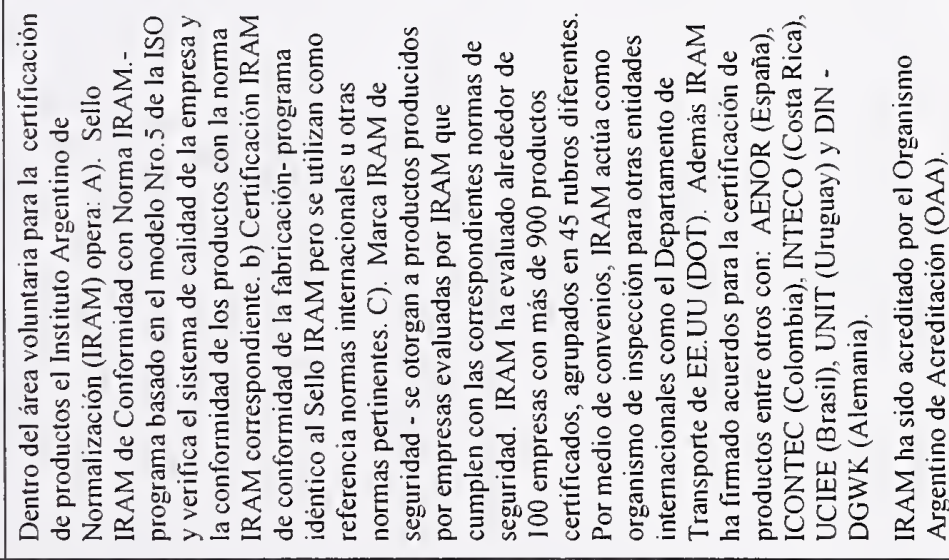 \\
\hline 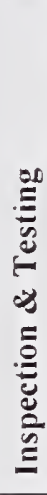 & 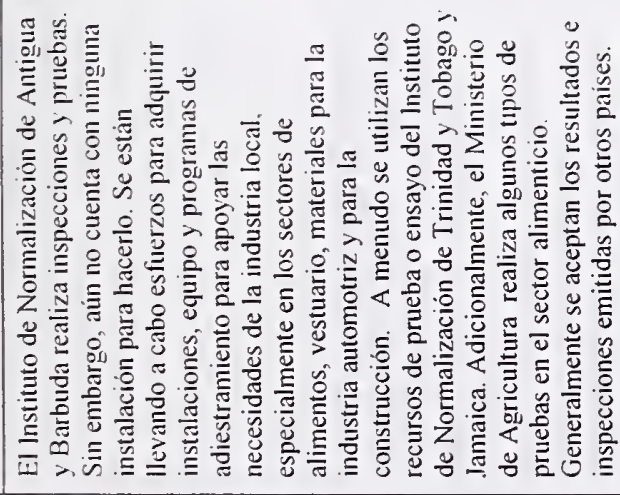 & 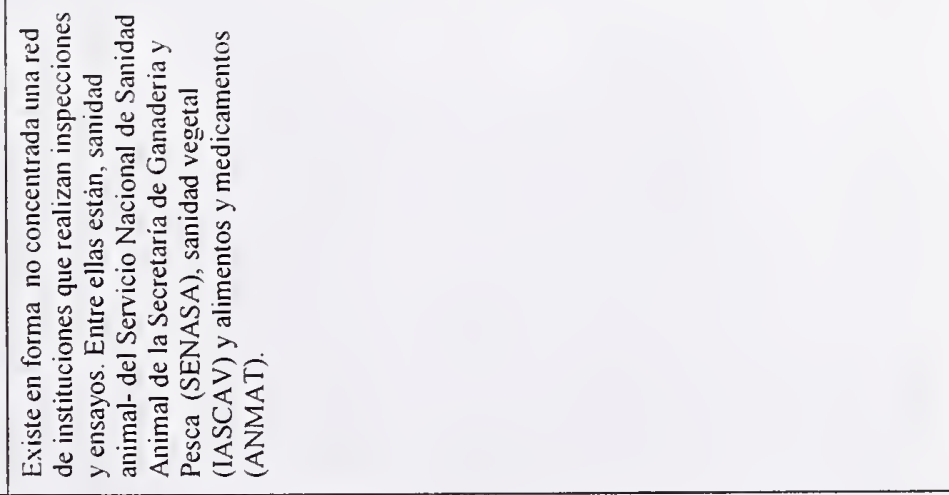 \\
\hline 咅 & 莒 & \\
\hline
\end{tabular}




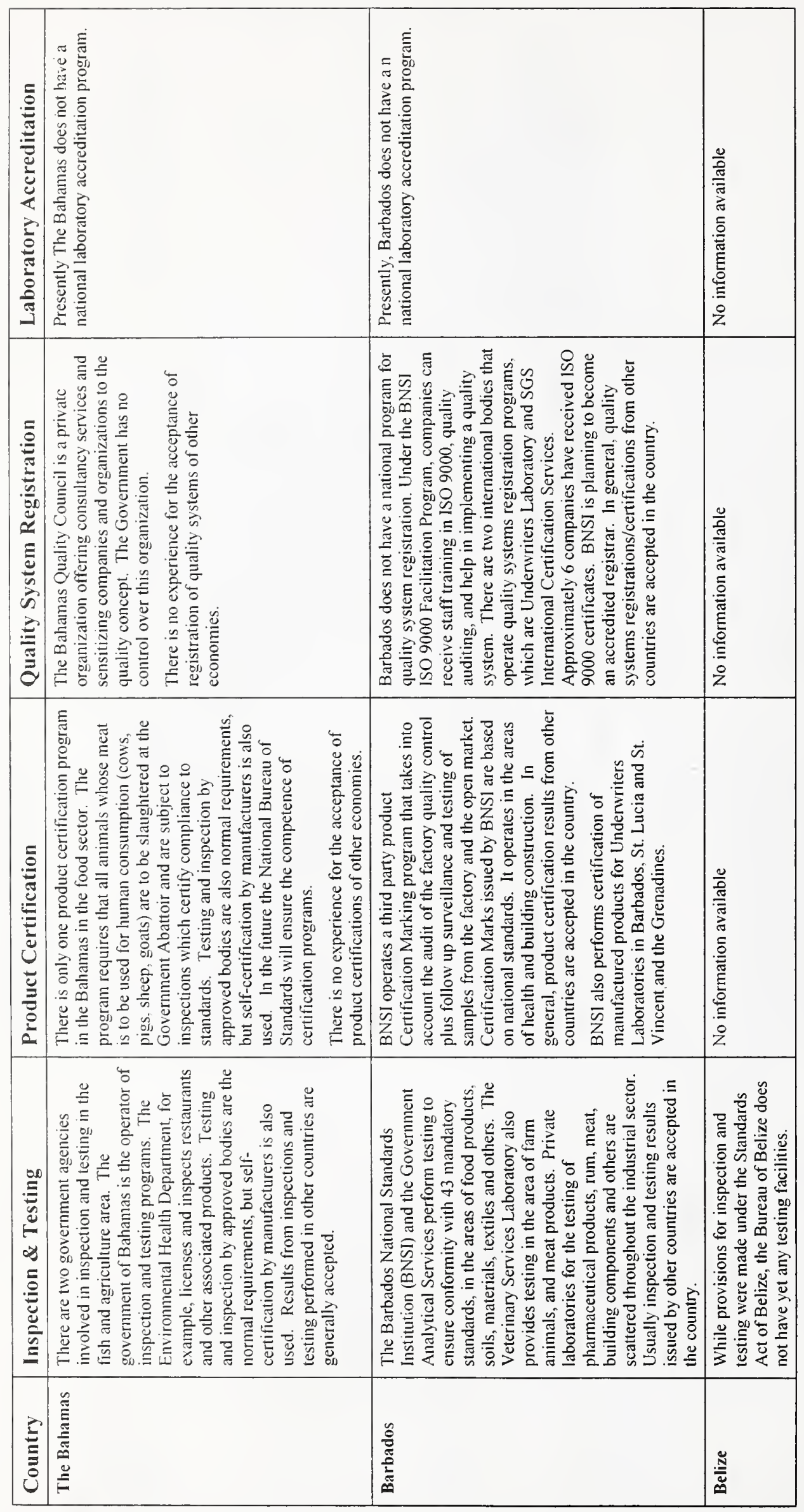




\begin{tabular}{|c|c|c|c|}
\hline 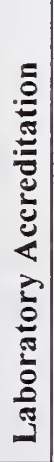 & 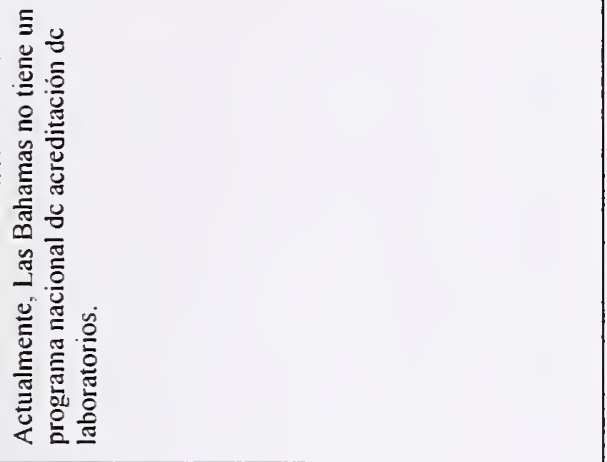 & 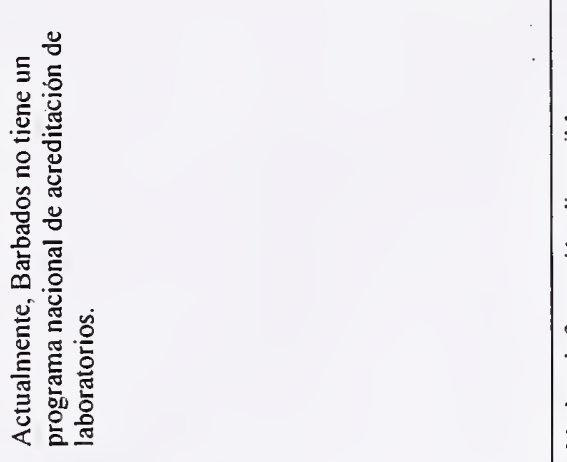 & 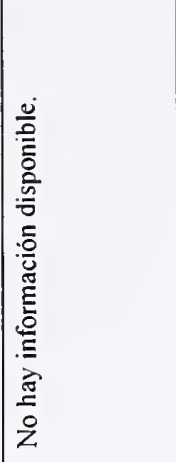 \\
\hline 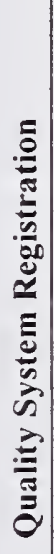 & 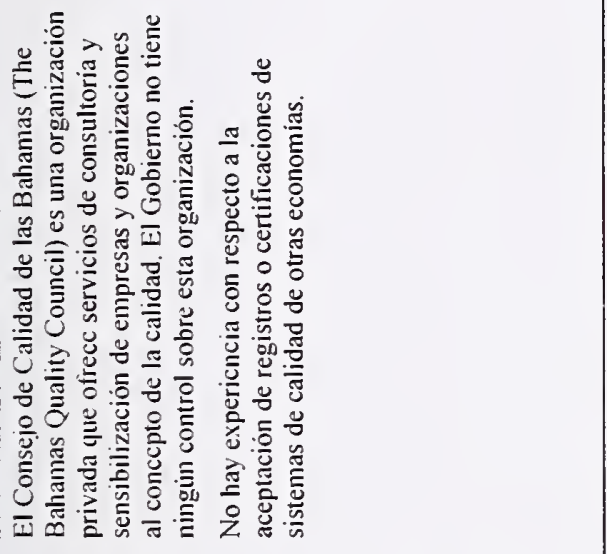 & 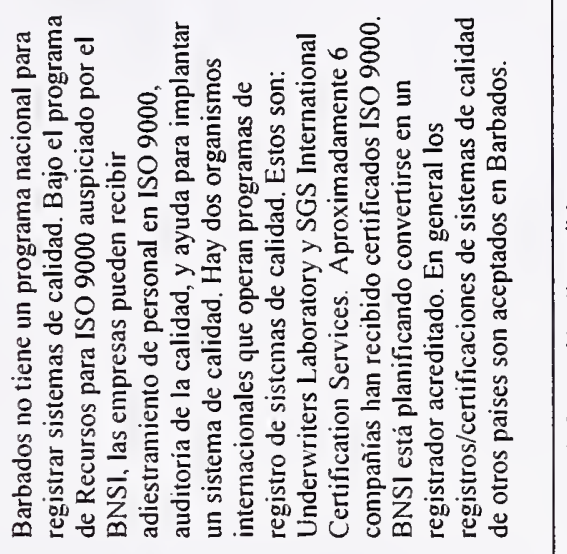 & 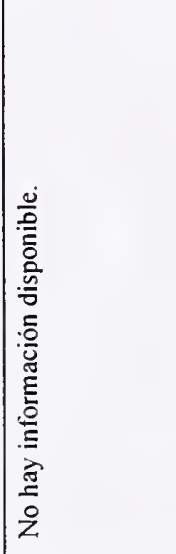 \\
\hline 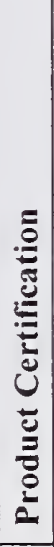 & 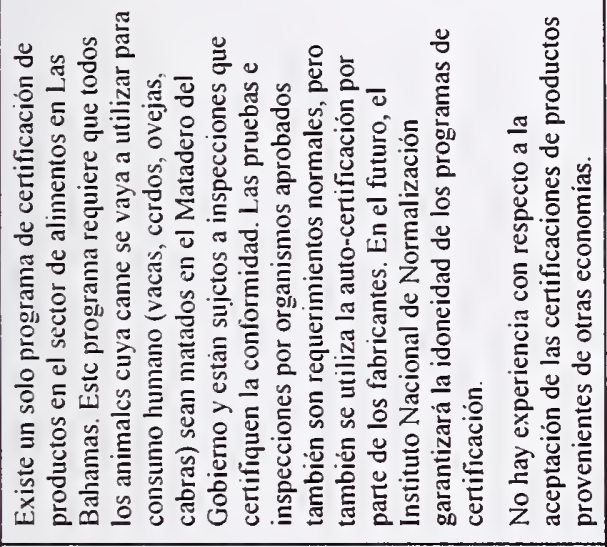 & 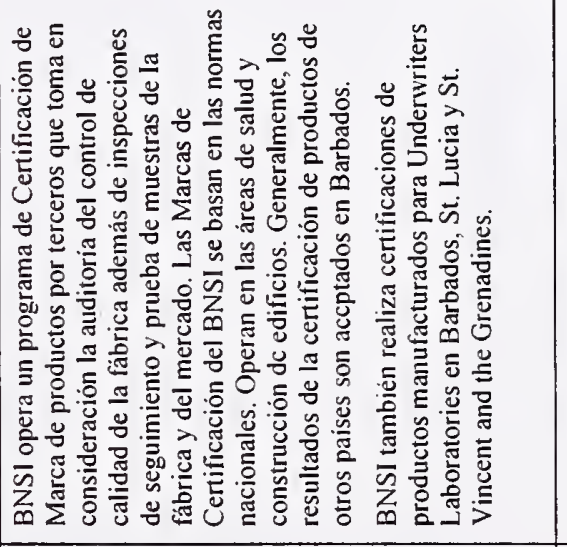 & 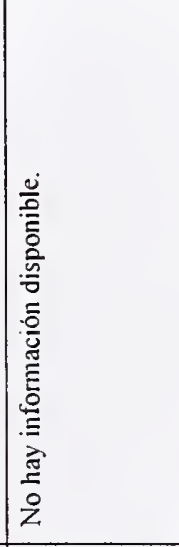 \\
\hline 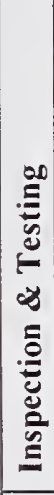 & 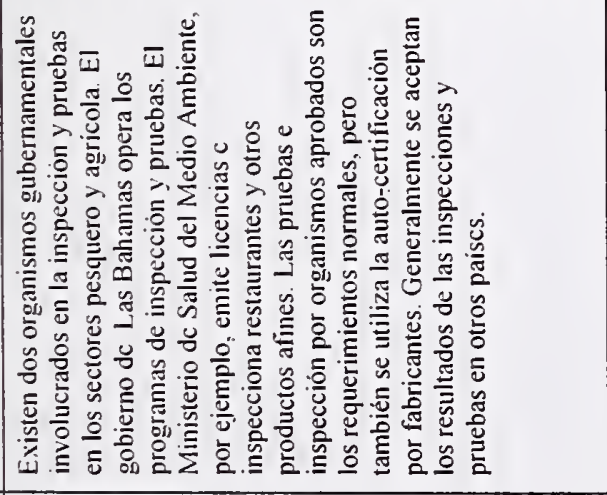 & 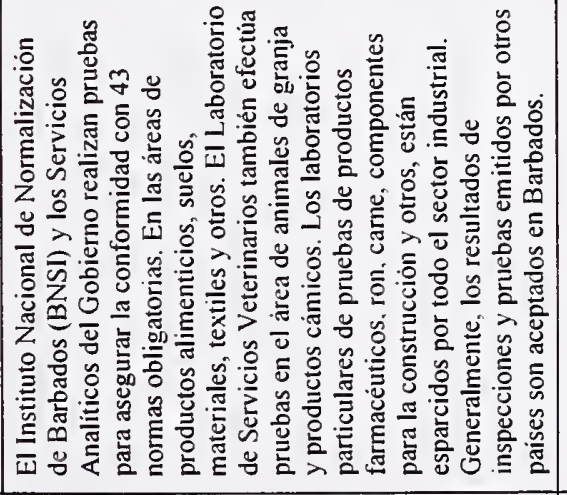 & 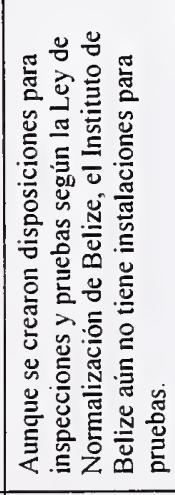 \\
\hline 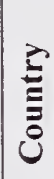 & 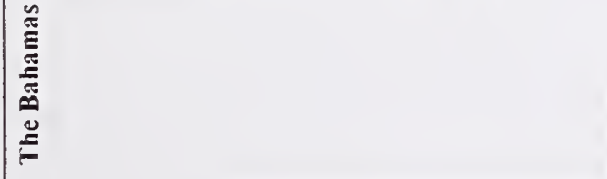 & 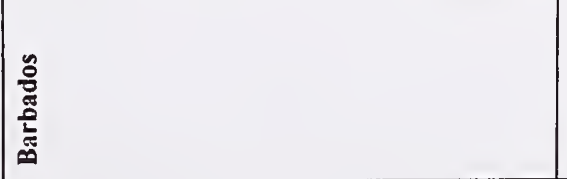 & 苂 \\
\hline
\end{tabular}




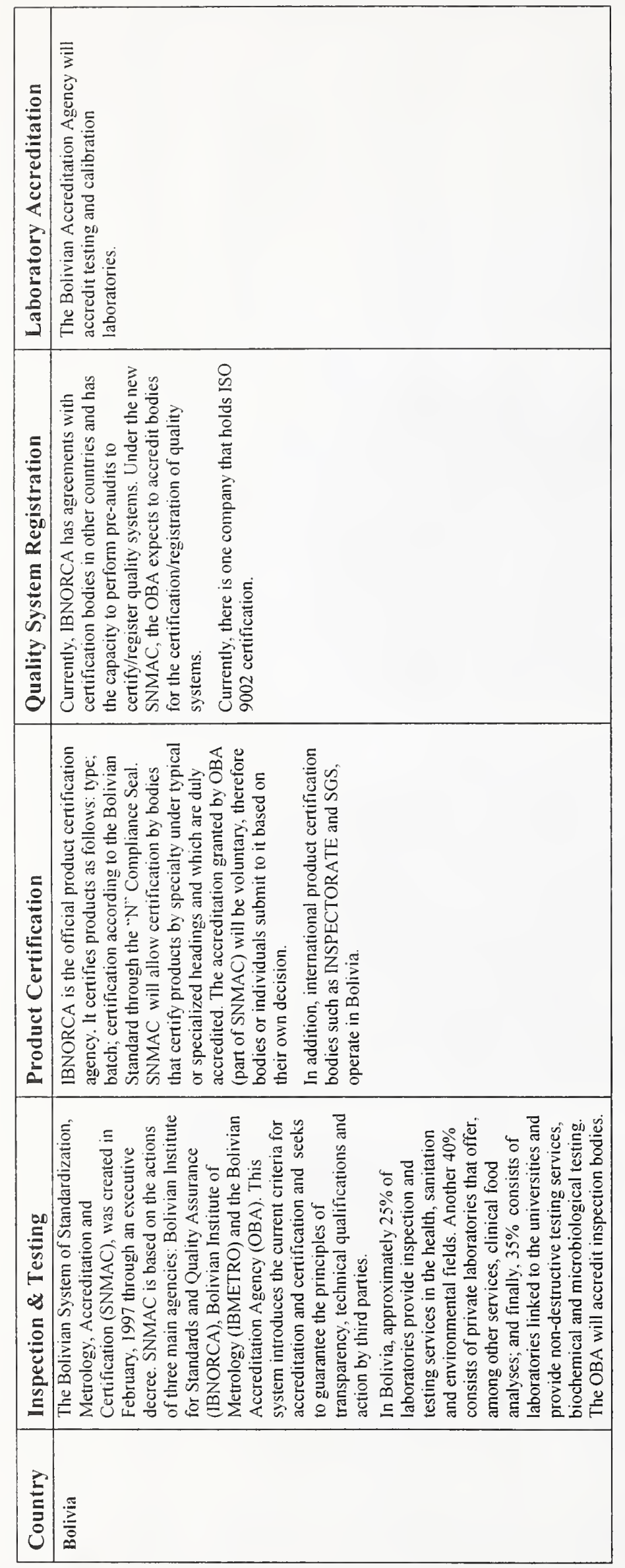



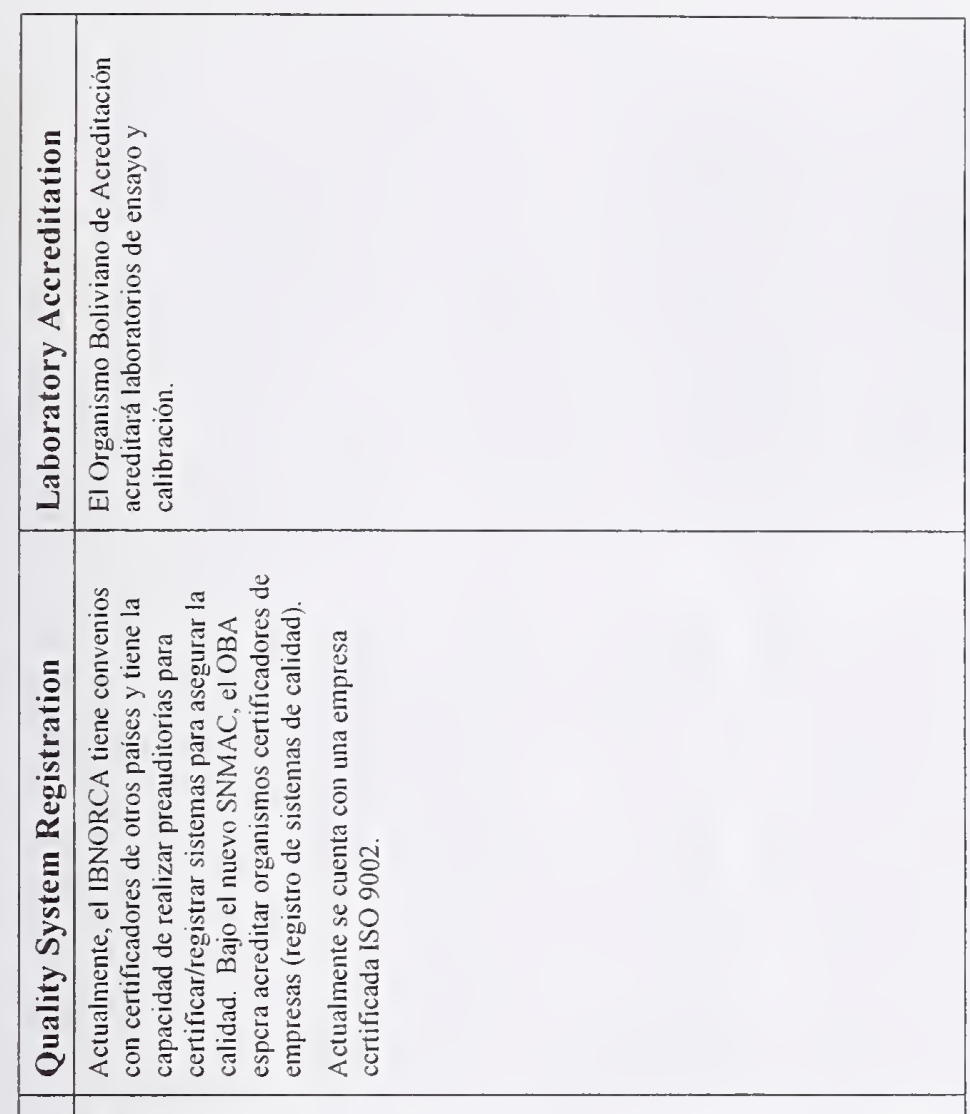

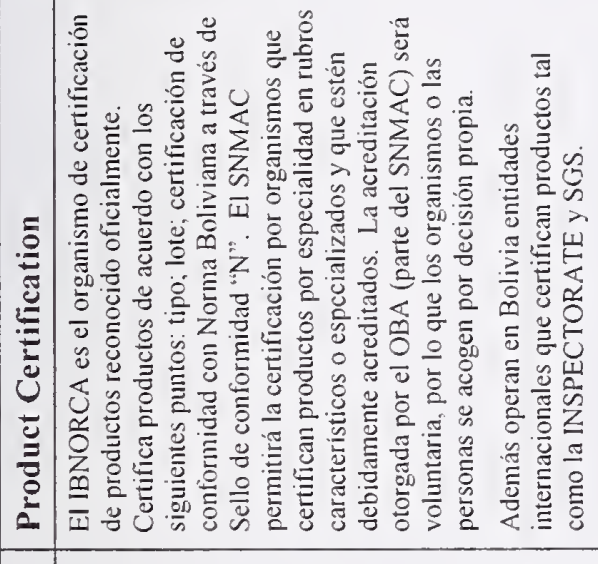

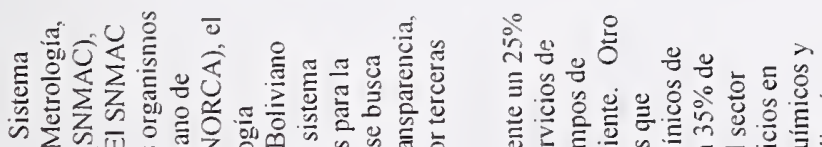

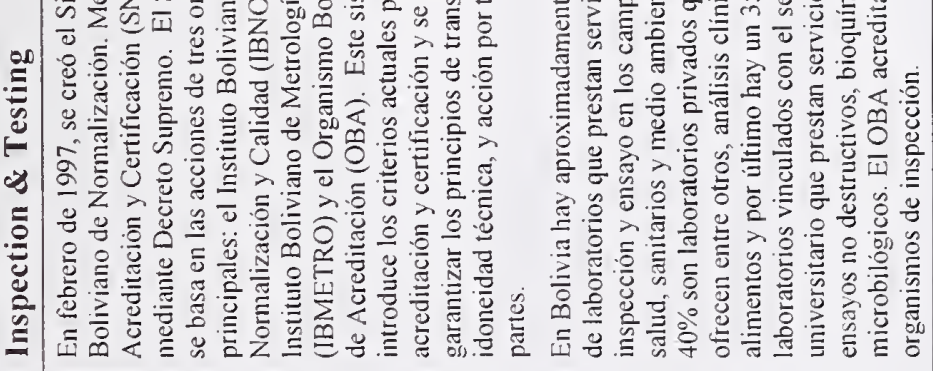

至 


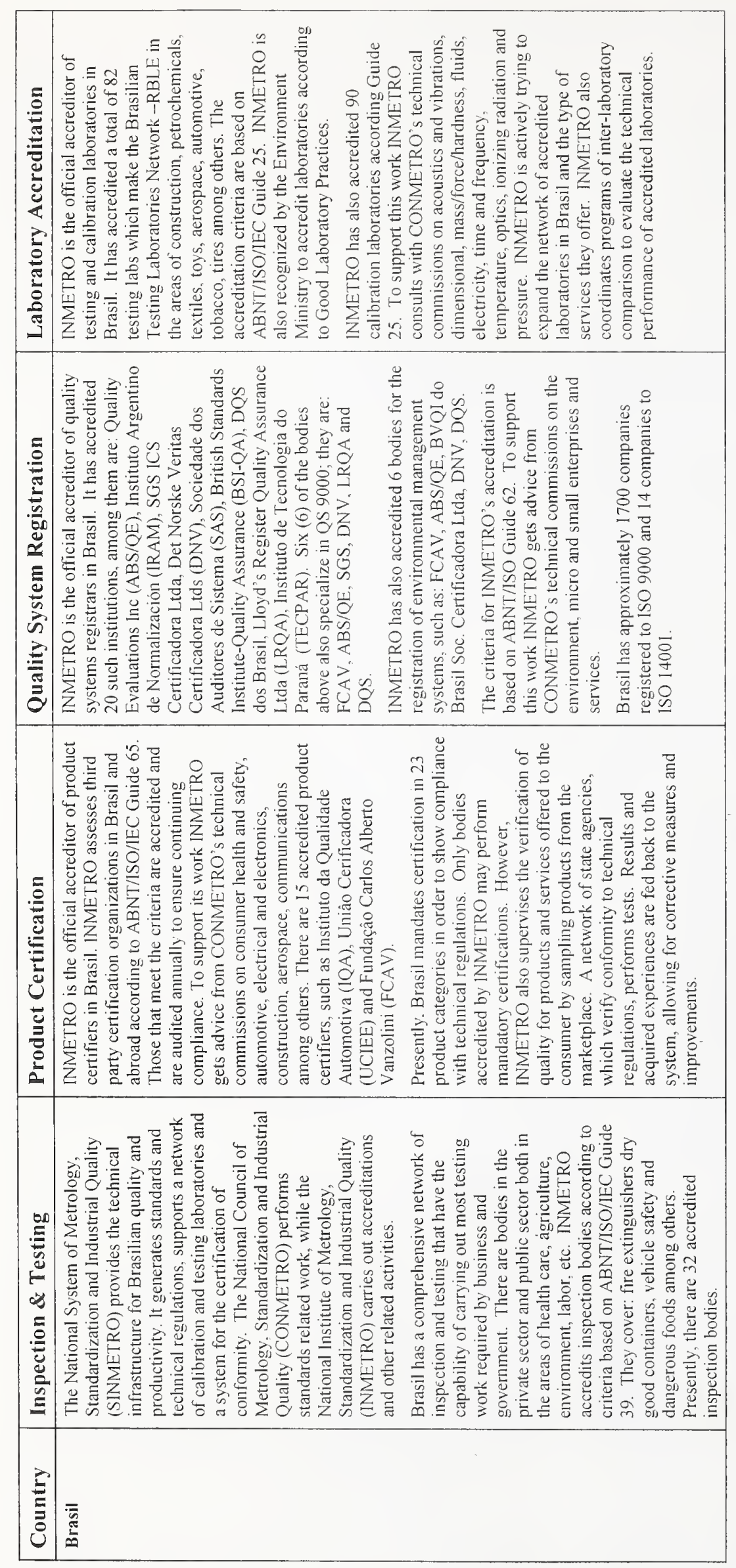




\begin{tabular}{|c|c|}
\hline & 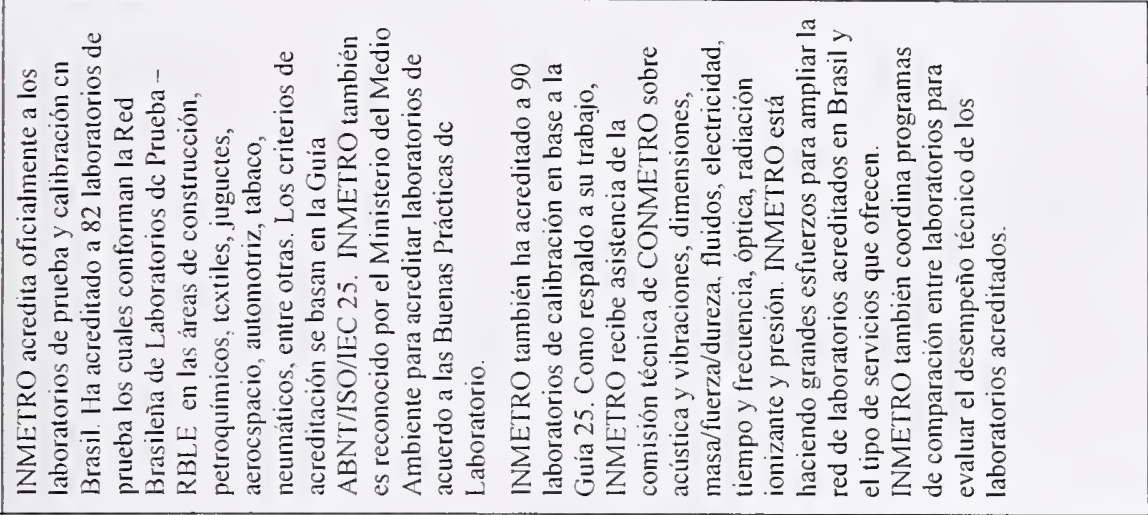 \\
\hline & 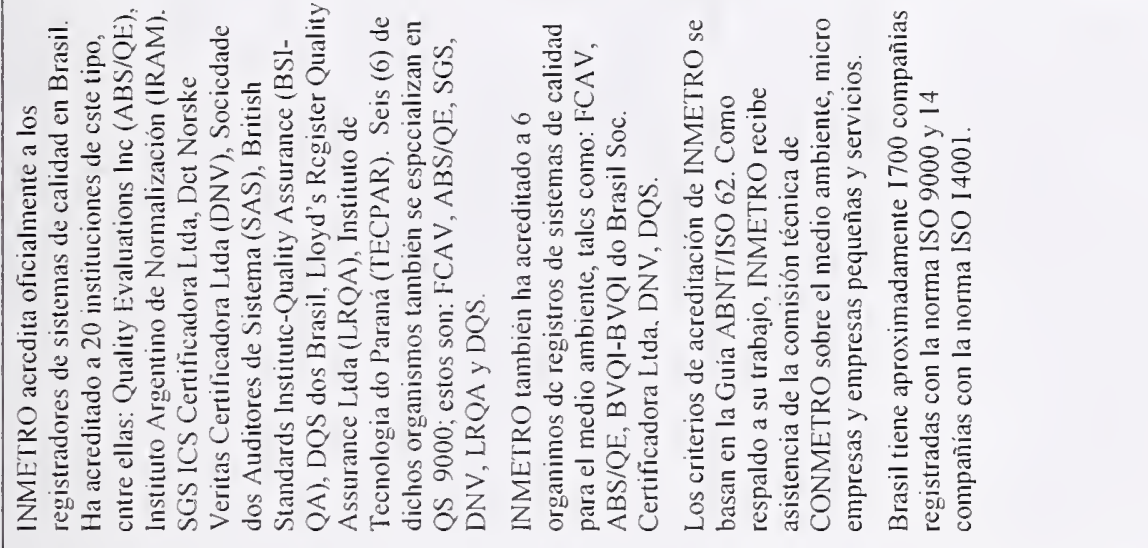 \\
\hline & 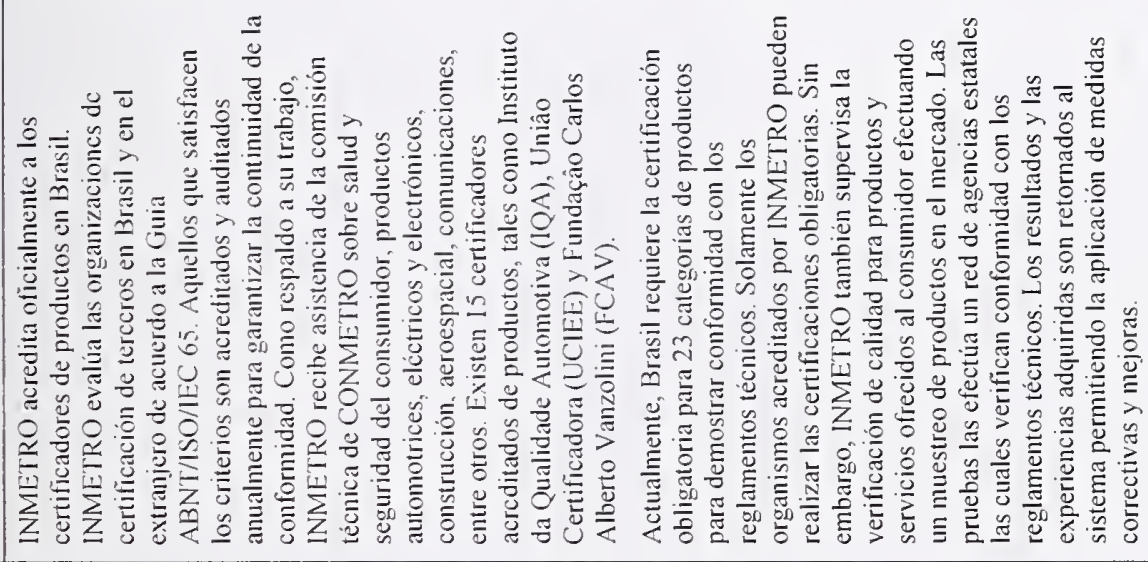 \\
\hline & 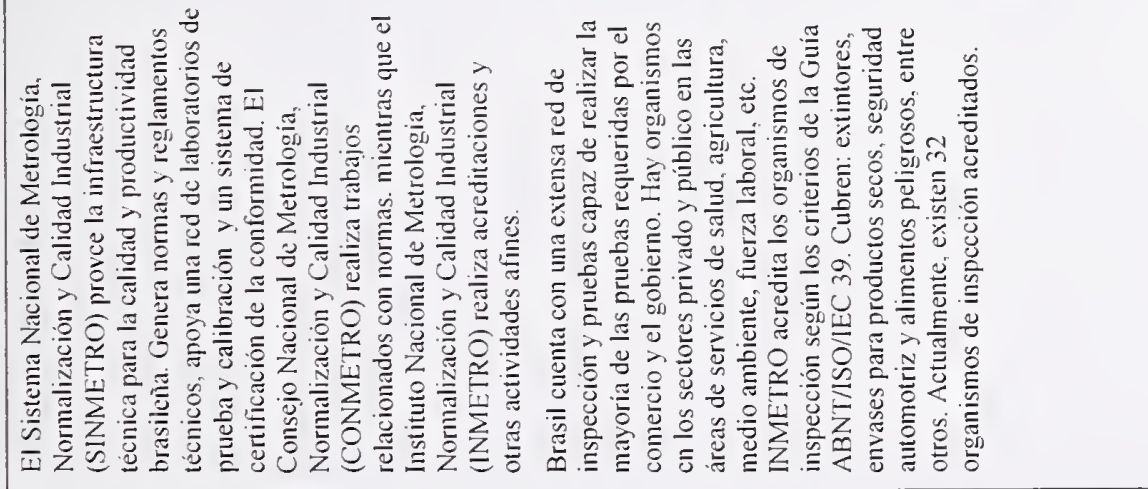 \\
\hline & \\
\hline
\end{tabular}




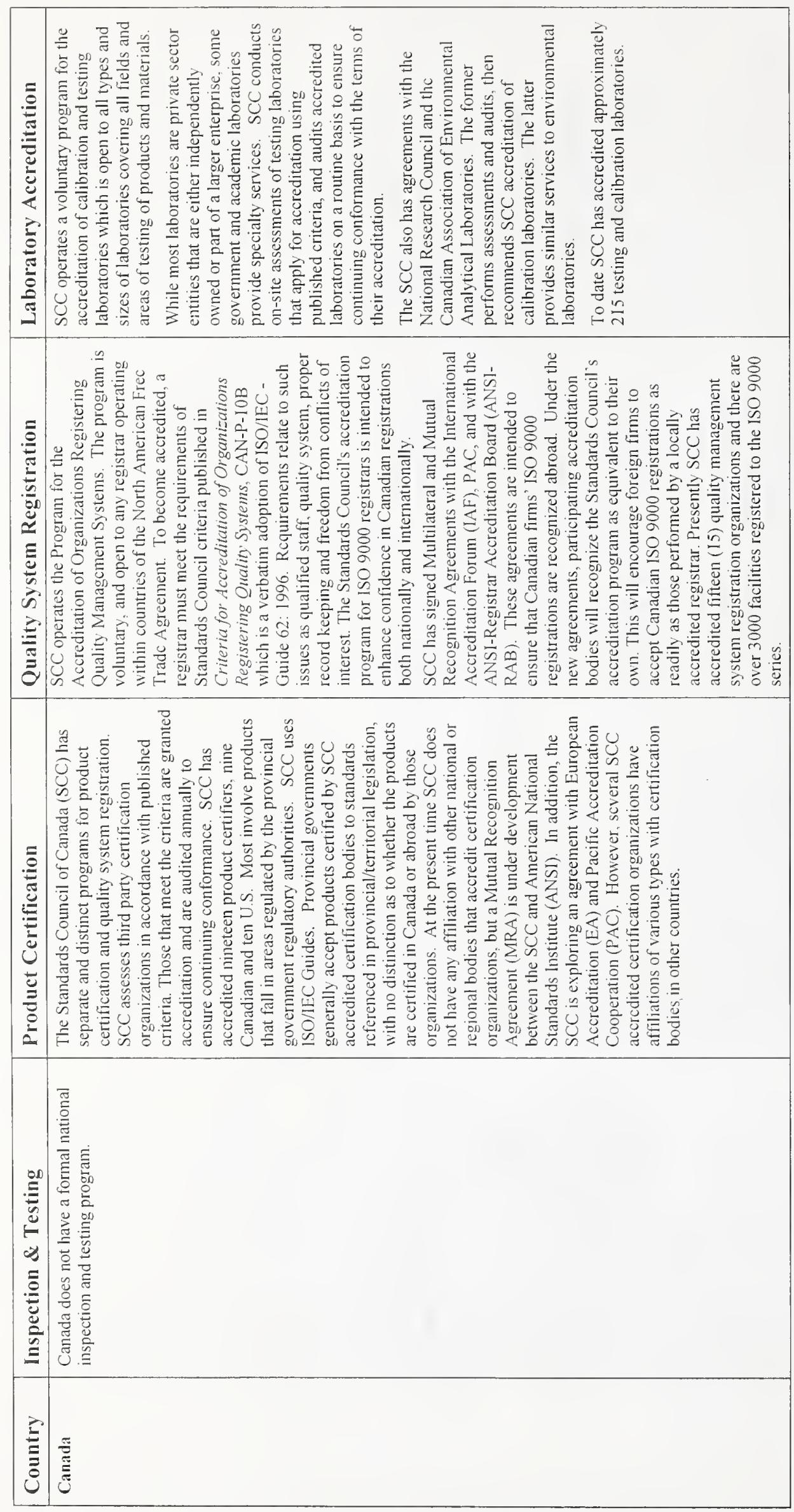




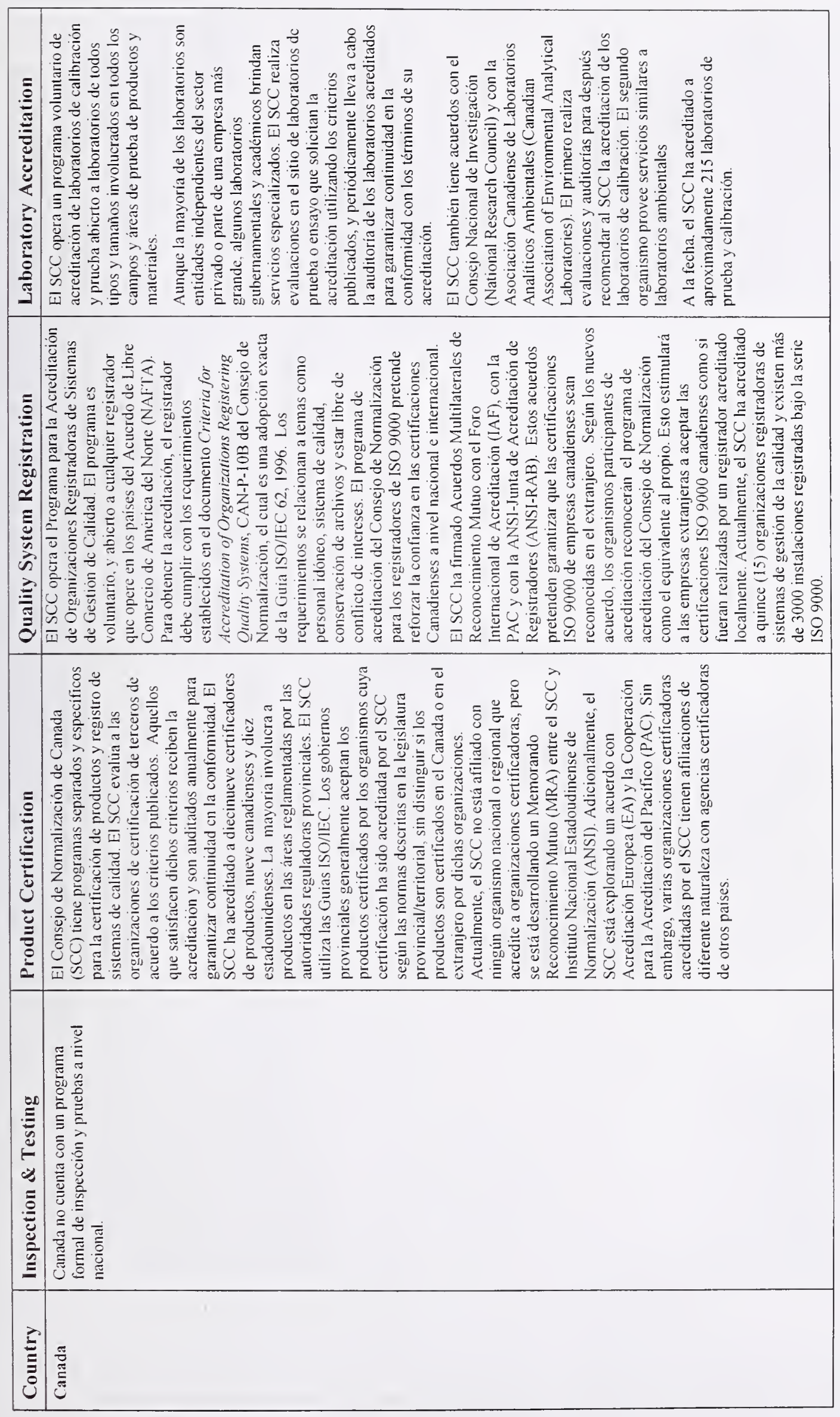




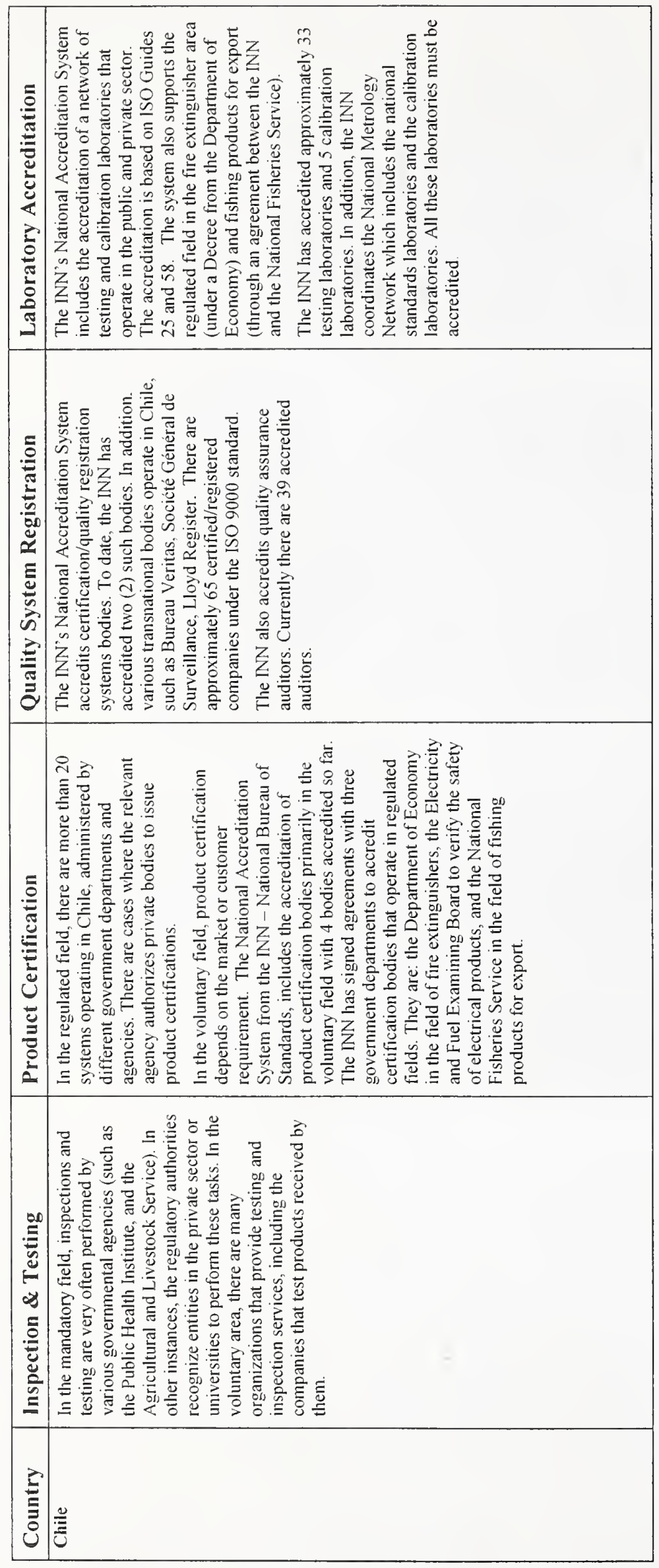




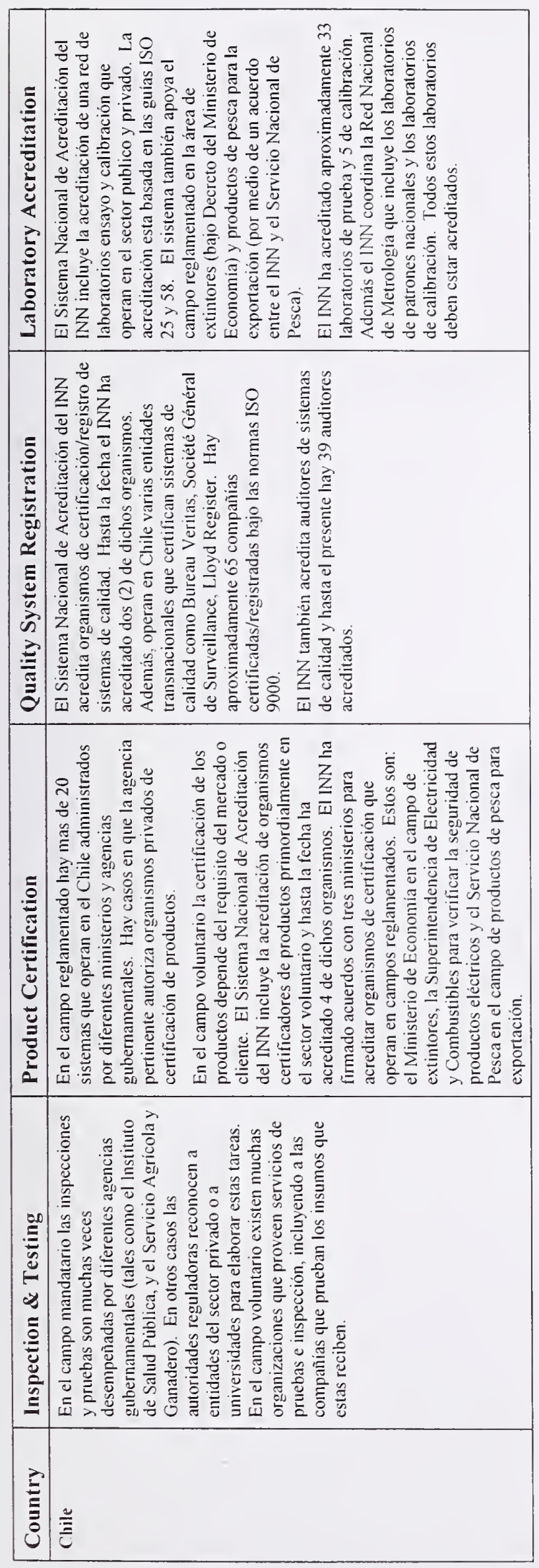




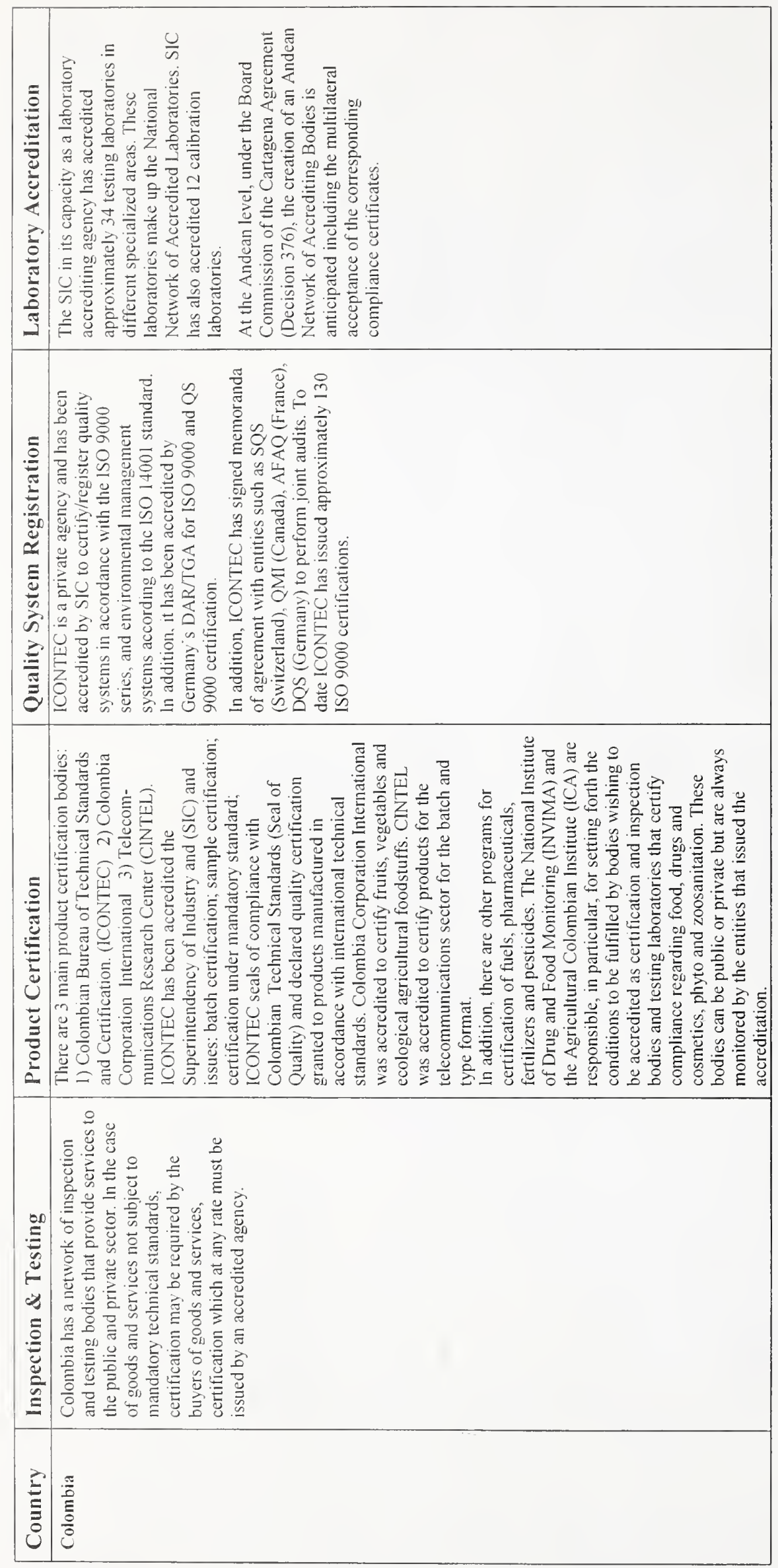




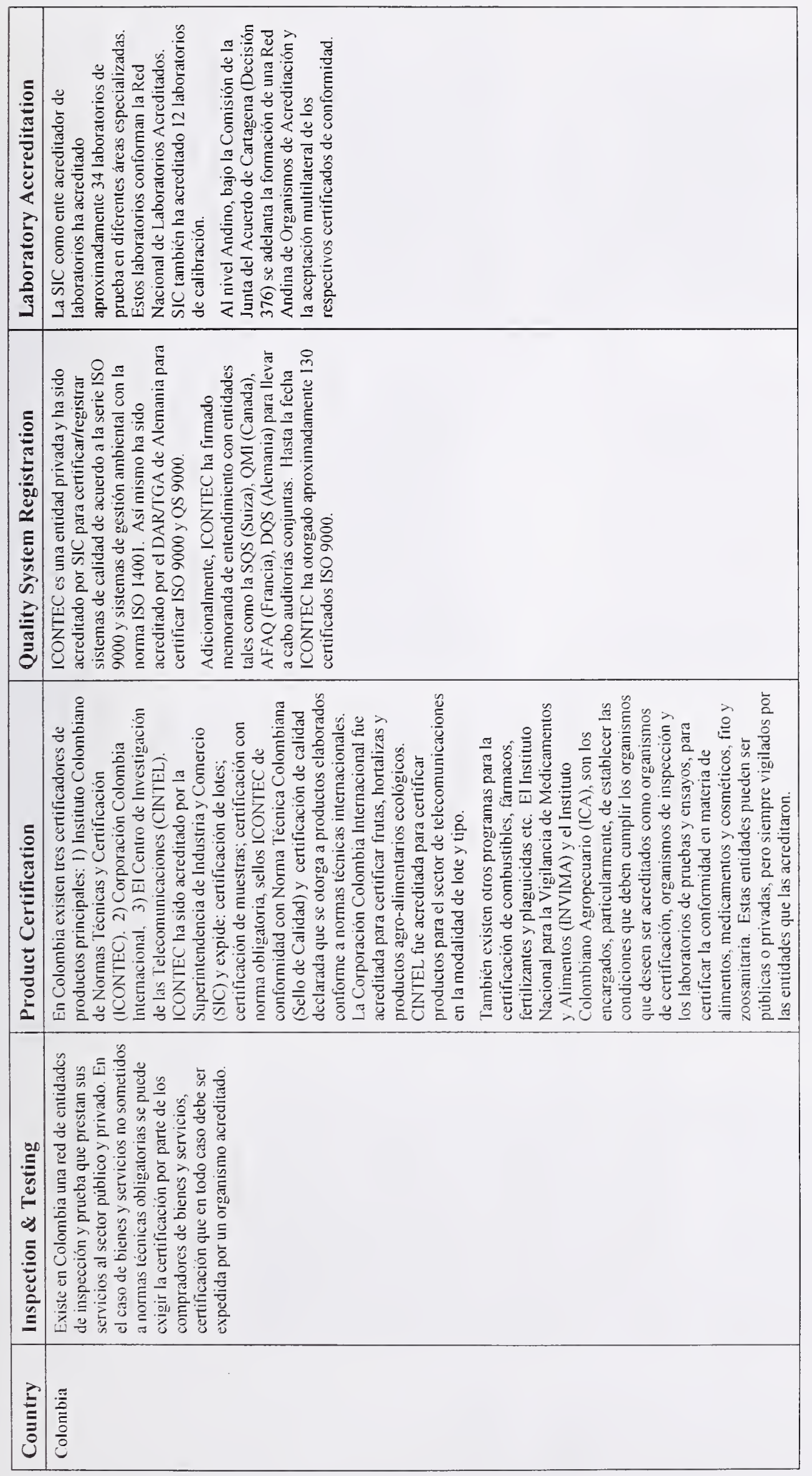




\begin{tabular}{|c|c|c|c|}
\hline 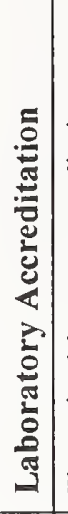 & 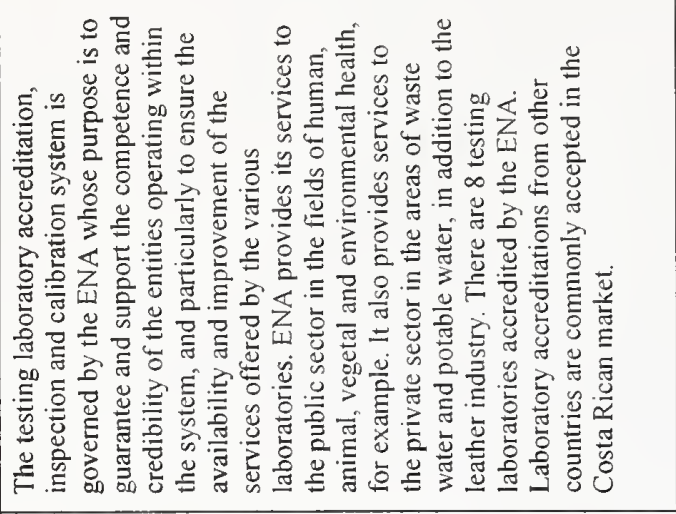 & 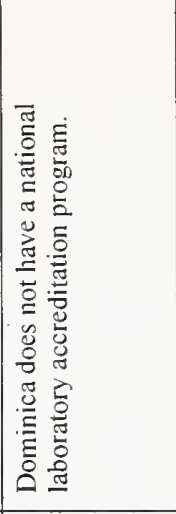 & 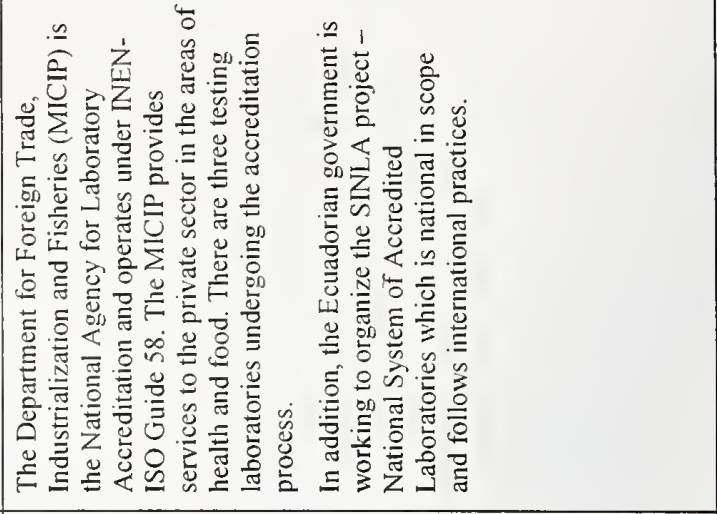 \\
\hline 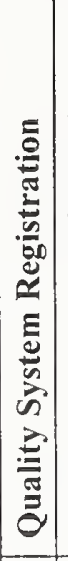 & 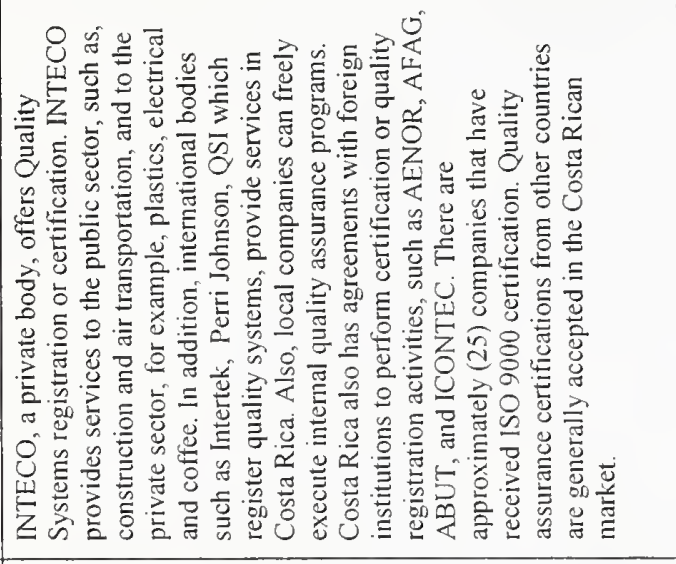 & 芒 & 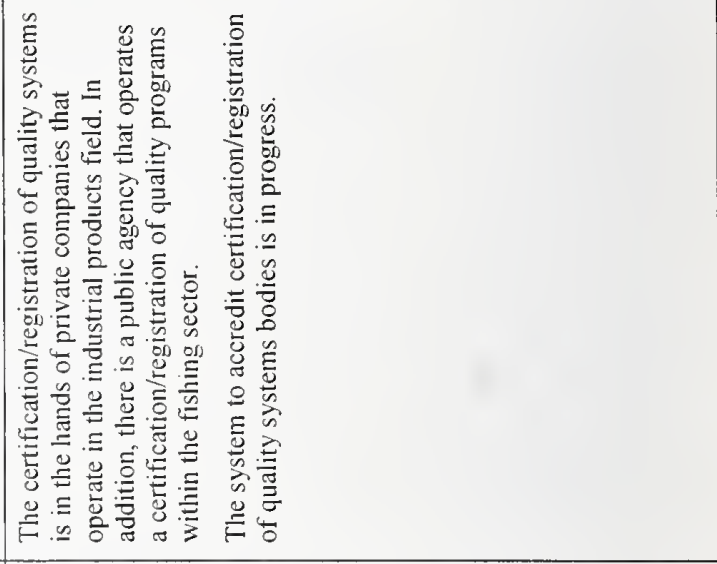 \\
\hline 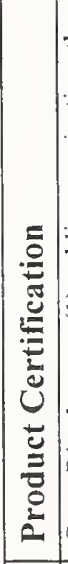 & 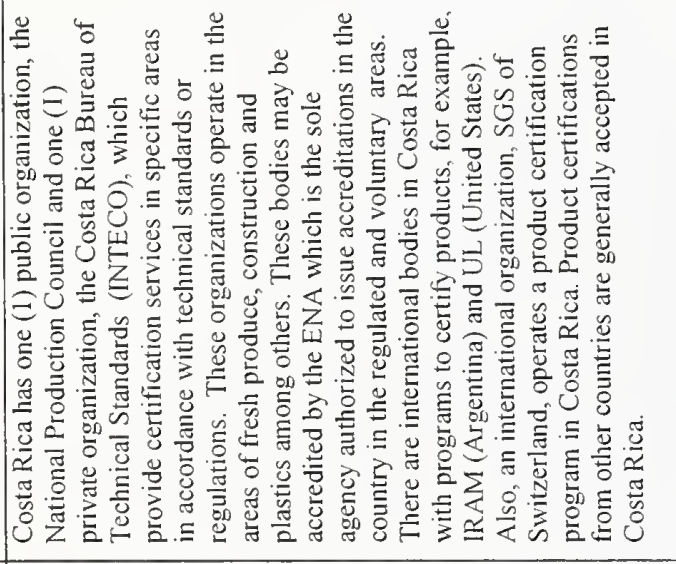 & 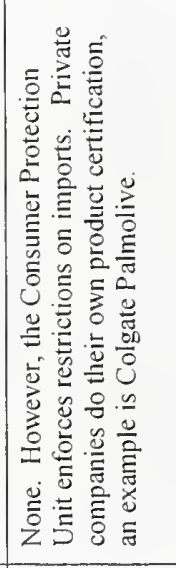 & 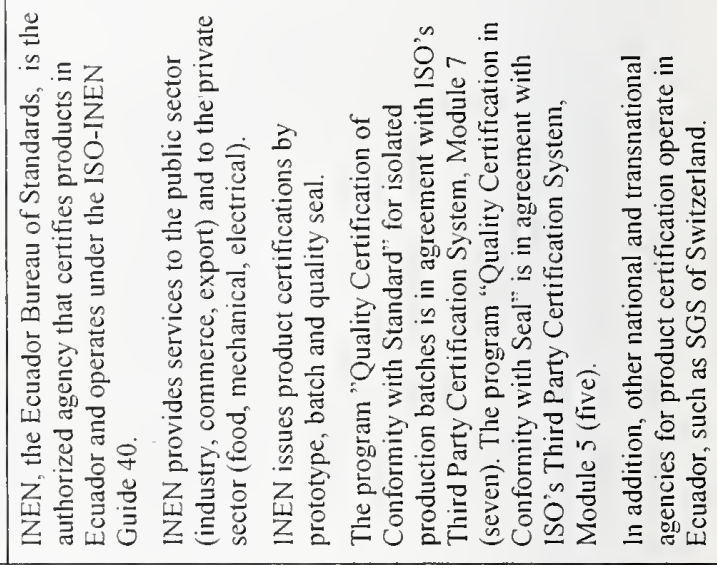 \\
\hline 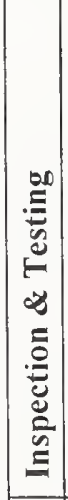 & 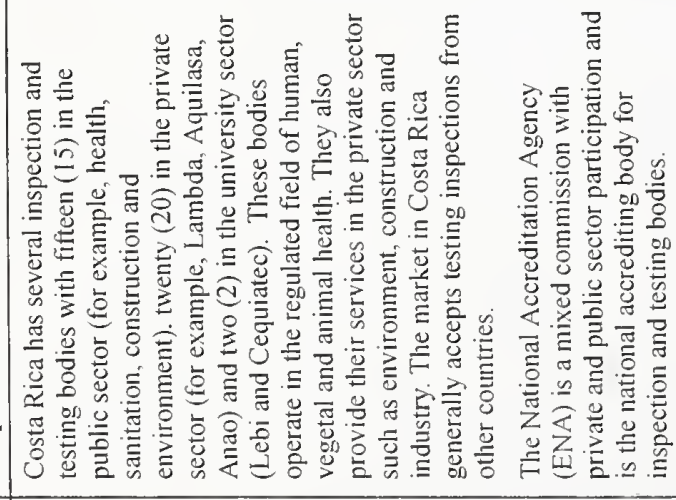 & 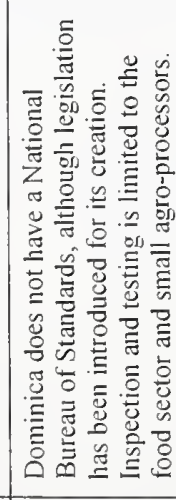 & 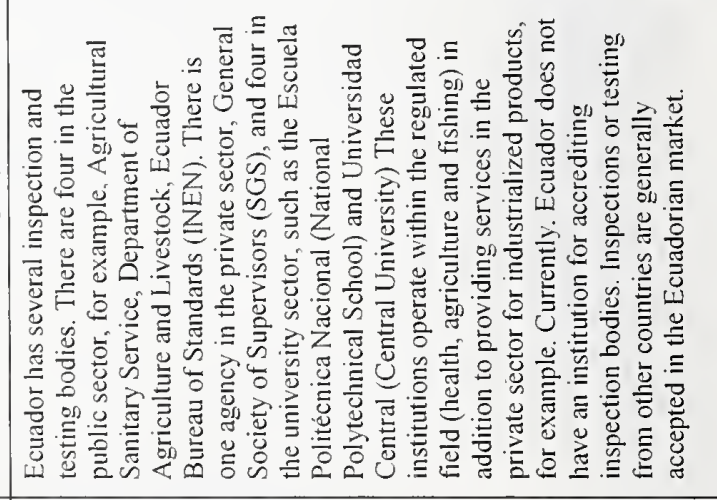 \\
\hline 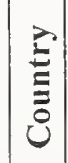 & 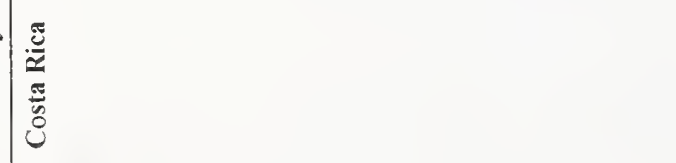 & 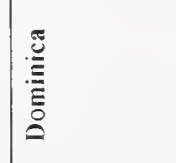 & 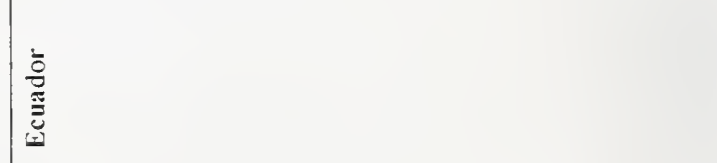 \\
\hline
\end{tabular}




\begin{tabular}{|c|c|c|c|}
\hline 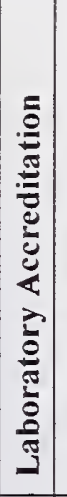 & 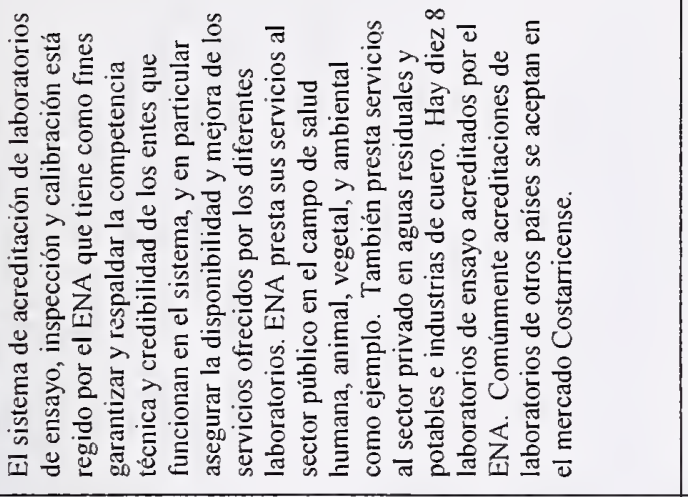 & 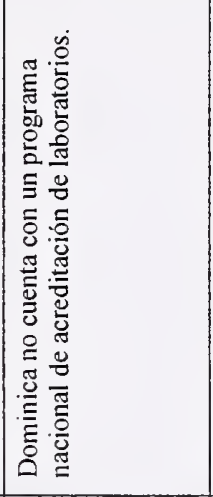 & 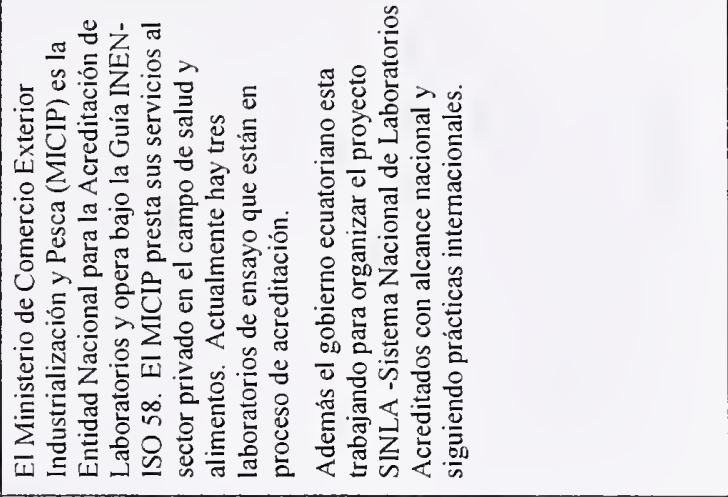 \\
\hline 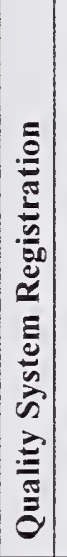 & 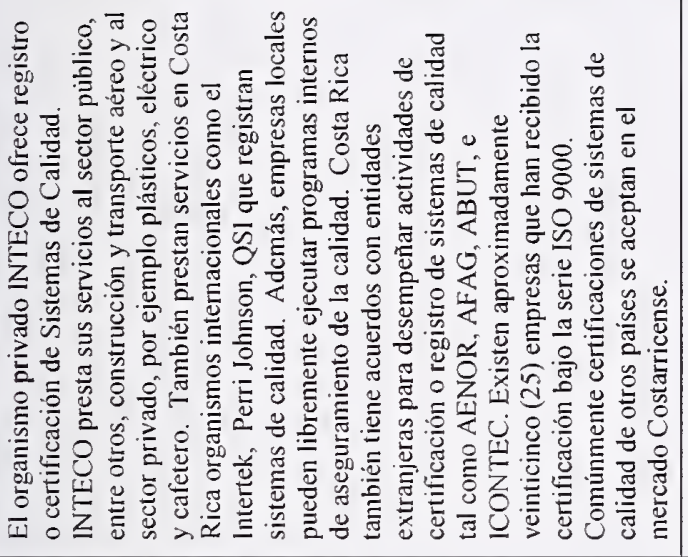 & 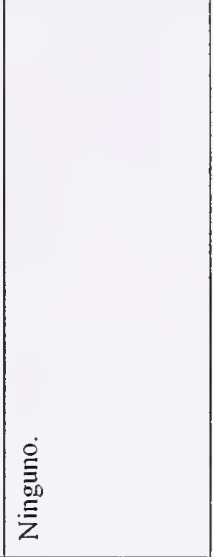 & 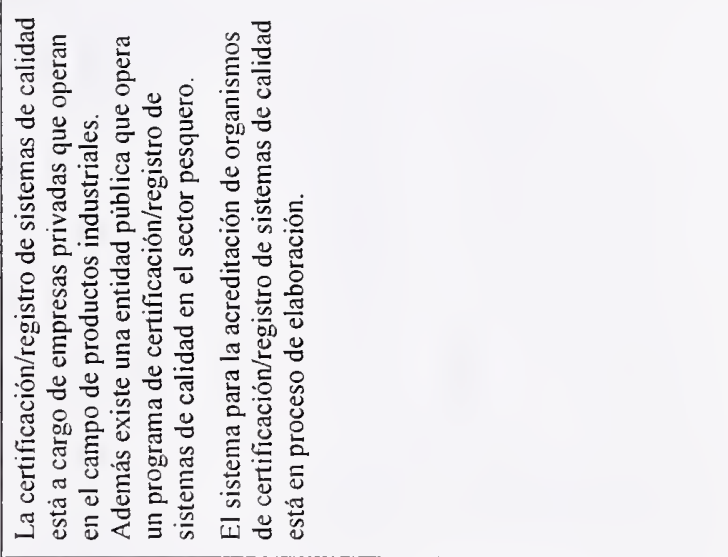 \\
\hline 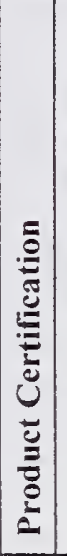 & 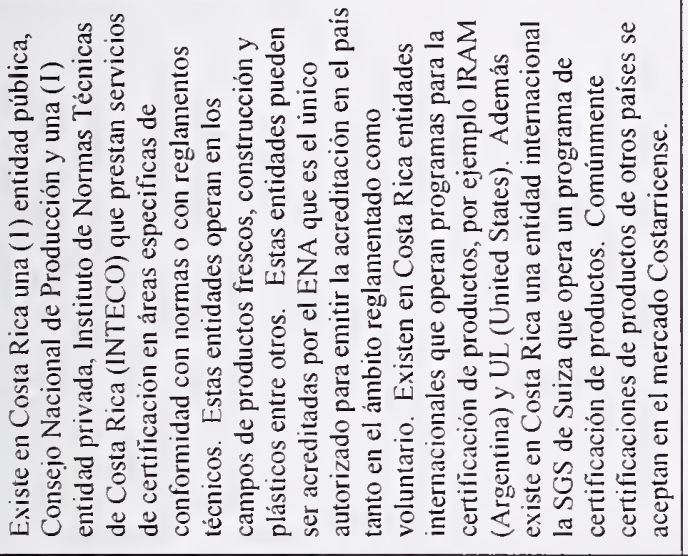 & 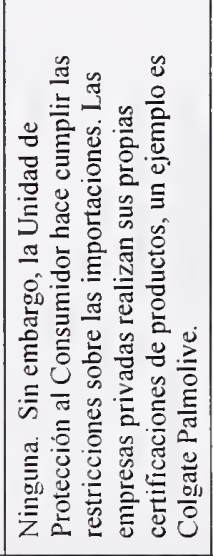 & 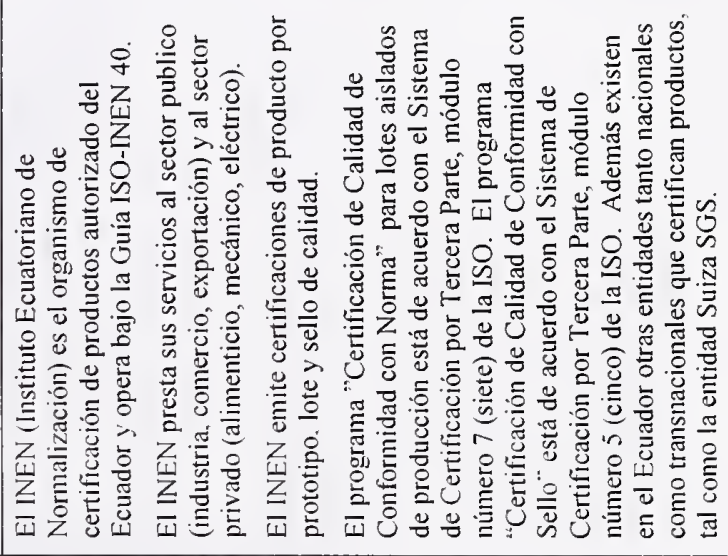 \\
\hline 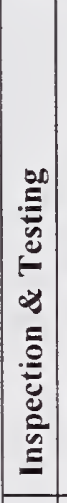 & 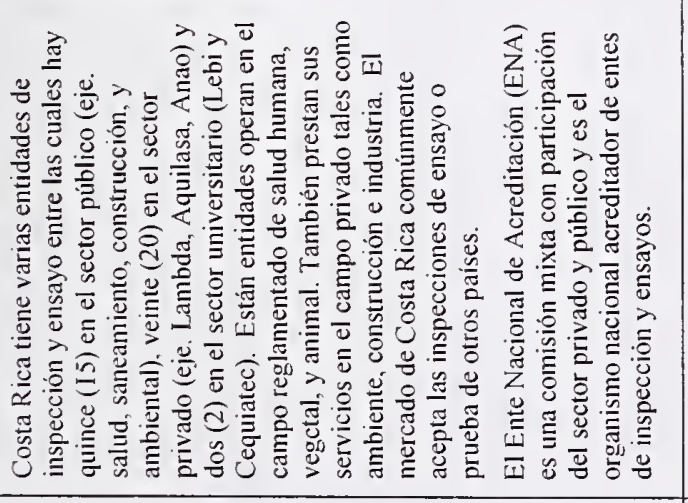 & 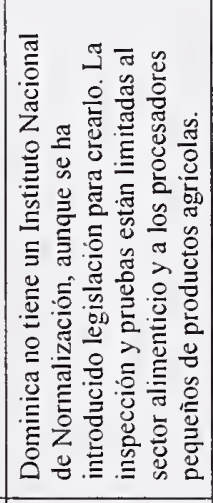 & 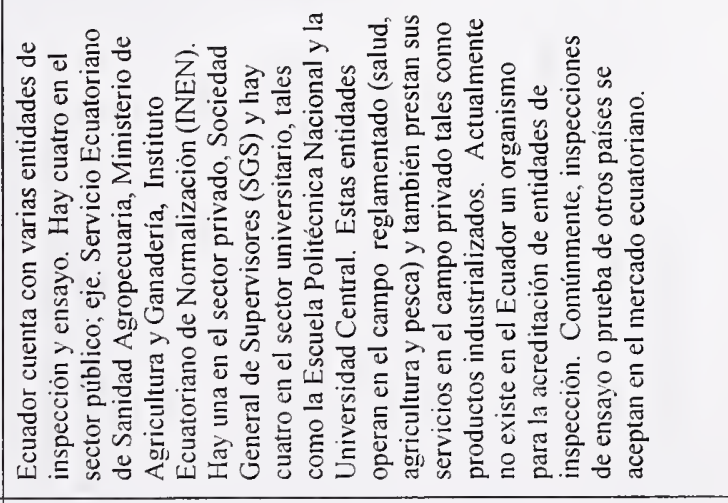 \\
\hline $\begin{array}{l}\vec{\Xi} \\
\stackrel{\Xi}{\Xi} \\
\dot{\Xi}\end{array}$ & 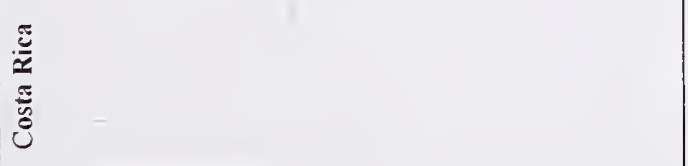 & Ĕ & \\
\hline
\end{tabular}




\begin{tabular}{|c|c|c|}
\hline 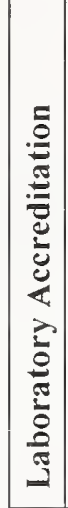 & 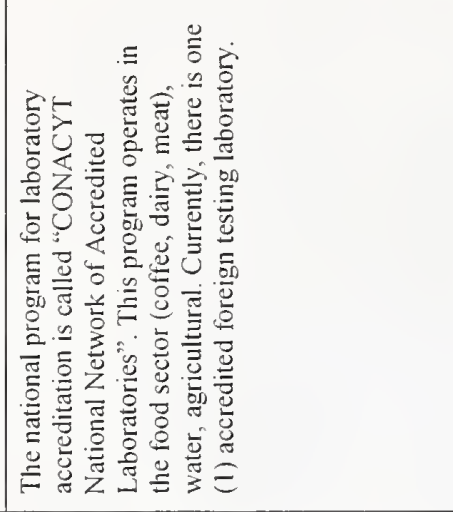 & 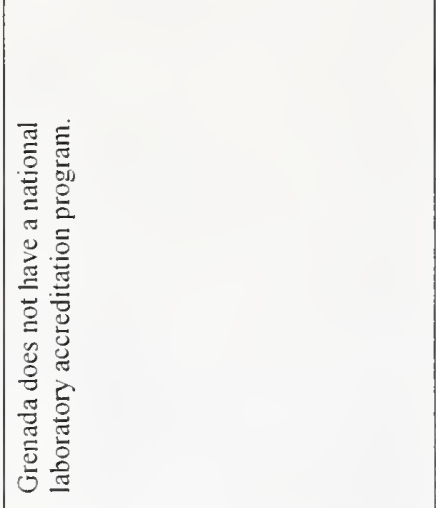 \\
\hline 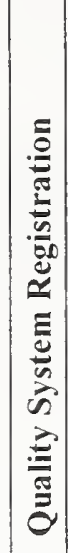 & 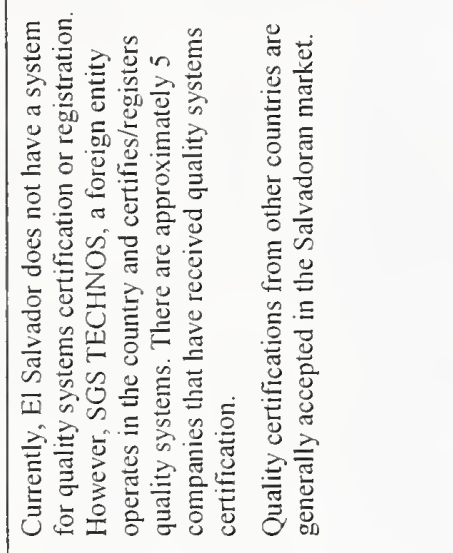 & 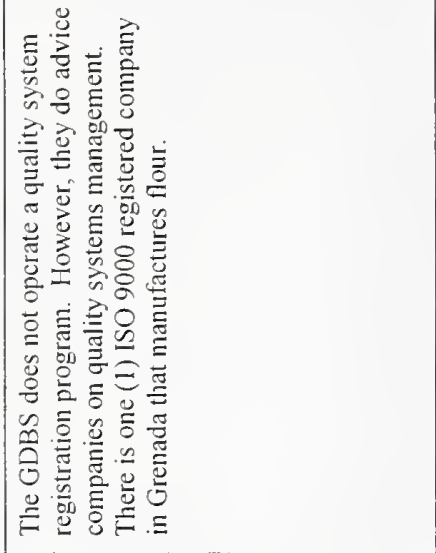 \\
\hline 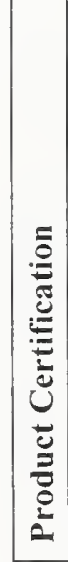 & 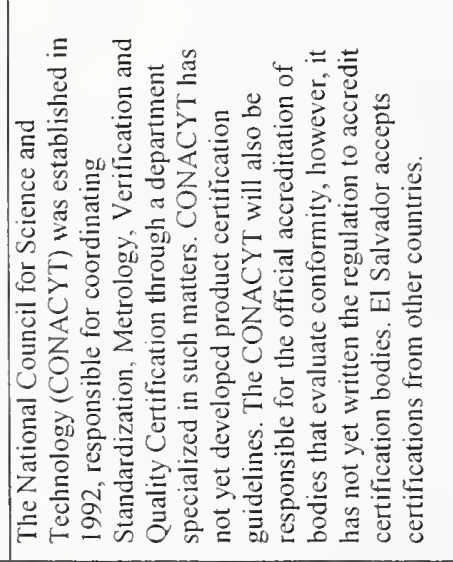 & 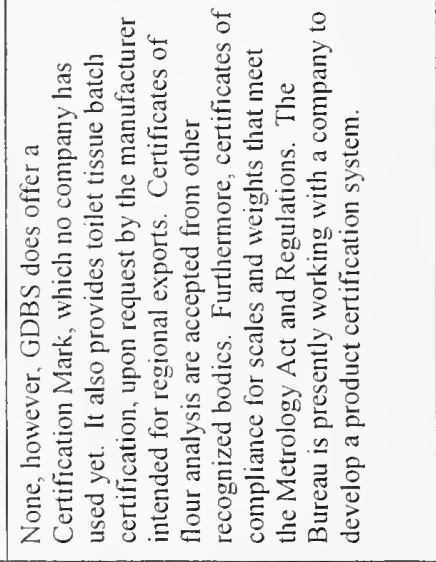 \\
\hline 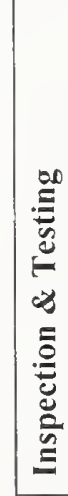 & 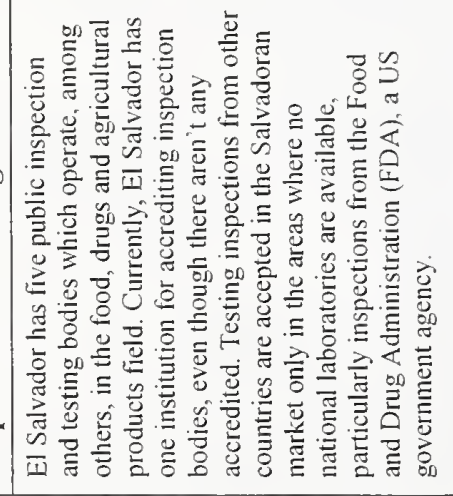 & 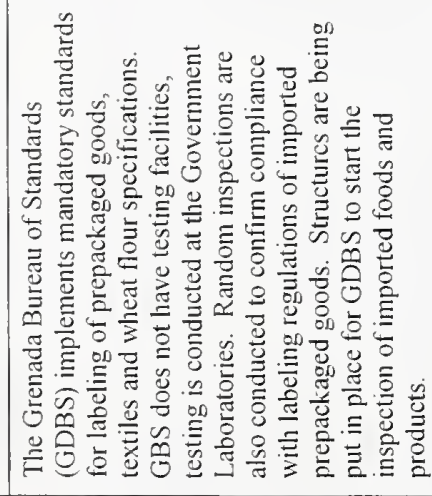 \\
\hline & & \\
\hline
\end{tabular}




\begin{tabular}{|c|c|c|}
\hline 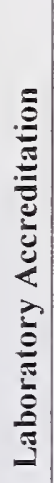 & 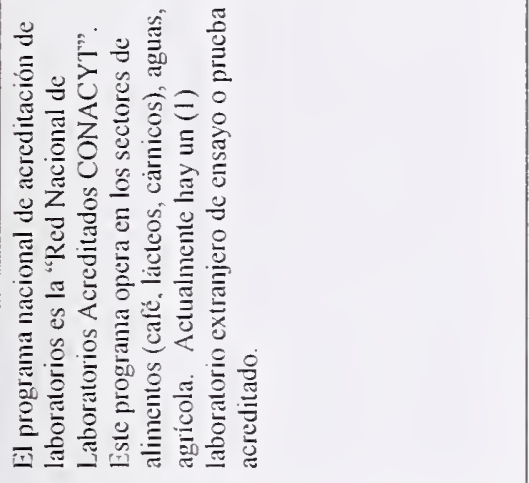 & 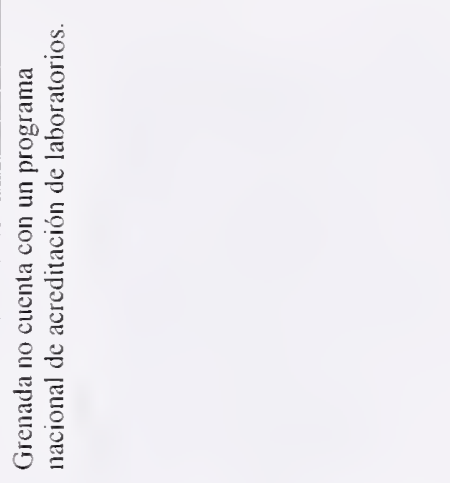 \\
\hline 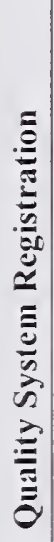 & 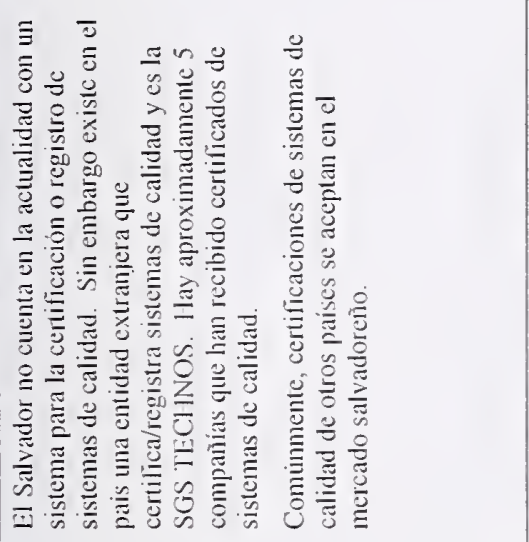 & 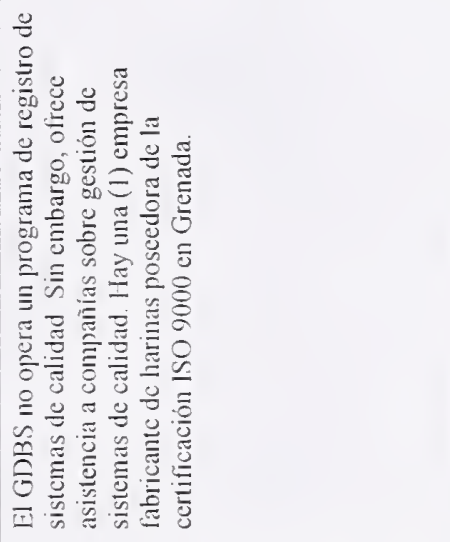 \\
\hline 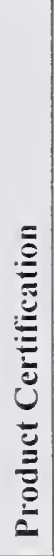 & 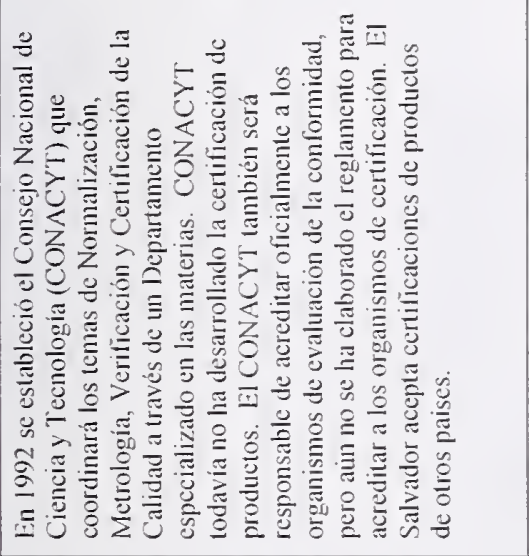 & 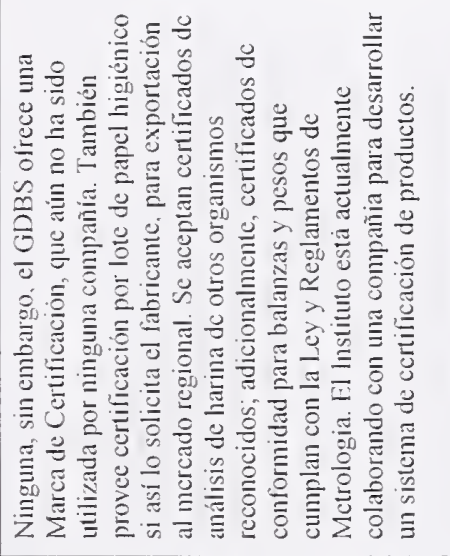 \\
\hline 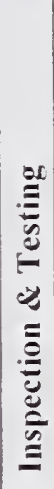 & 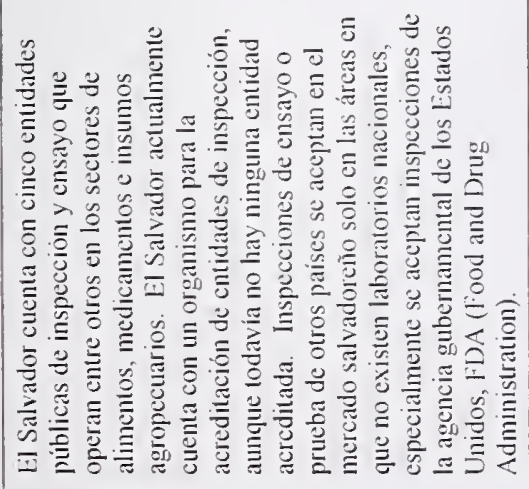 & 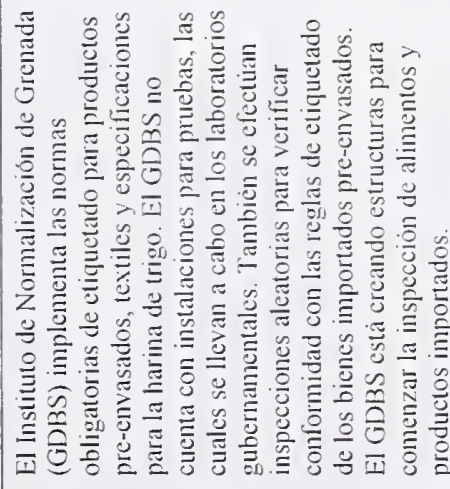 \\
\hline U્ & 吾 & \\
\hline
\end{tabular}




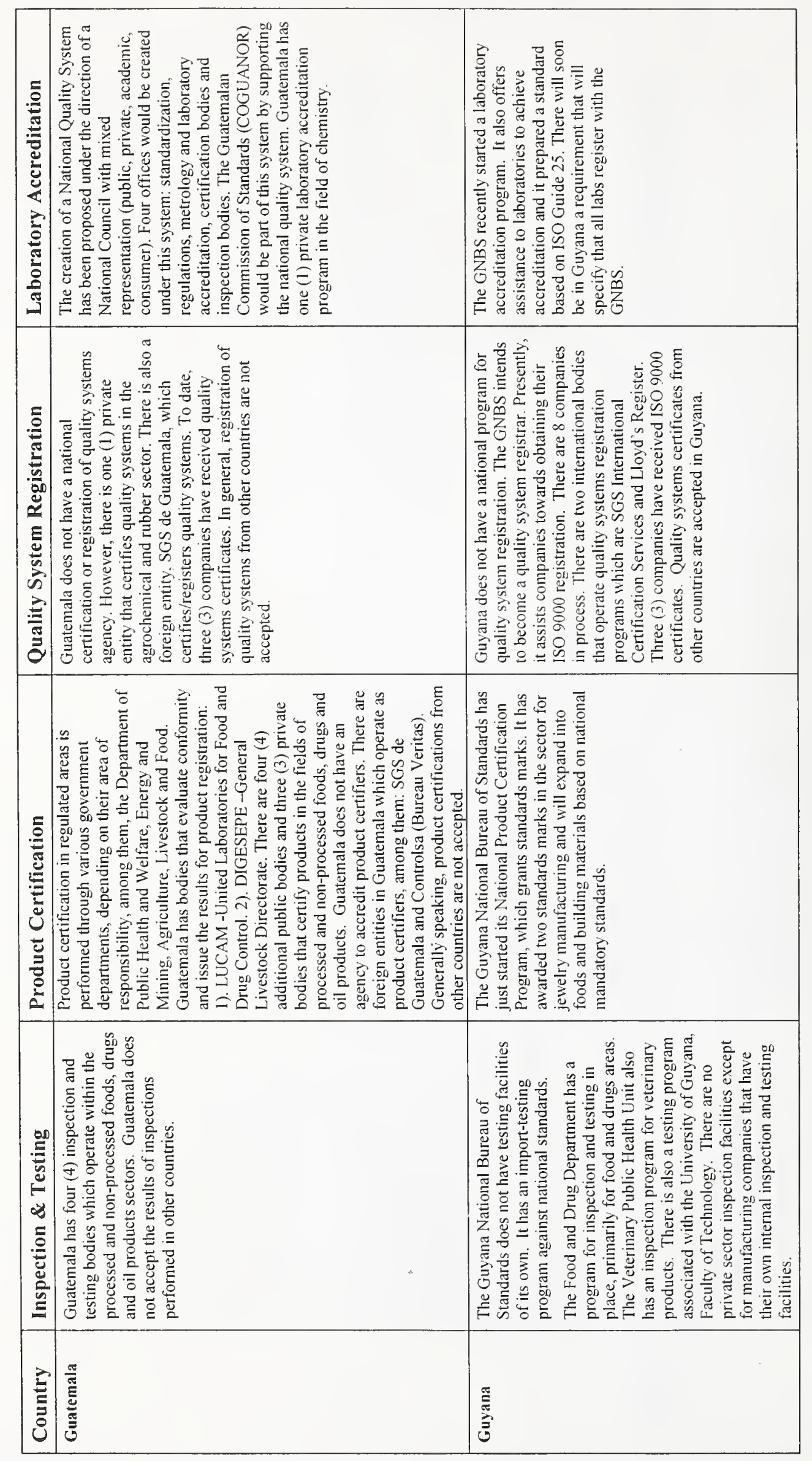




\begin{tabular}{|c|c|c|}
\hline 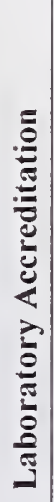 & 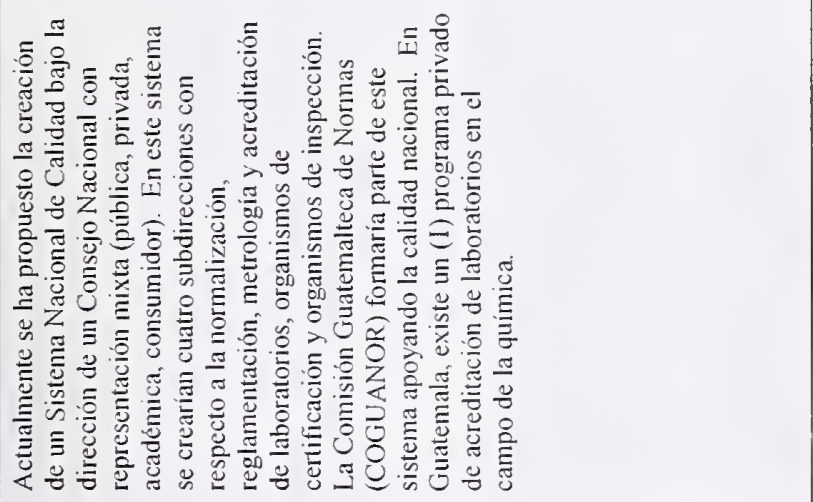 & 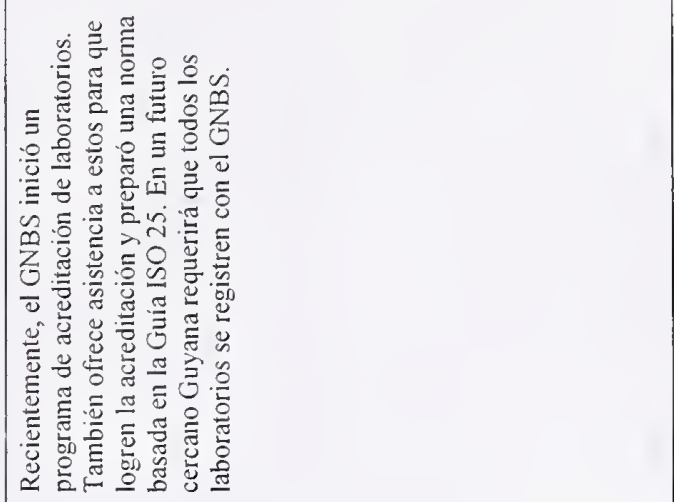 \\
\hline 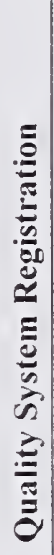 & 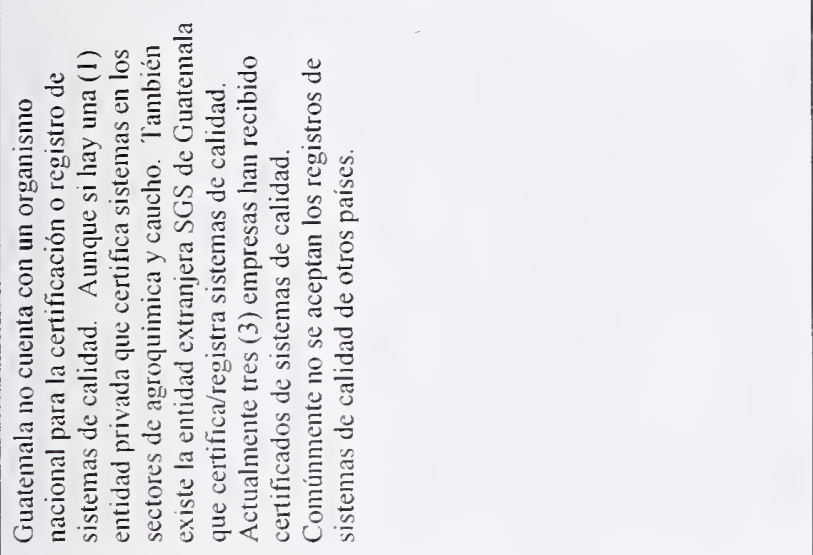 & 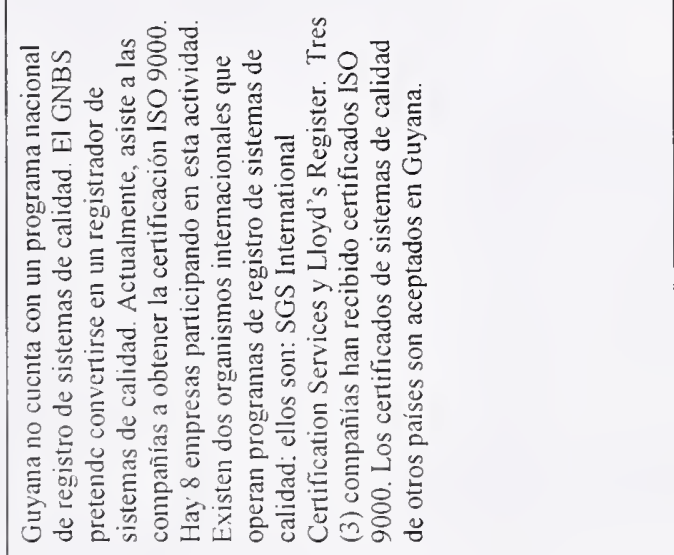 \\
\hline 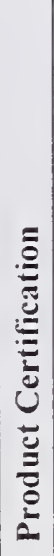 & 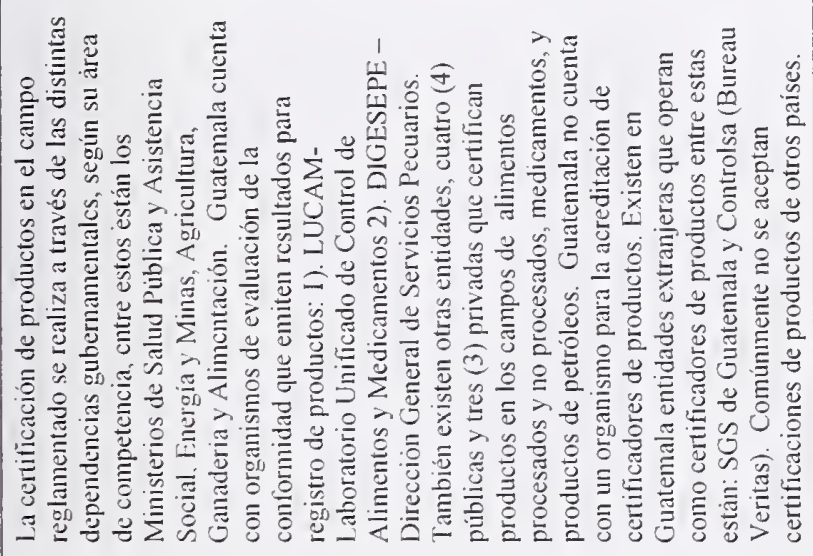 & 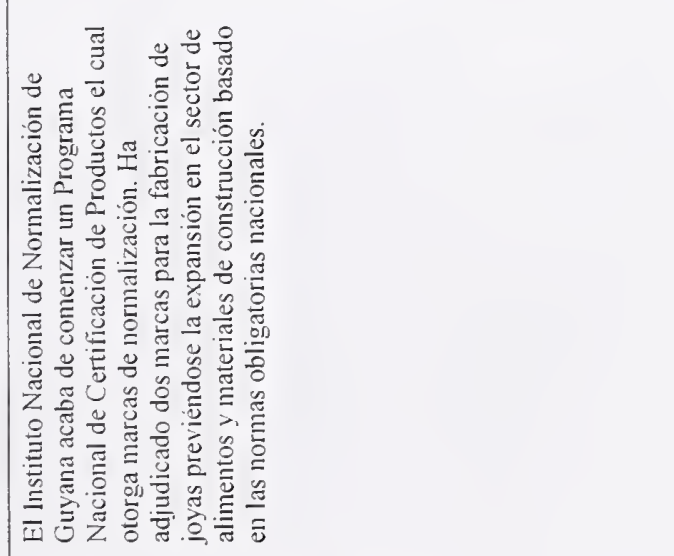 \\
\hline 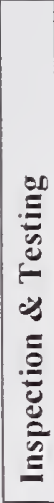 & 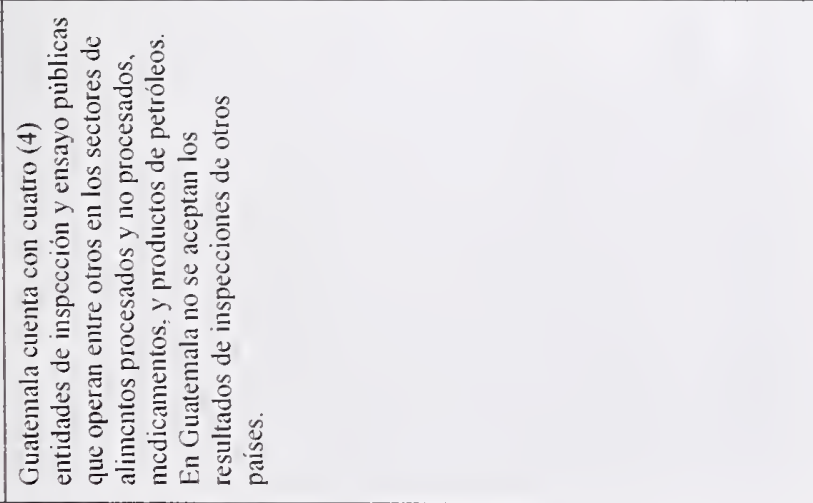 & 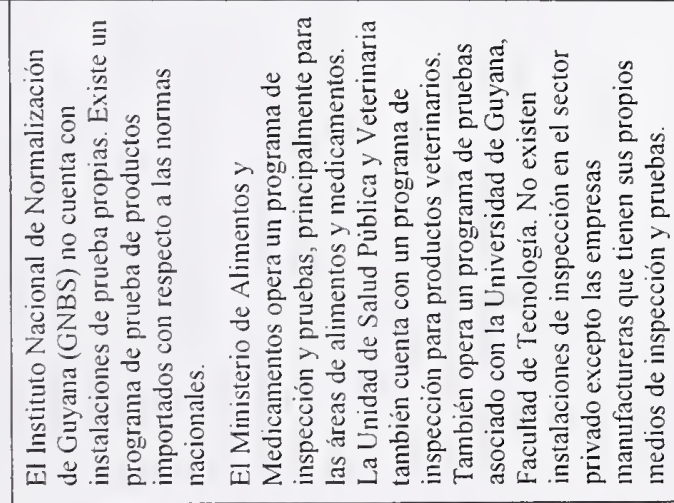 \\
\hline$\stackrel{\Xi}{\Xi}$ & & $\stackrel{\bar{N}}{\mathrm{~N}}$ \\
\hline
\end{tabular}




\begin{tabular}{|c|c|c|}
\hline 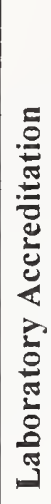 & 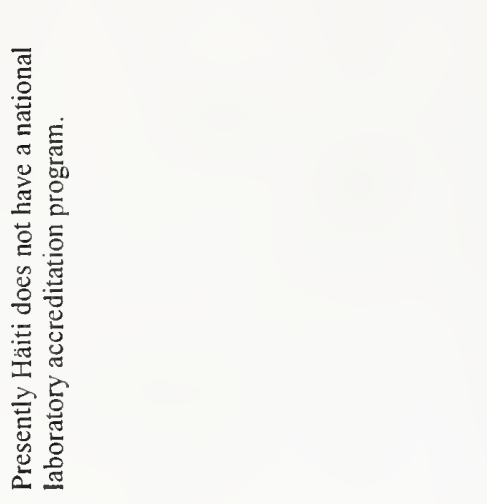 & 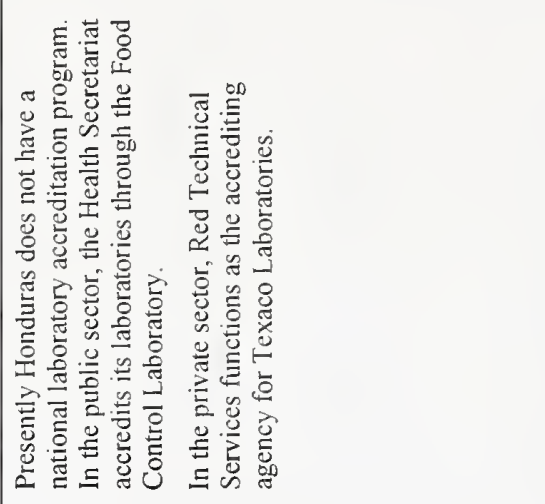 \\
\hline 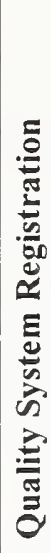 & 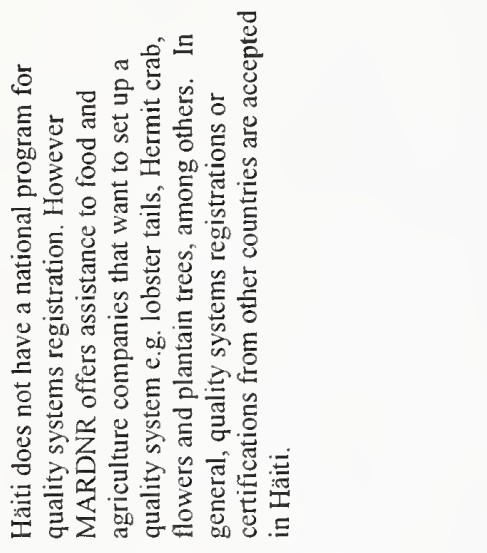 & 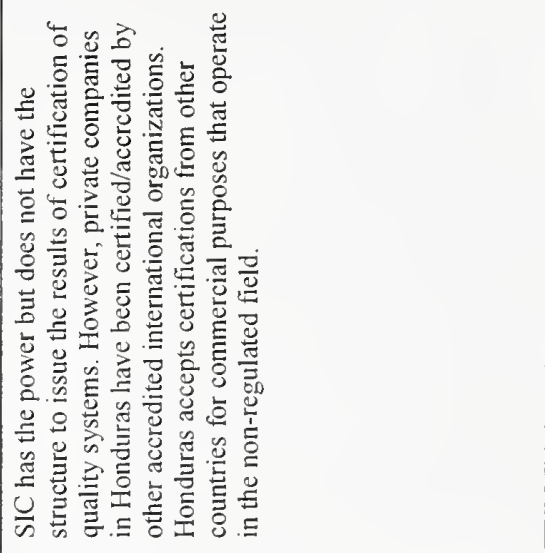 \\
\hline & 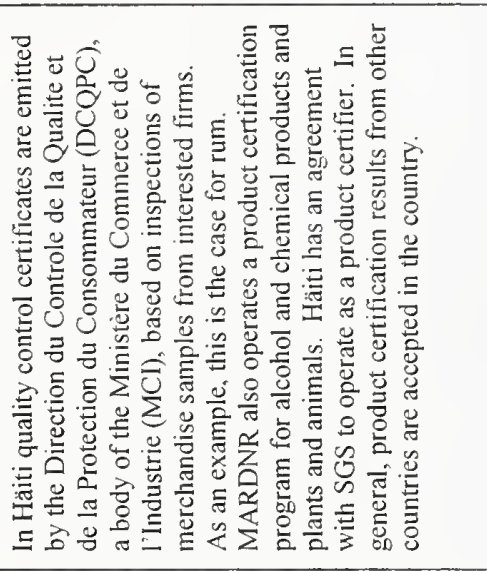 & 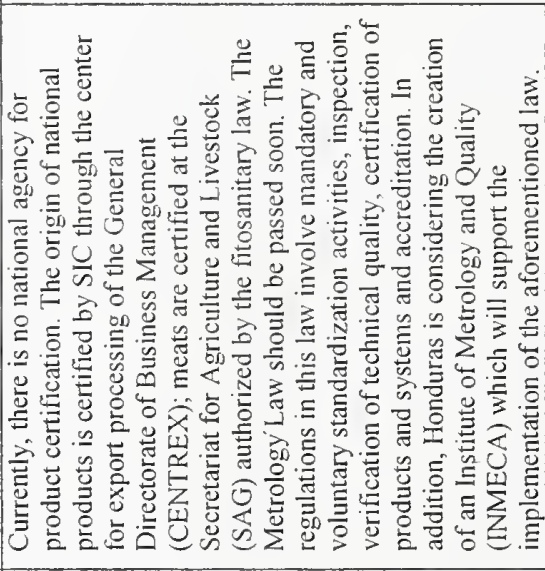 \\
\hline & 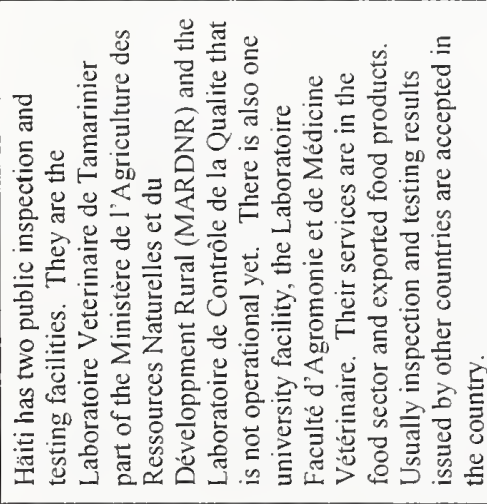 & 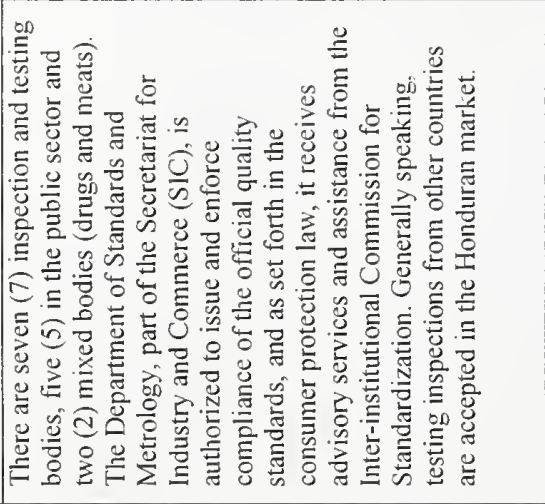 \\
\hline 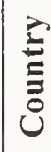 & & \\
\hline
\end{tabular}




\begin{tabular}{|c|c|c|}
\hline 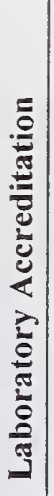 & 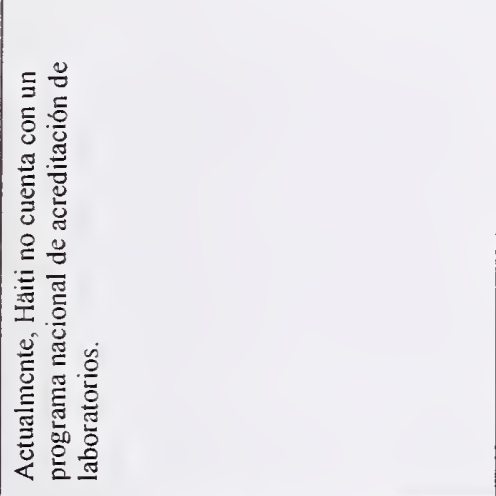 & 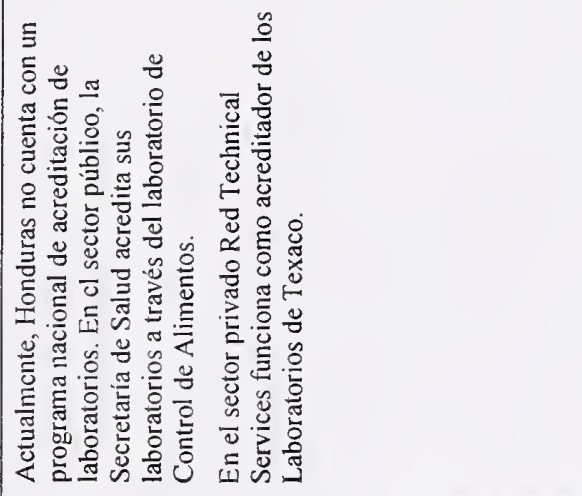 \\
\hline 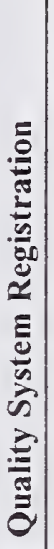 & 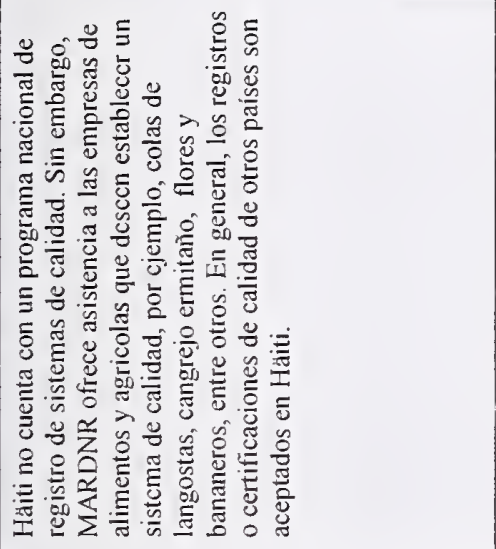 & 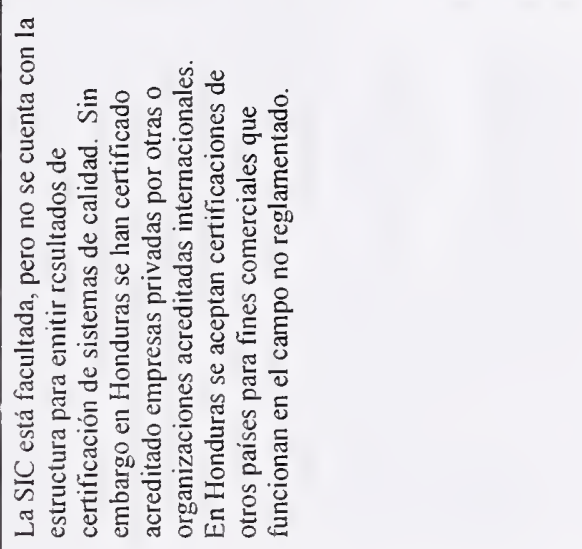 \\
\hline & 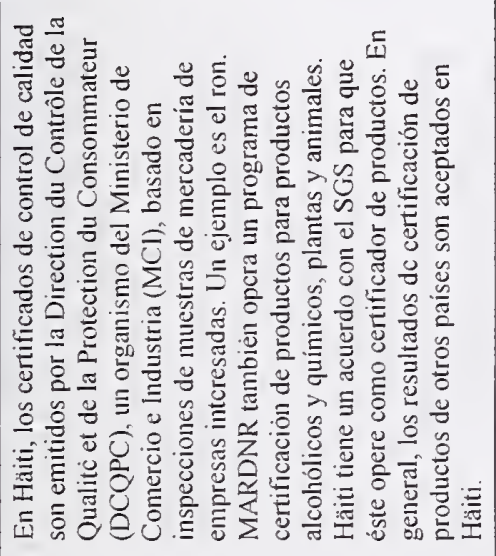 & 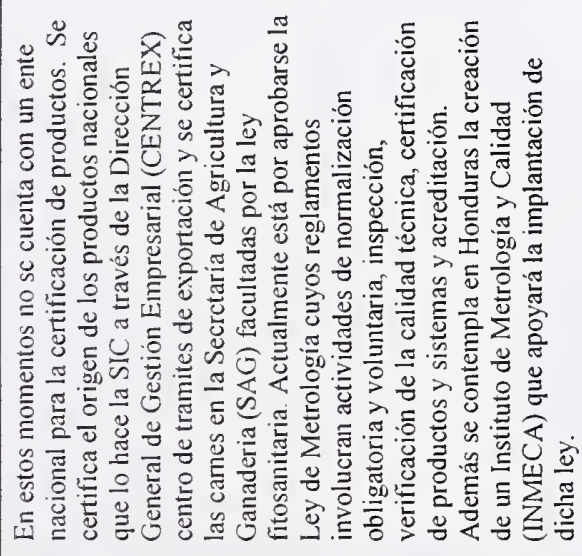 \\
\hline 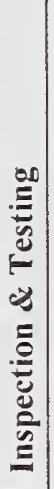 & 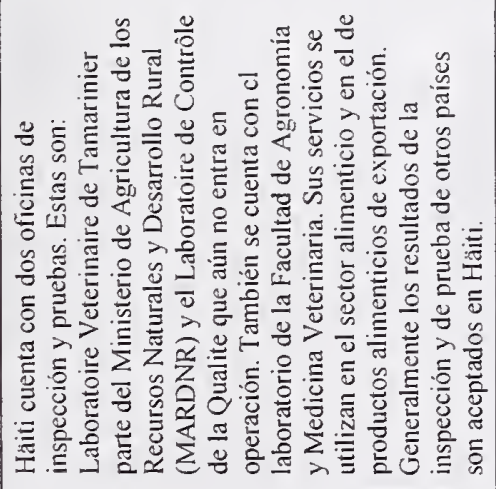 & 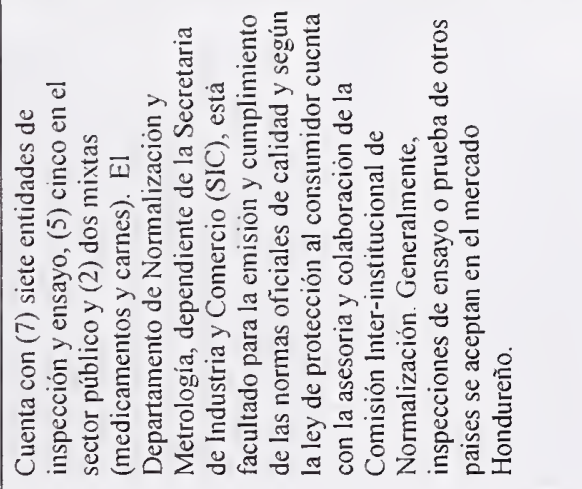 \\
\hline & $\overline{\bar{E}}$ & \\
\hline
\end{tabular}




\begin{tabular}{|c|c|c|}
\hline & 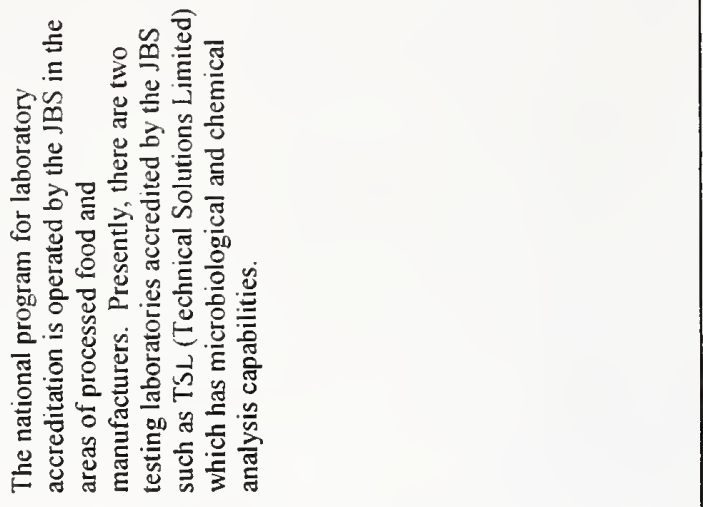 & 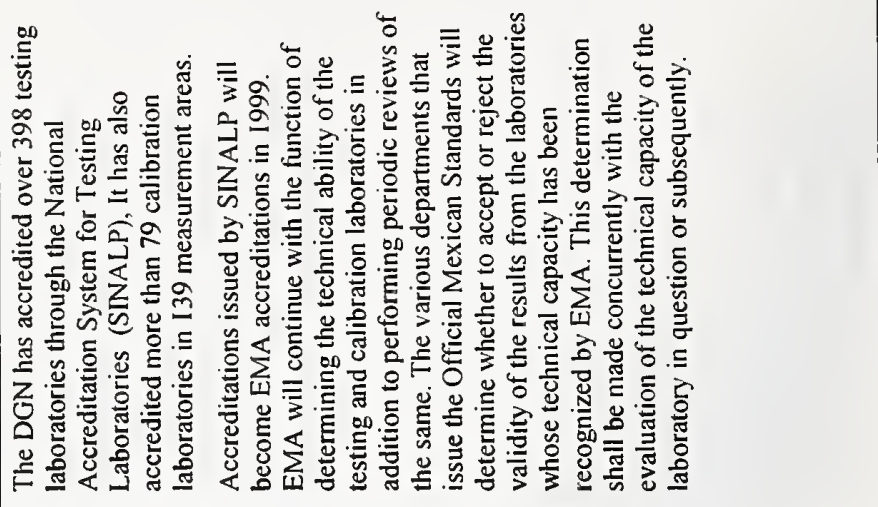 \\
\hline & 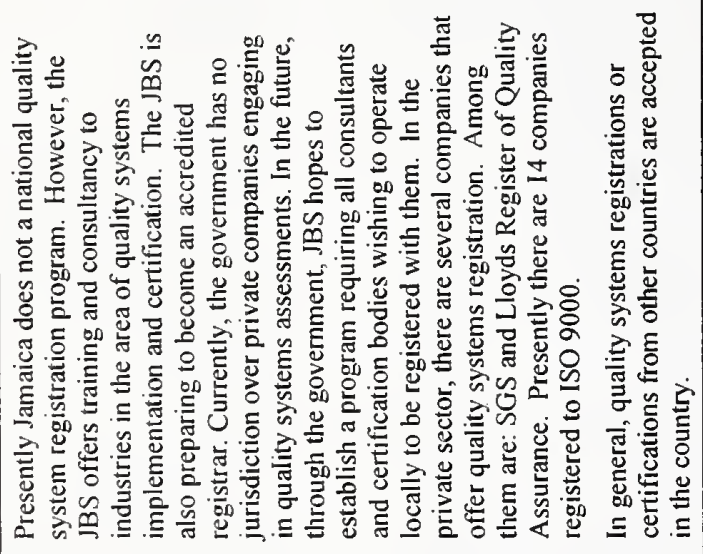 & 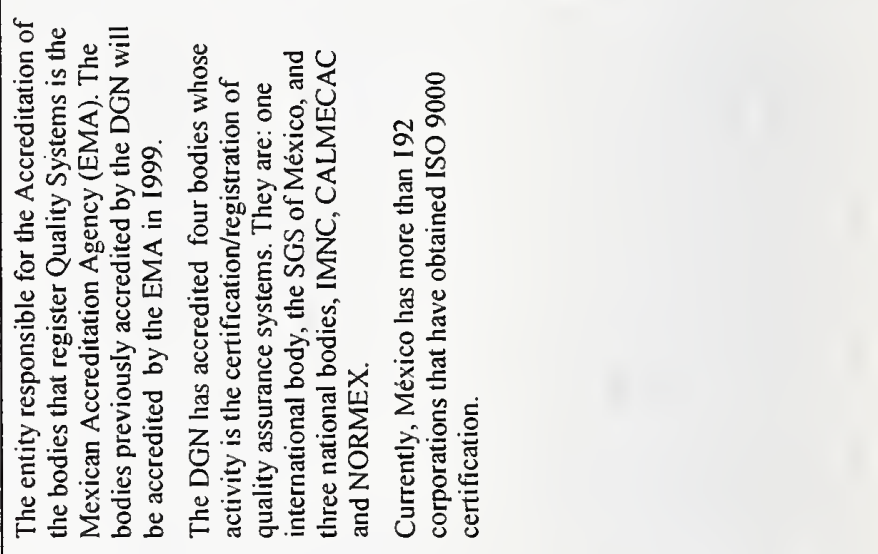 \\
\hline $\begin{array}{l}0 \\
0 \\
0 \\
0\end{array}$ & 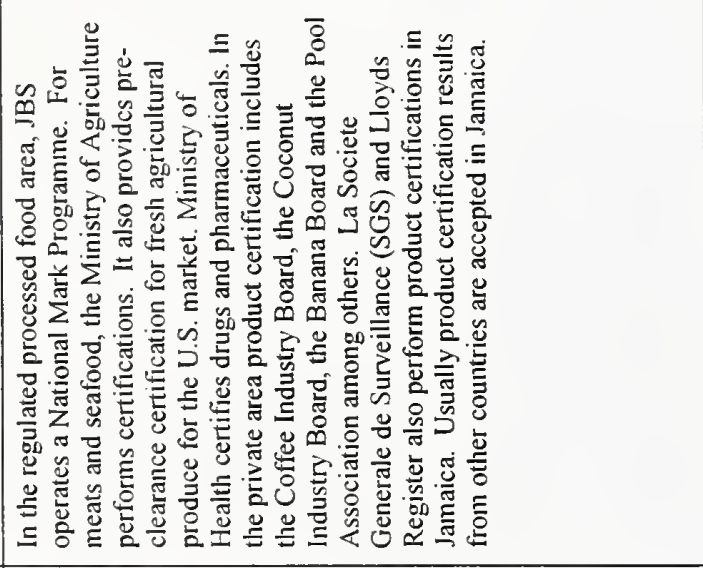 & 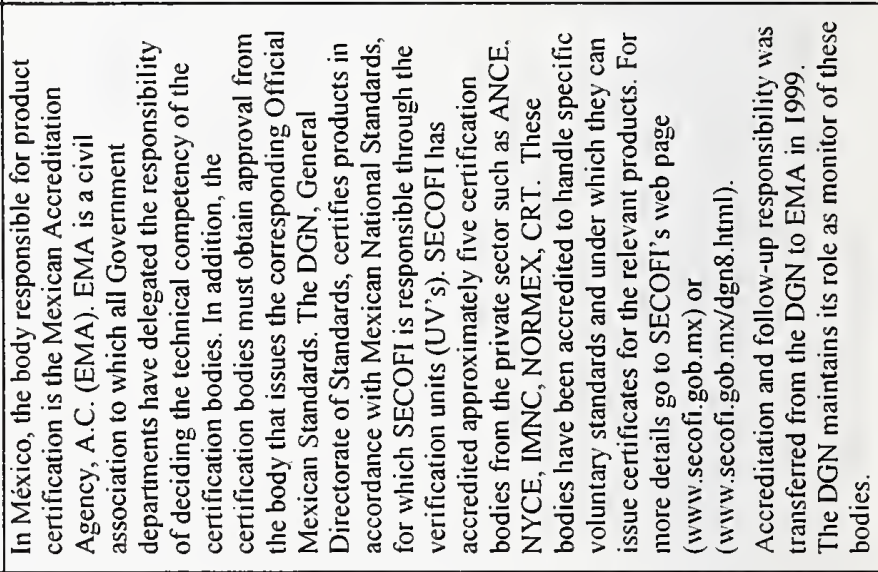 \\
\hline 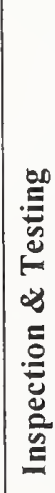 & 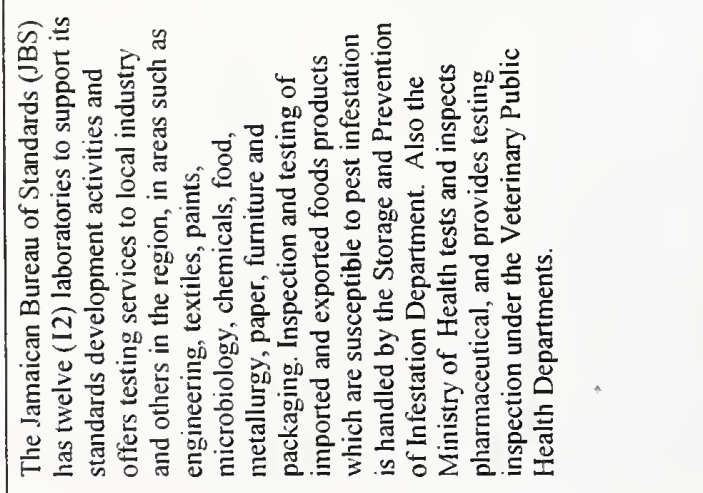 & 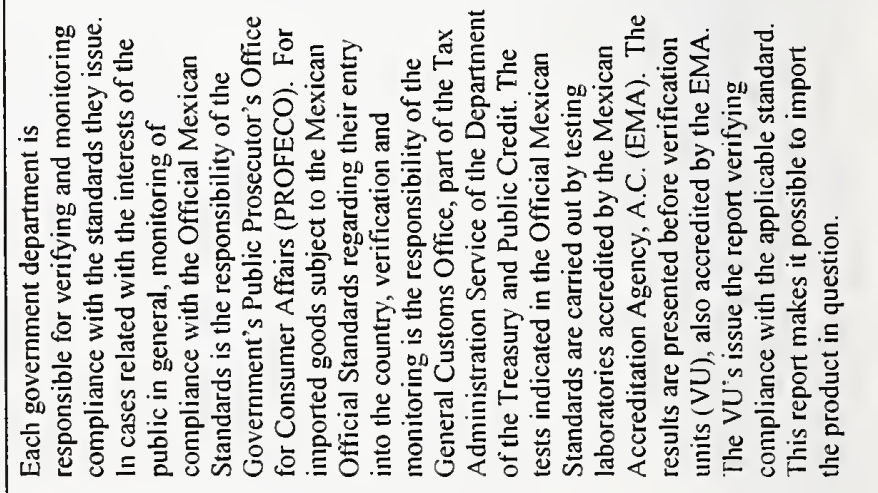 \\
\hline & & \\
\hline
\end{tabular}




\begin{tabular}{|c|c|c|}
\hline 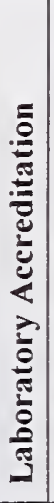 & 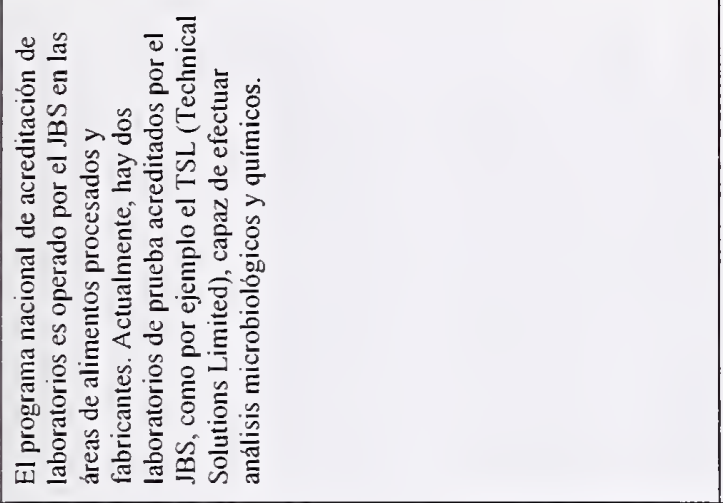 & 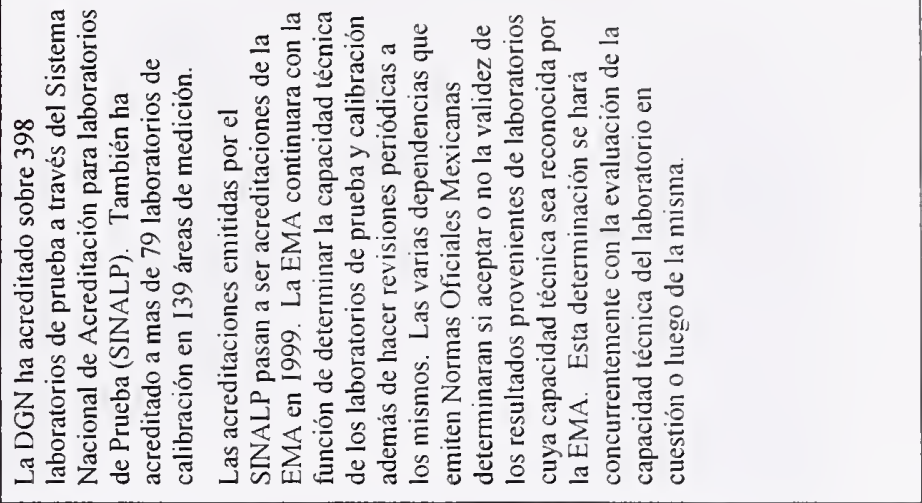 \\
\hline 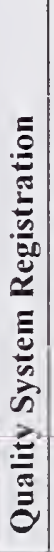 & 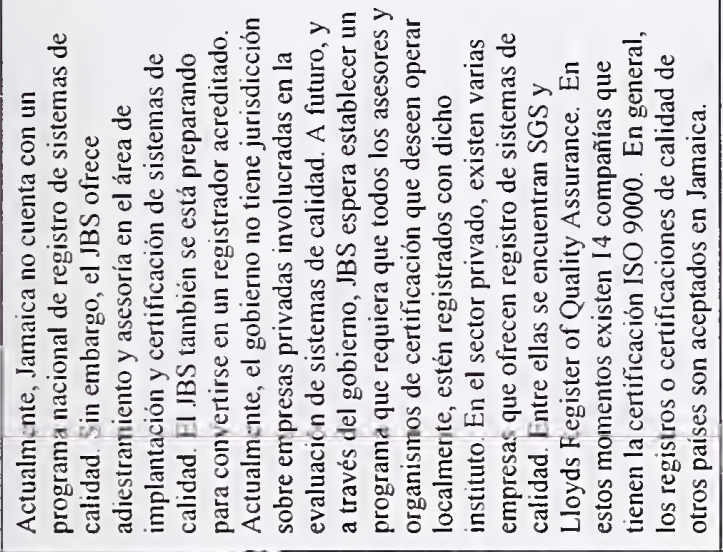 & 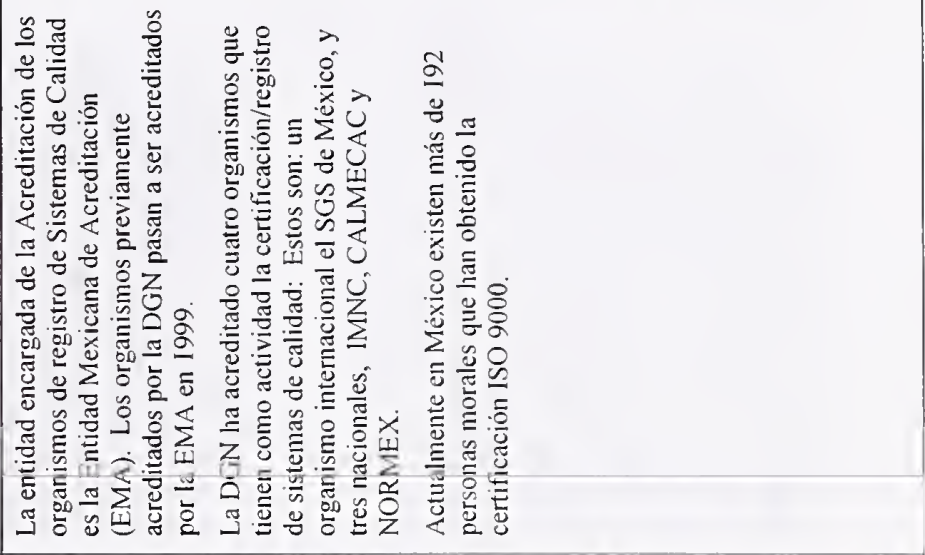 \\
\hline & 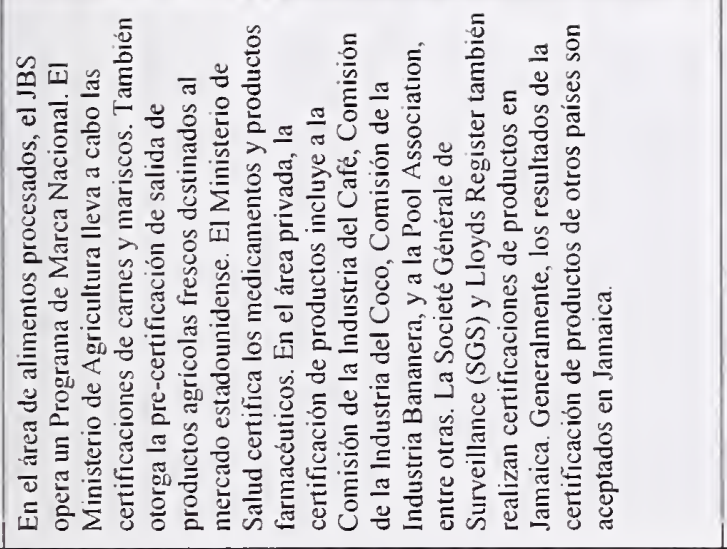 & 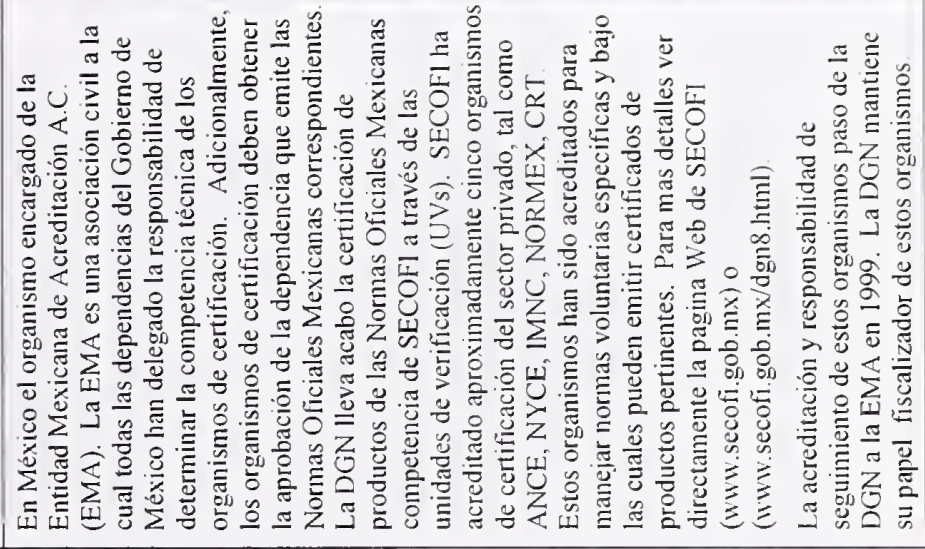 \\
\hline 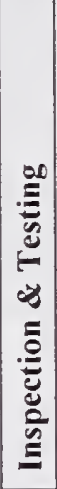 & 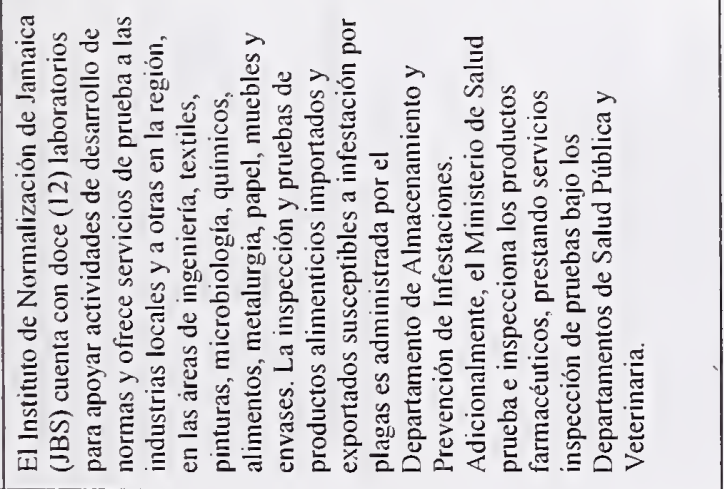 & 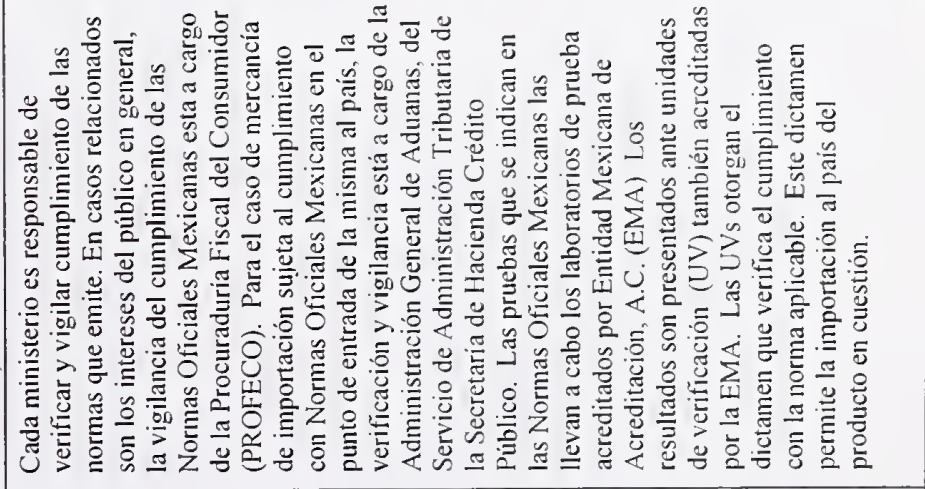 \\
\hline 良 & & \\
\hline
\end{tabular}




\begin{tabular}{|c|c|c|c|}
\hline 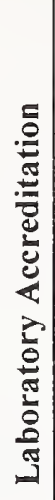 & 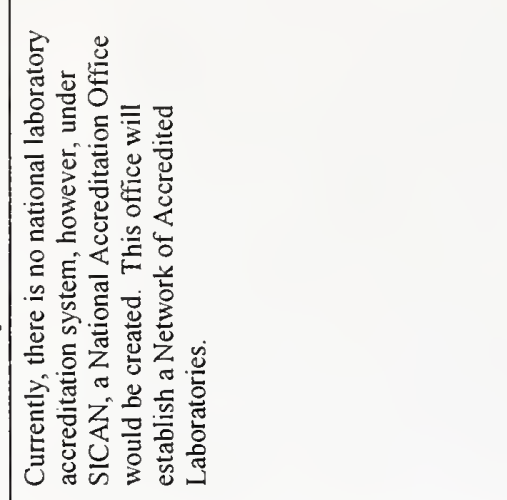 & 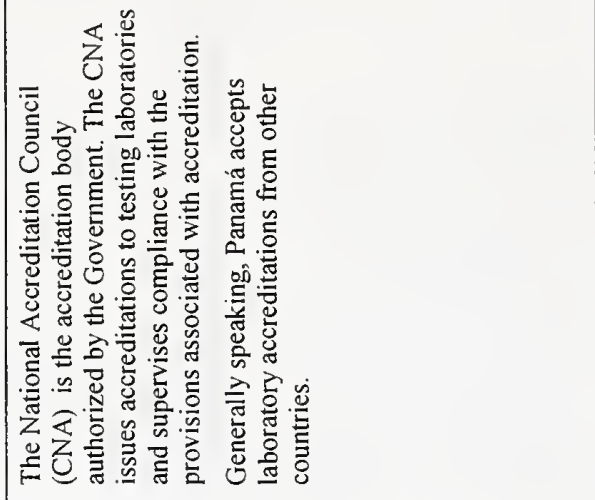 & 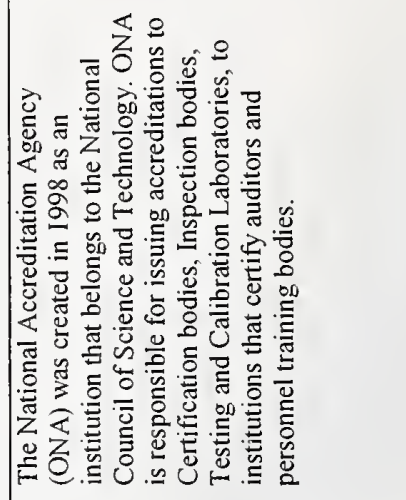 \\
\hline 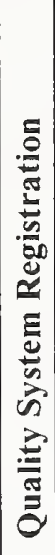 & 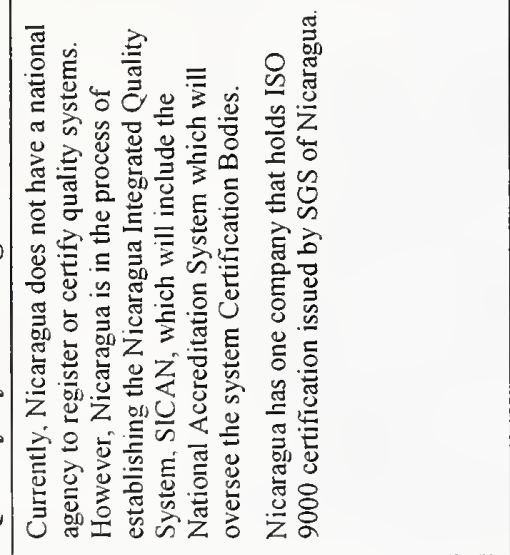 & 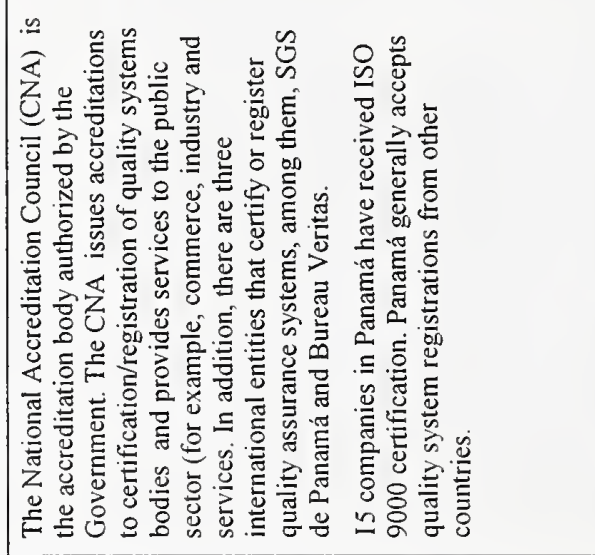 & 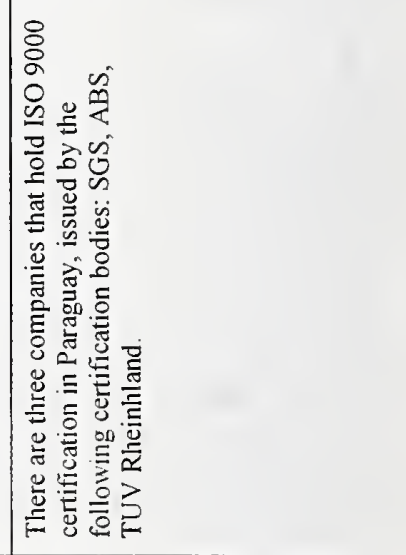 \\
\hline 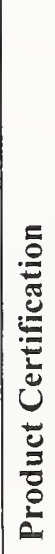 & 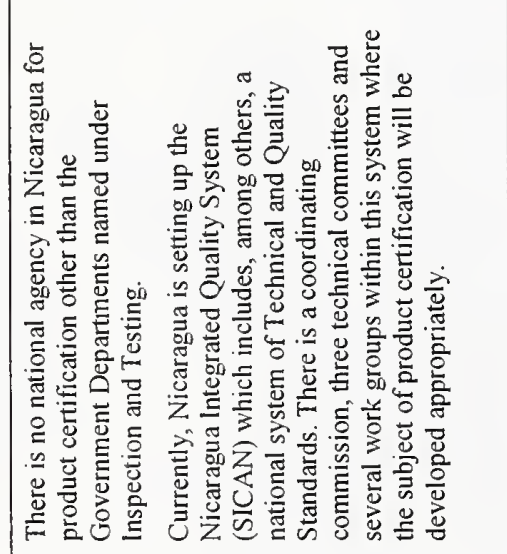 & 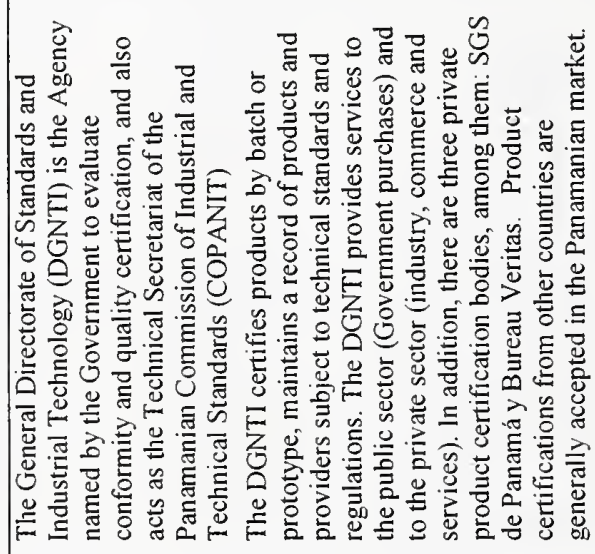 & 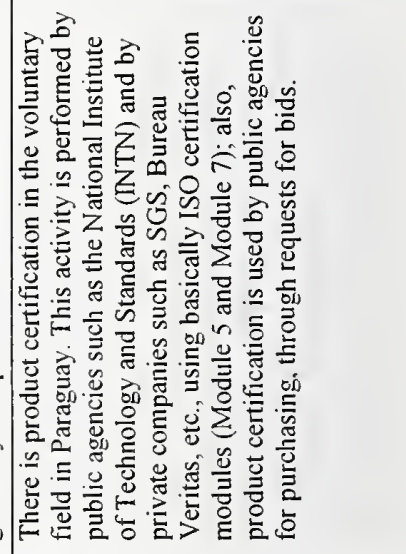 \\
\hline 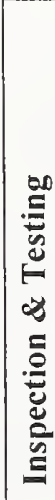 & 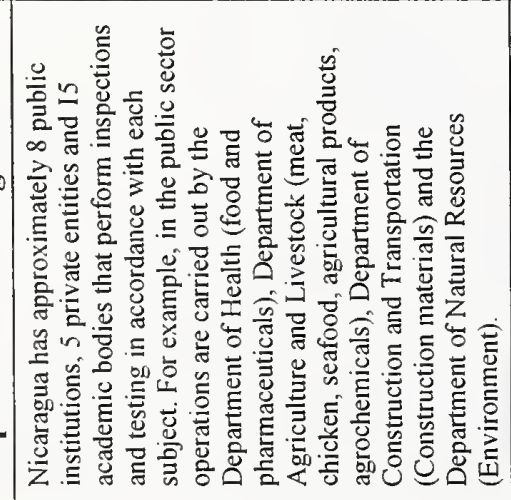 & 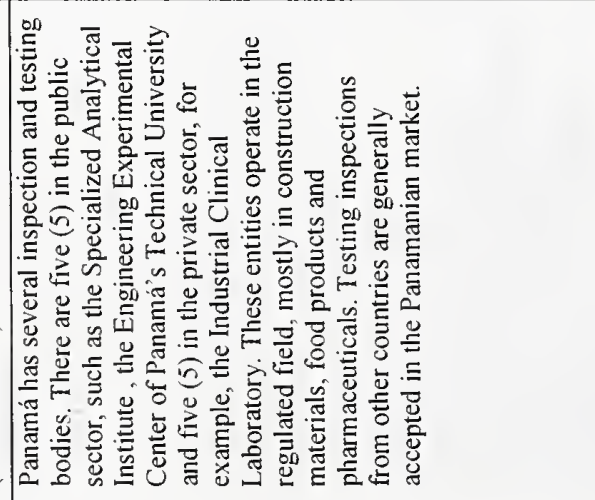 & 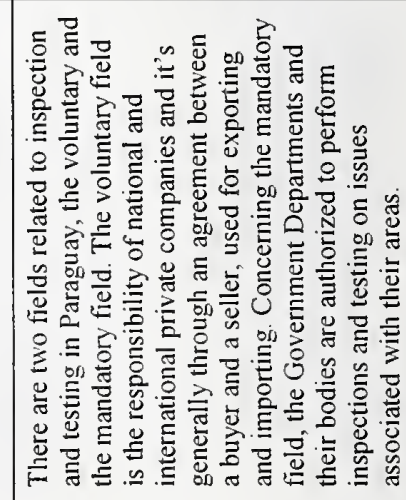 \\
\hline 递 & & 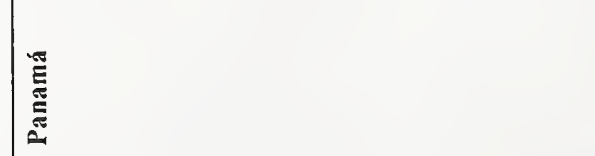 & 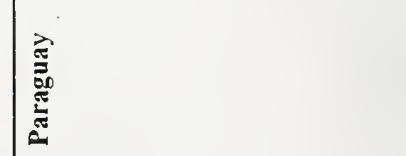 \\
\hline
\end{tabular}




\begin{tabular}{|c|c|c|c|}
\hline 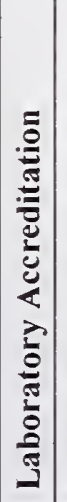 & 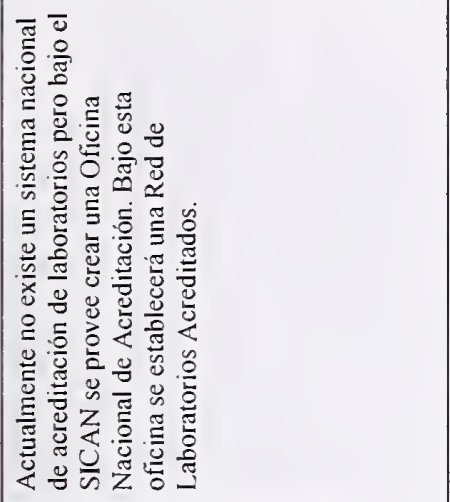 & 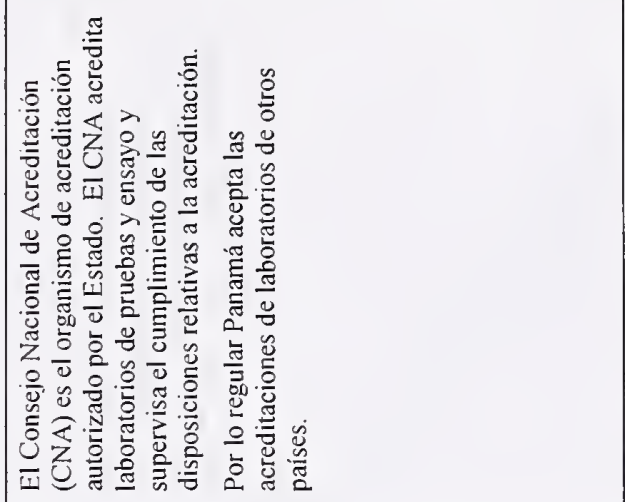 & 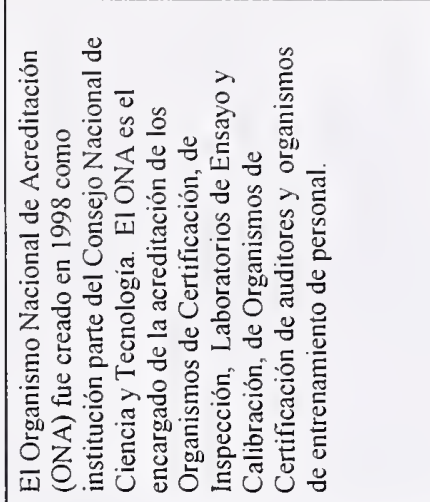 \\
\hline 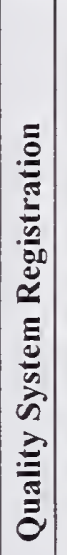 & 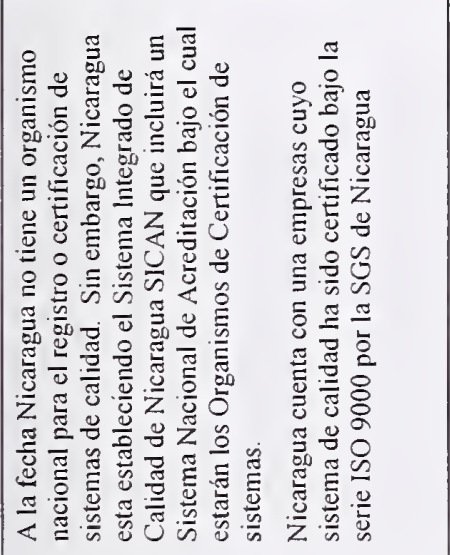 & 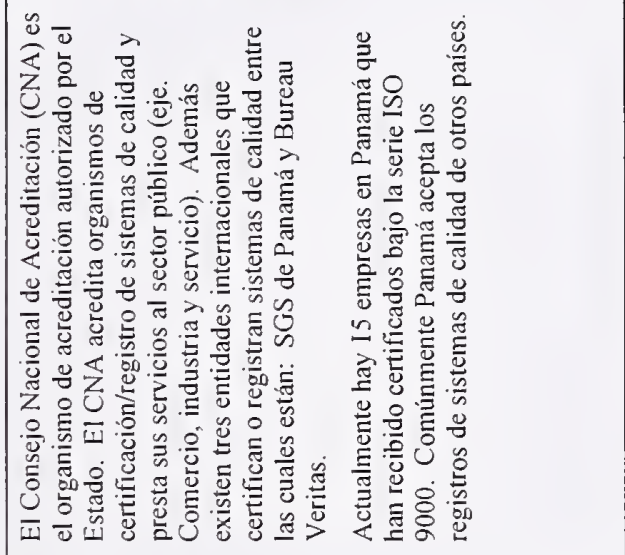 & 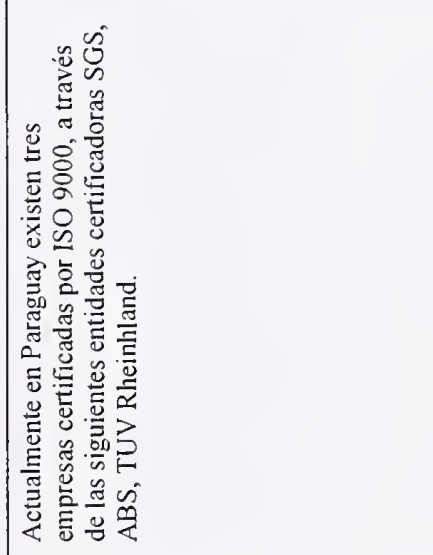 \\
\hline 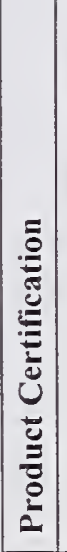 & 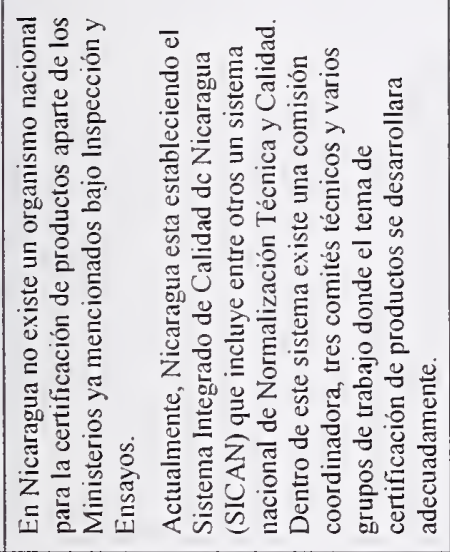 & 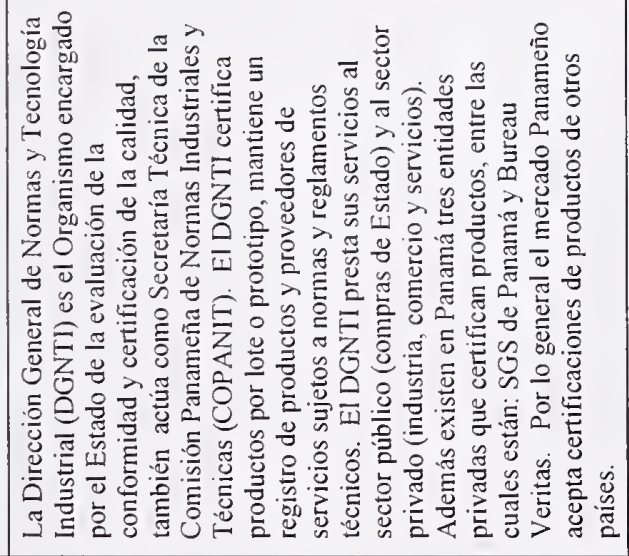 & 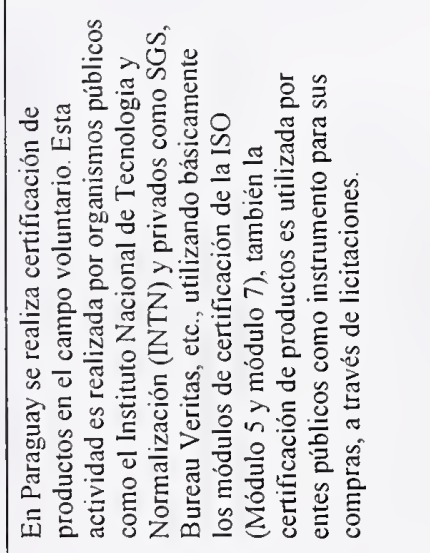 \\
\hline 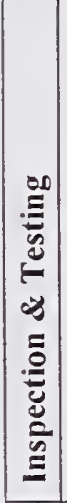 & 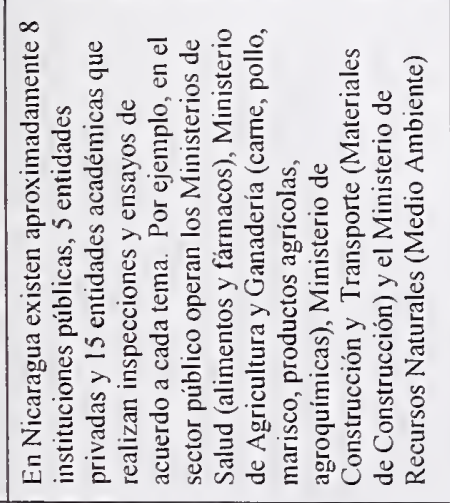 & 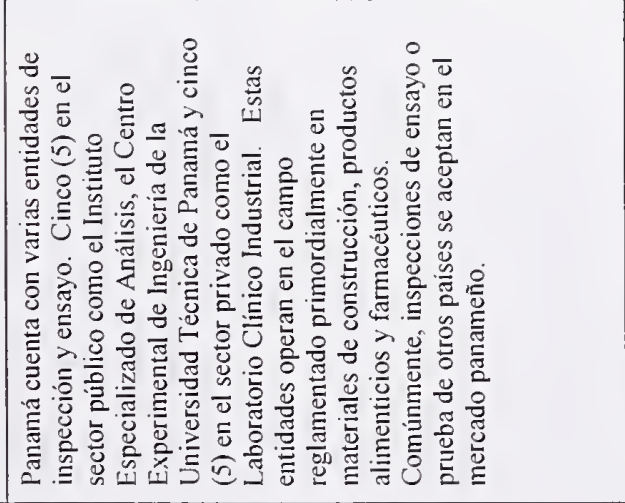 & 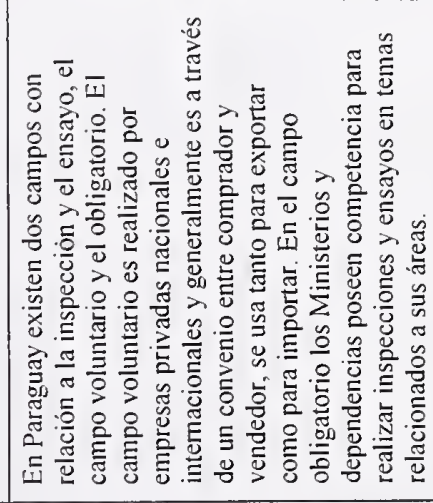 \\
\hline 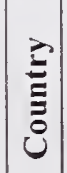 & 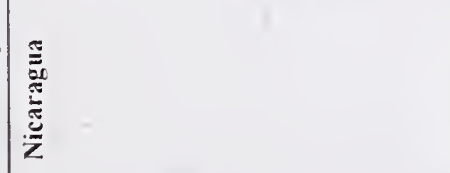 & $\begin{array}{l}\text { 焉 } \\
\text { 离 } \\
\text { 离 }\end{array}$ & 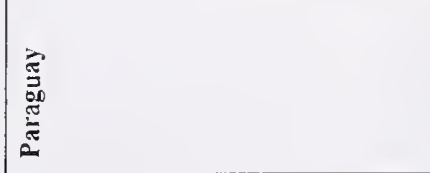 \\
\hline
\end{tabular}




\begin{tabular}{|c|c|c|c|c|}
\hline 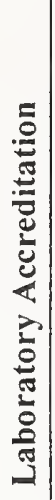 & 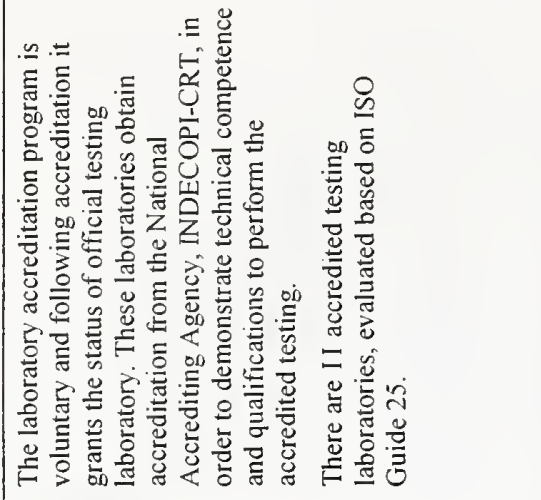 & & 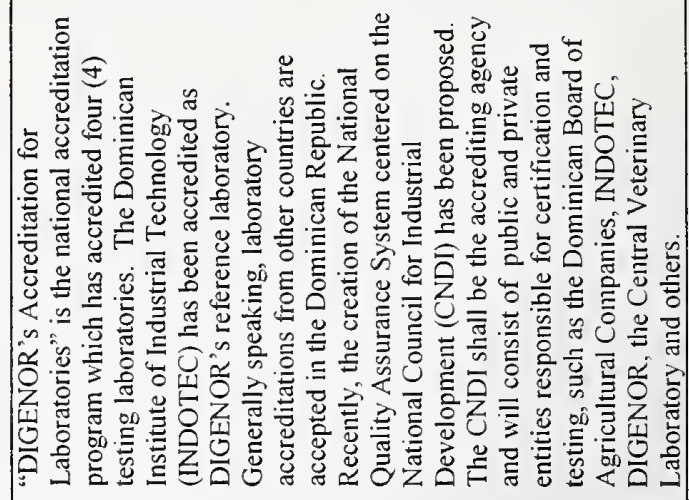 & $\mid$ \\
\hline 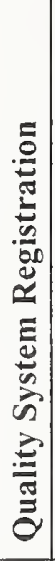 & 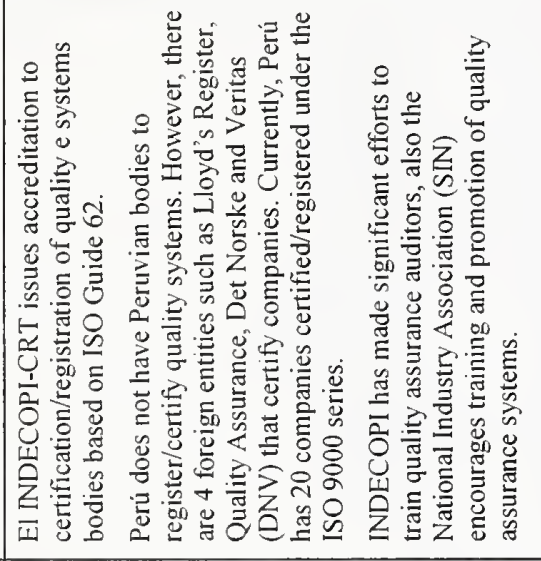 & & 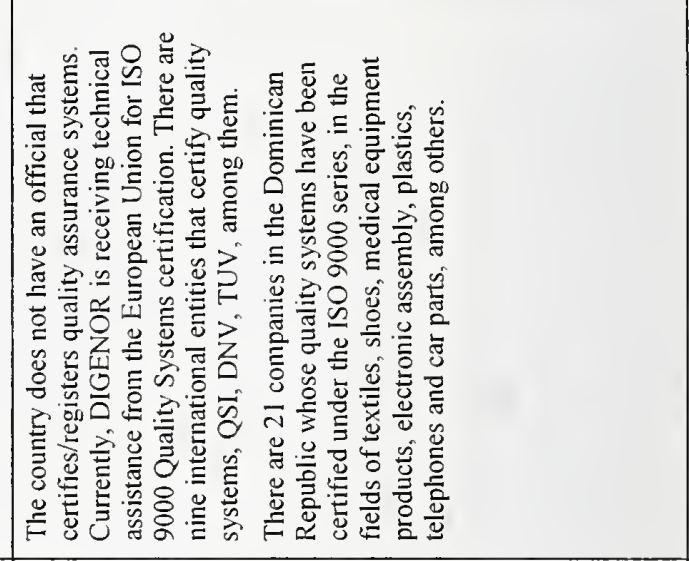 & 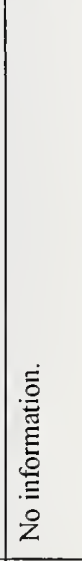 \\
\hline 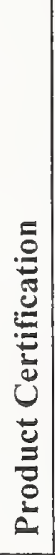 & 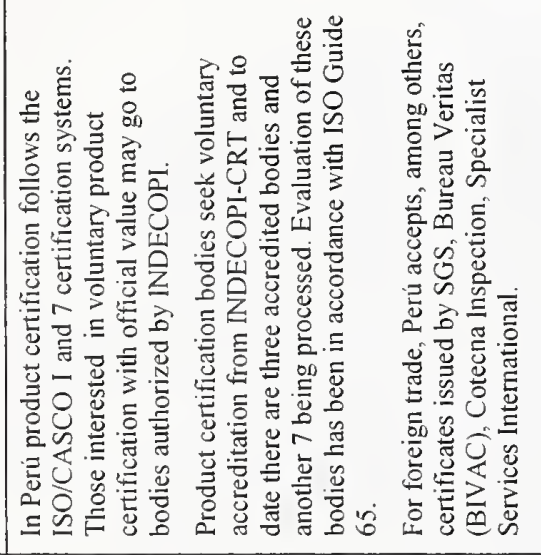 & & 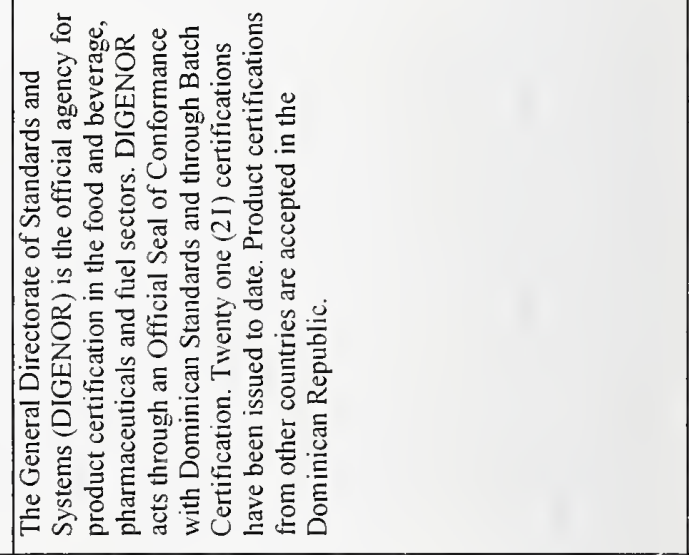 & 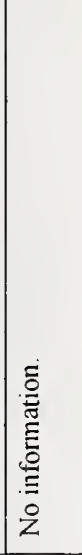 \\
\hline 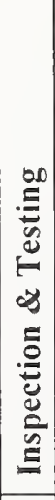 & 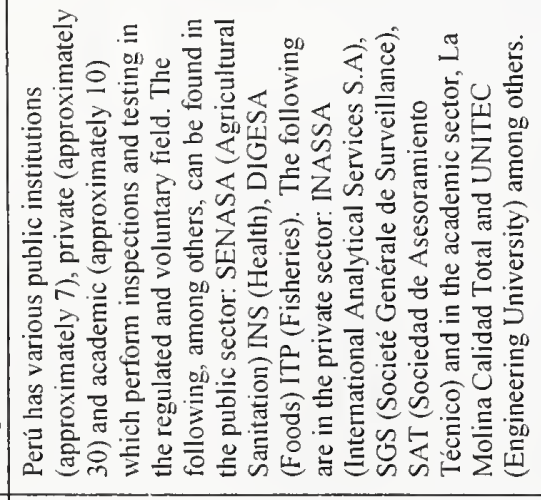 & 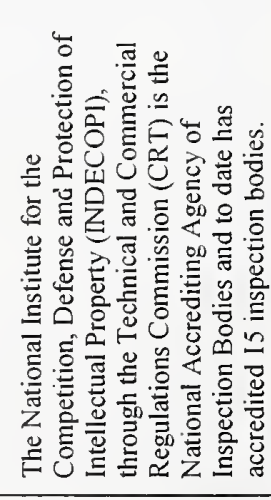 & 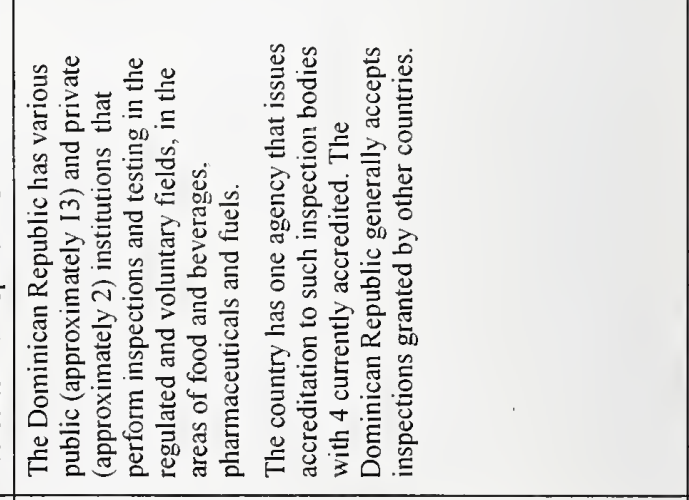 & 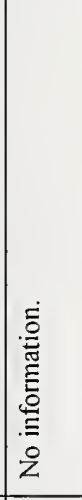 \\
\hline 总 & $\mid \begin{array}{l}\Xi \\
\bar{\Xi} \\
2\end{array}$ & & 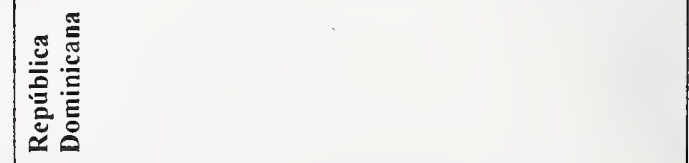 & 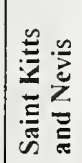 \\
\hline
\end{tabular}




\begin{tabular}{|c|c|c|c|}
\hline & 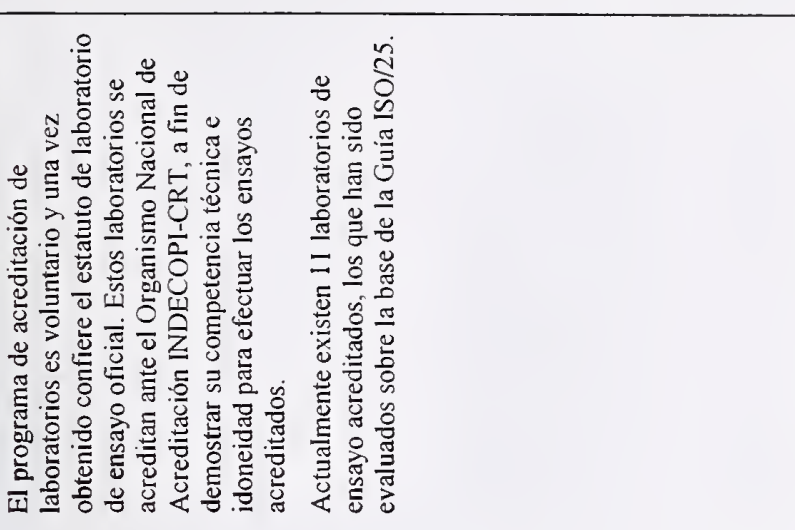 & 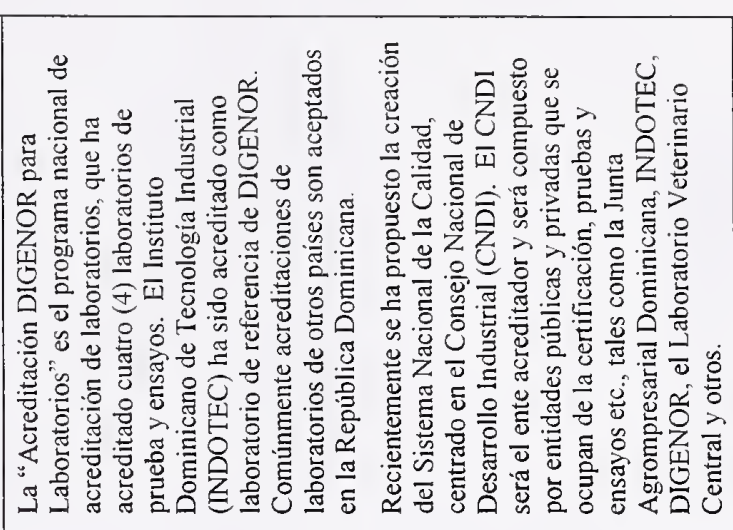 & $\frac{1}{\frac{1}{2}}$ \\
\hline & 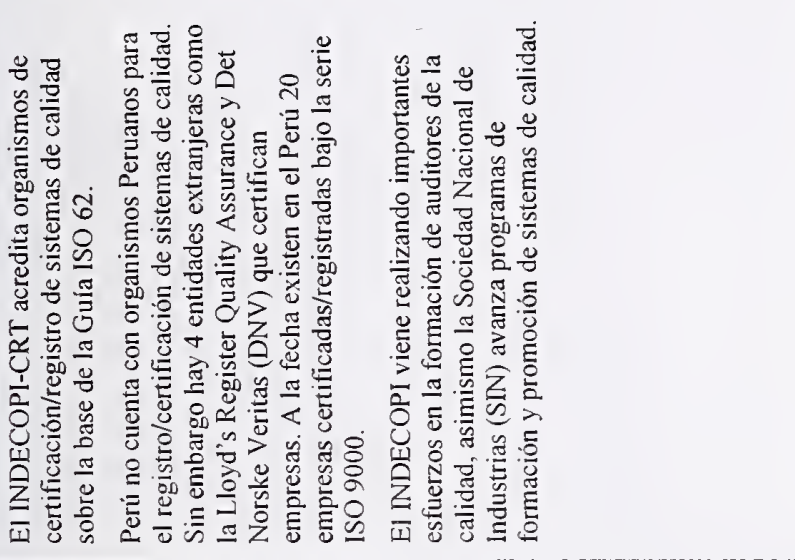 & 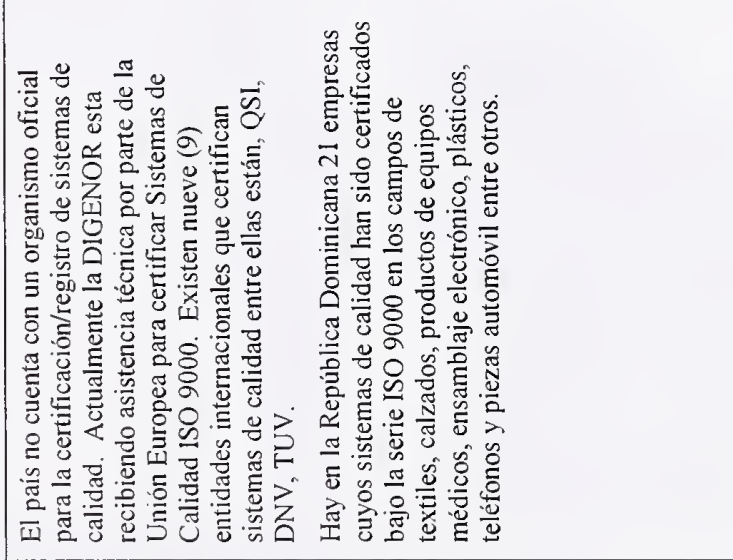 & \\
\hline & 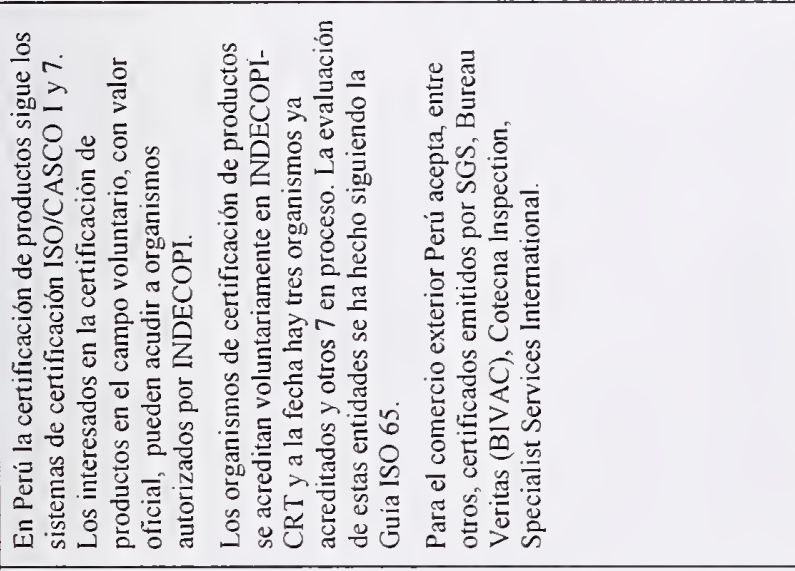 & 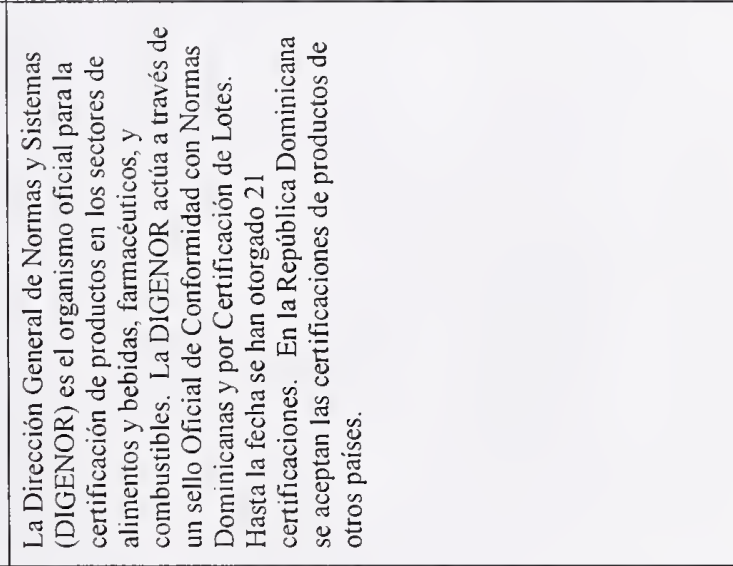 & \\
\hline & 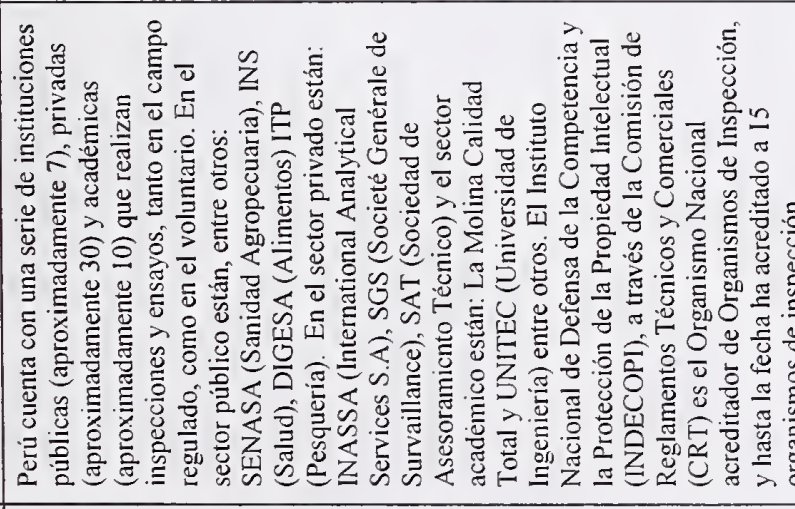 & 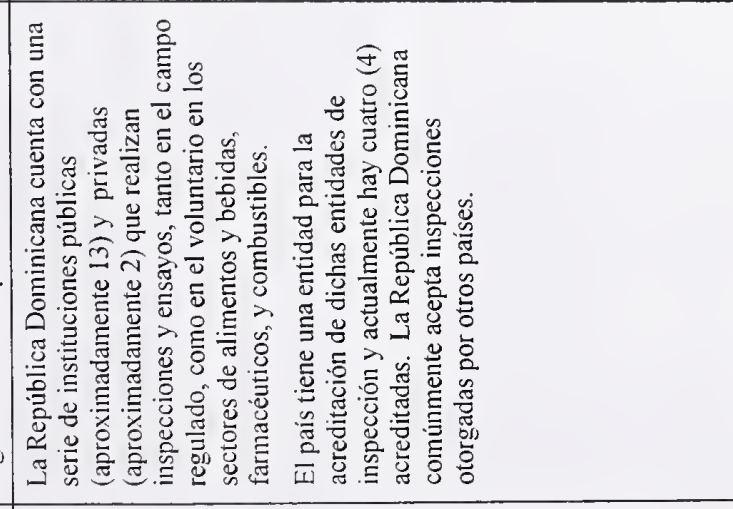 & 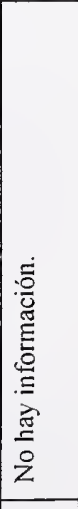 \\
\hline & 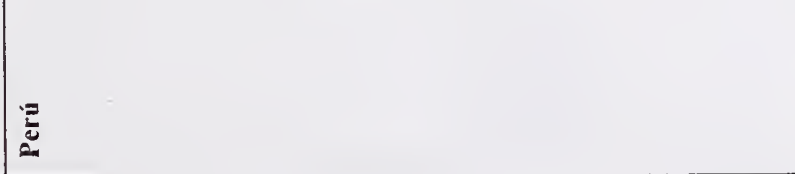 & 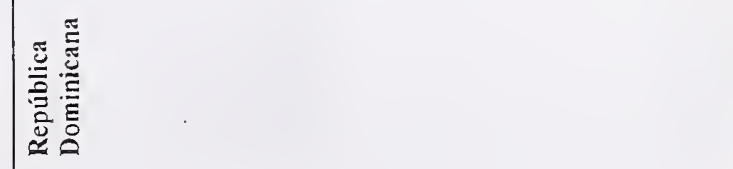 & 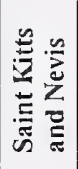 \\
\hline
\end{tabular}




\begin{tabular}{|c|c|c|c|c|}
\hline 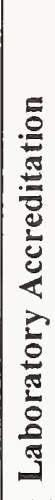 & 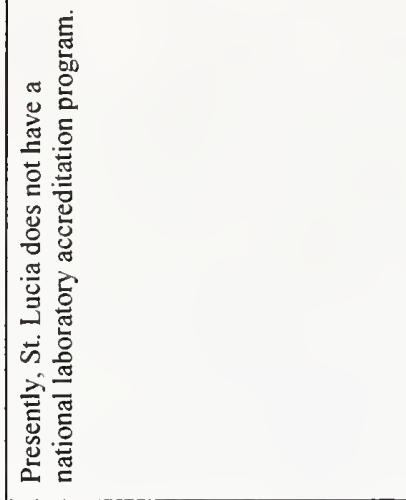 & 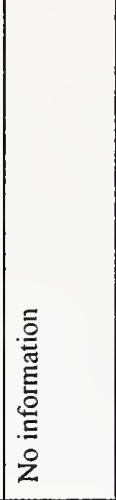 & 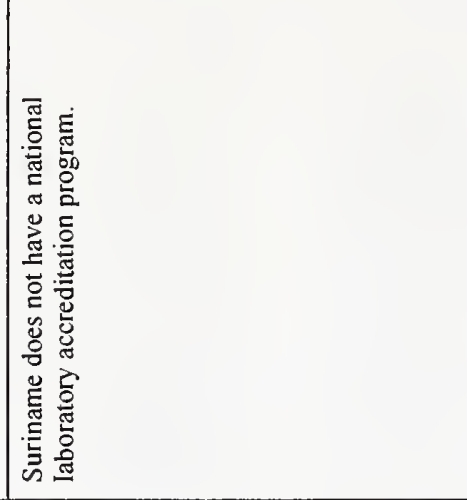 & 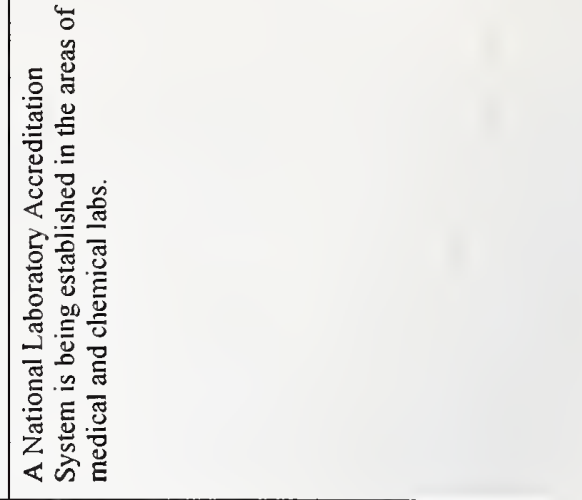 \\
\hline 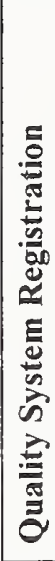 & 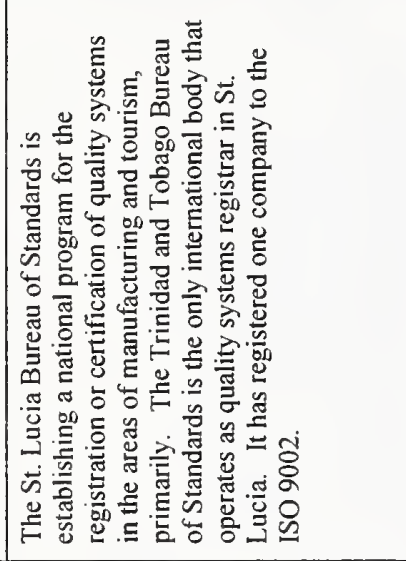 & 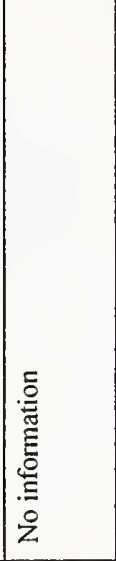 & 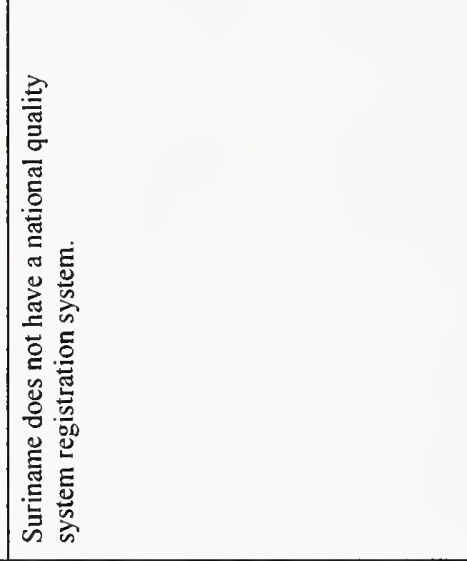 & 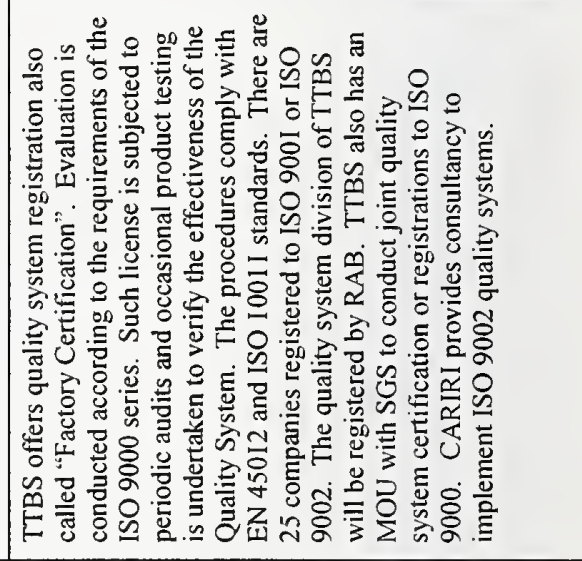 \\
\hline 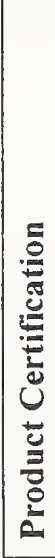 & 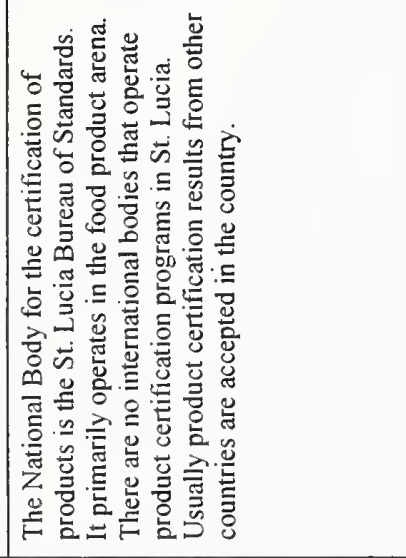 & 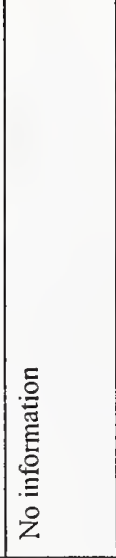 & 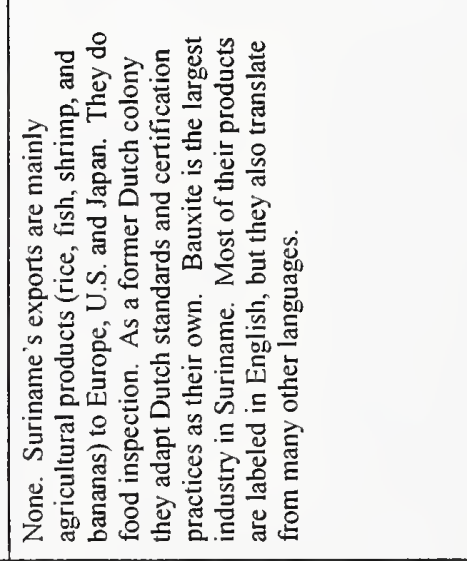 & 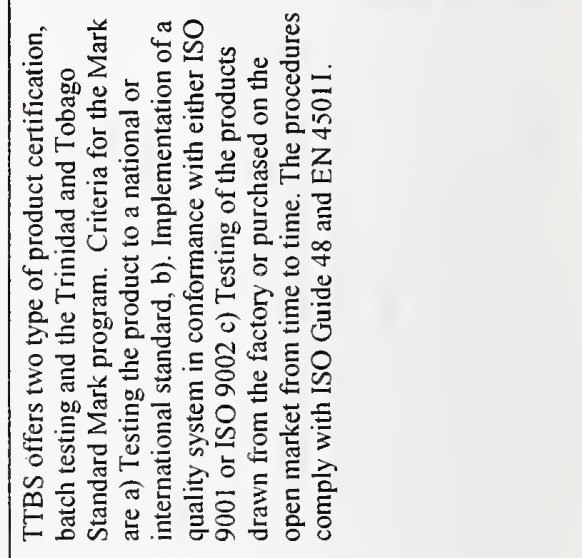 \\
\hline 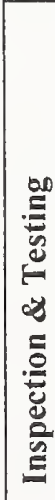 & 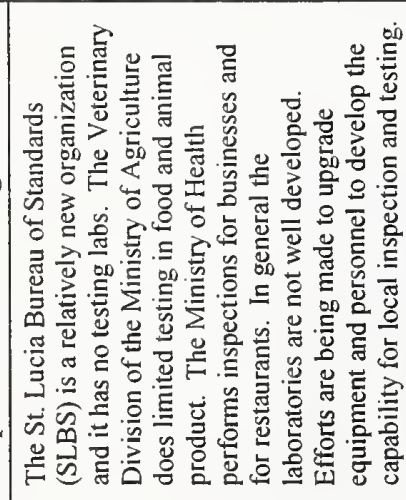 & 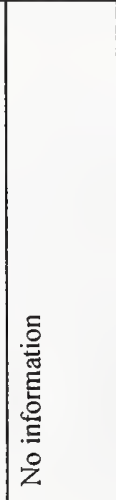 & 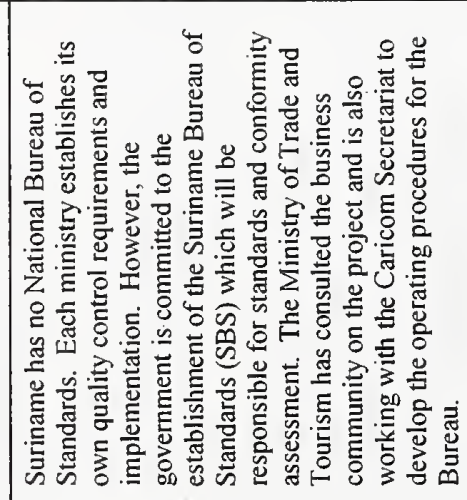 & 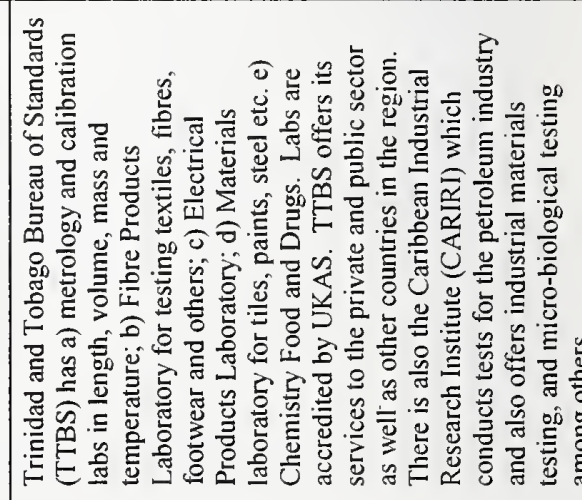 \\
\hline 总 & 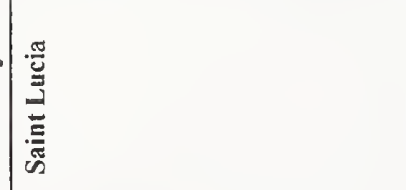 & 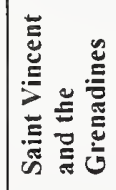 & 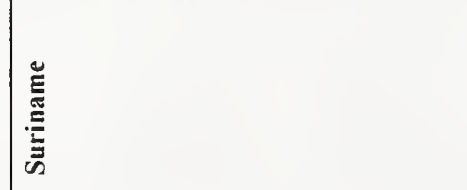 & 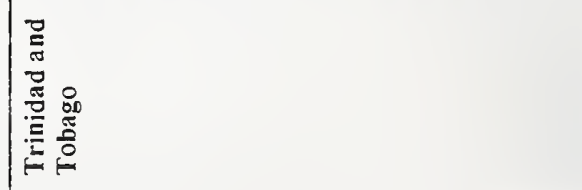 \\
\hline
\end{tabular}




\begin{tabular}{|c|c|c|c|c|}
\hline 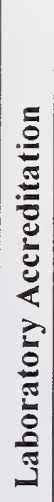 & 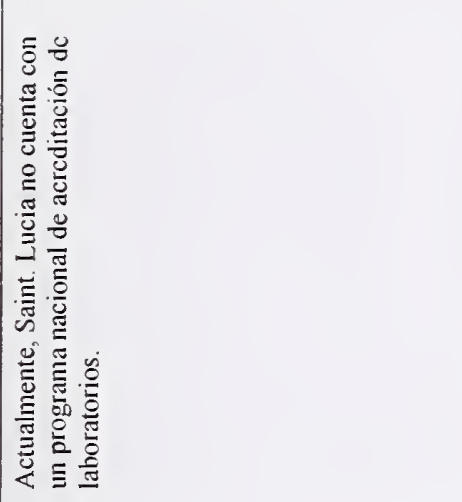 & 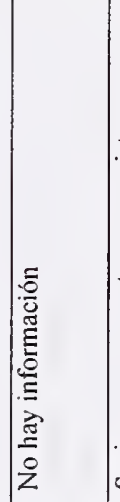 & 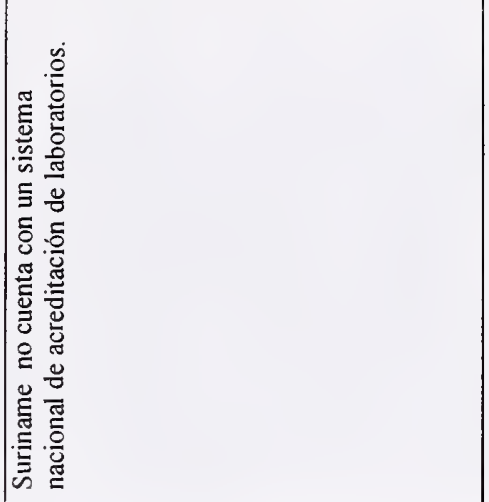 & 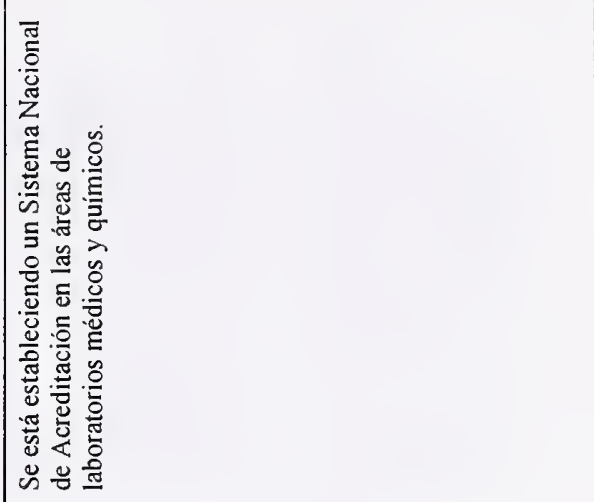 \\
\hline 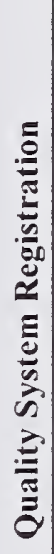 & 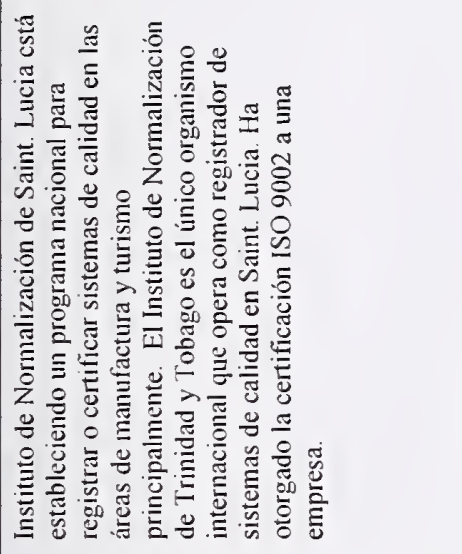 & 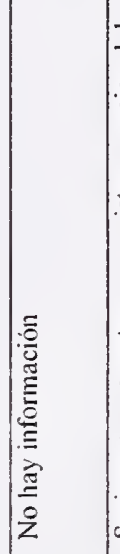 & 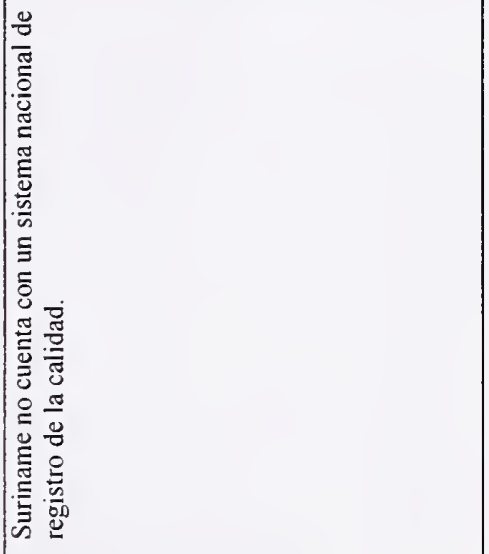 & 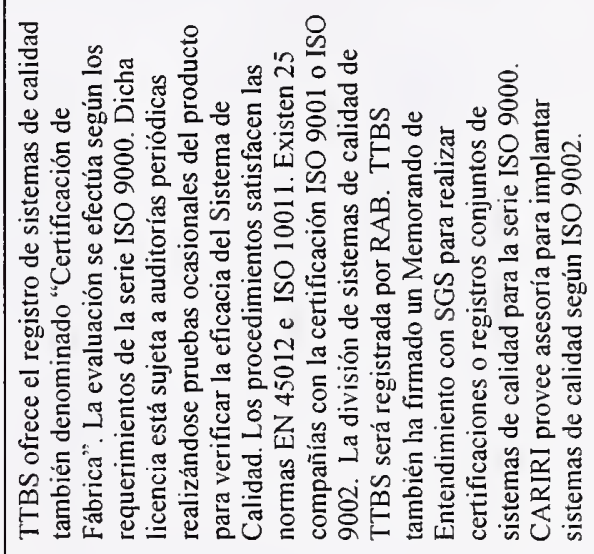 \\
\hline & 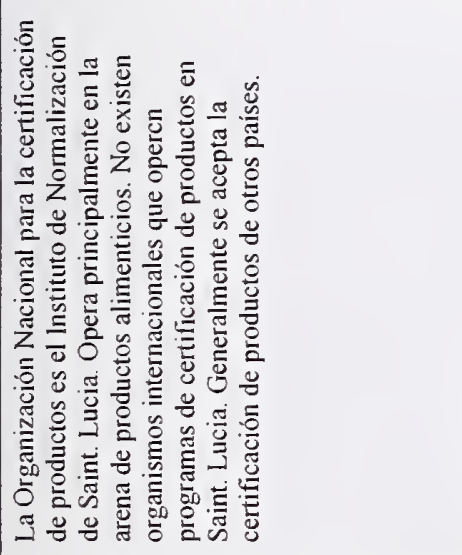 & 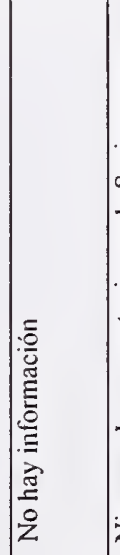 & 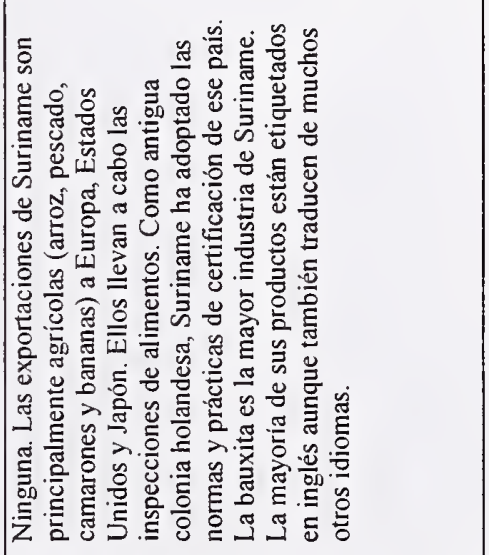 & 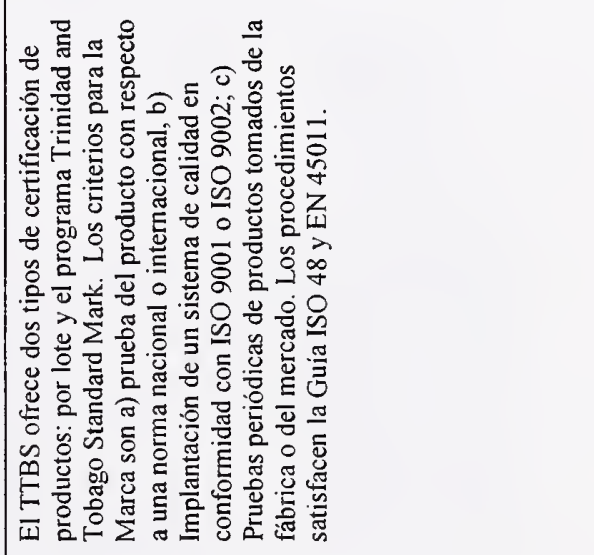 \\
\hline 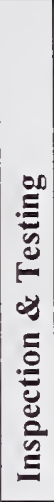 & 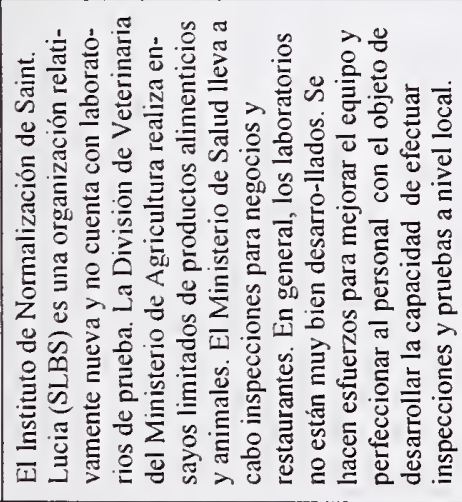 & $\mid$ & 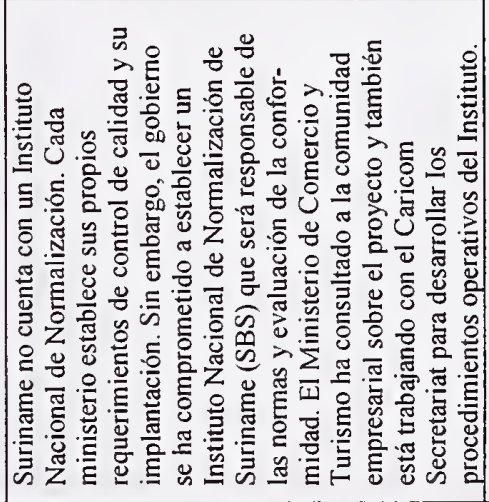 & 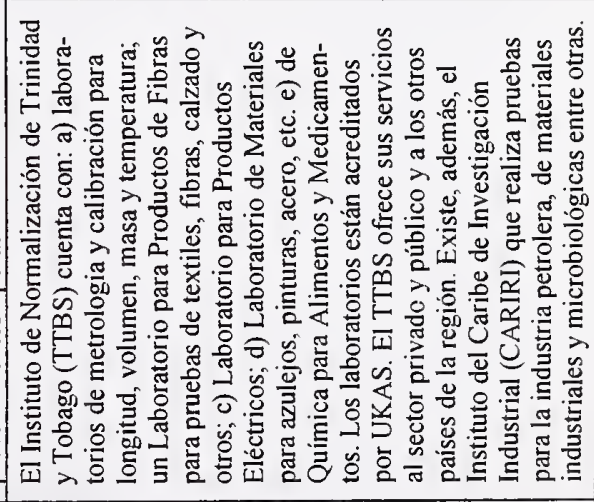 \\
\hline 己̇ & 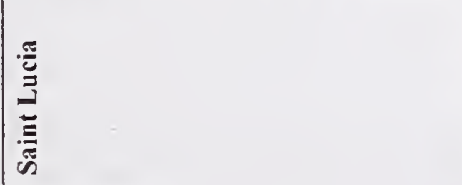 & 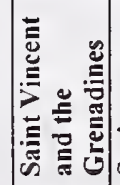 & & 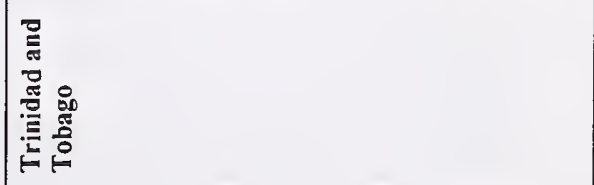 \\
\hline
\end{tabular}




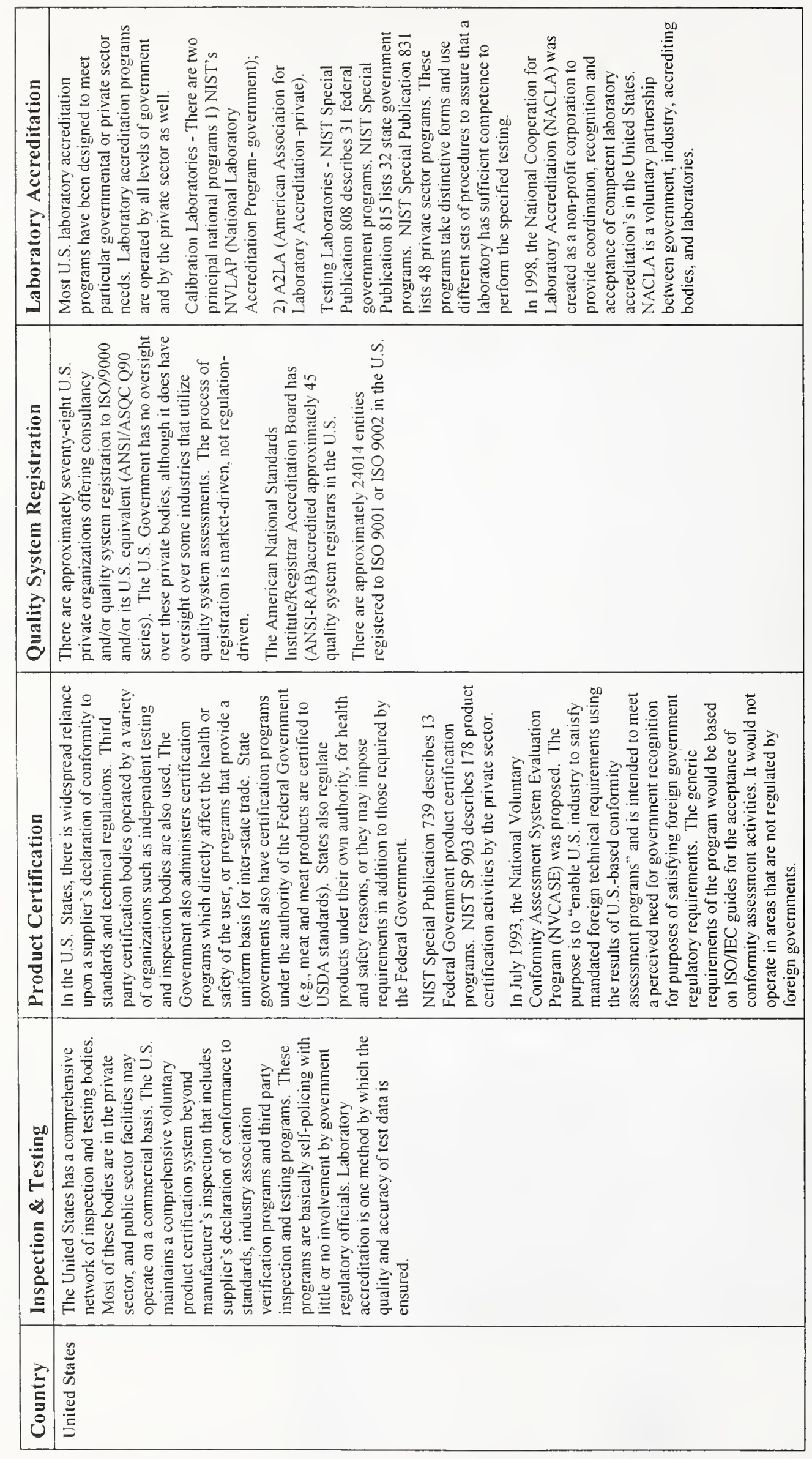




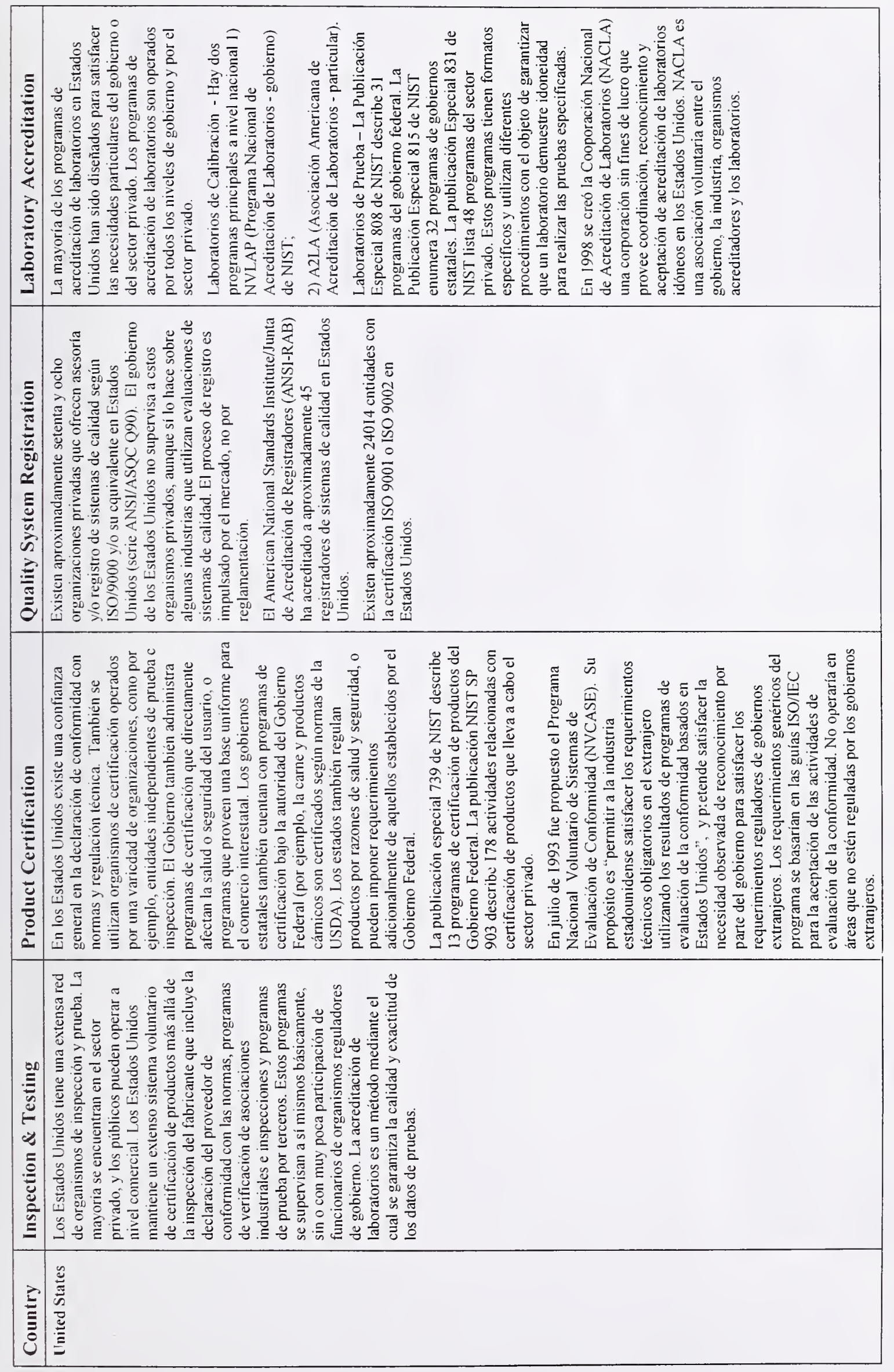




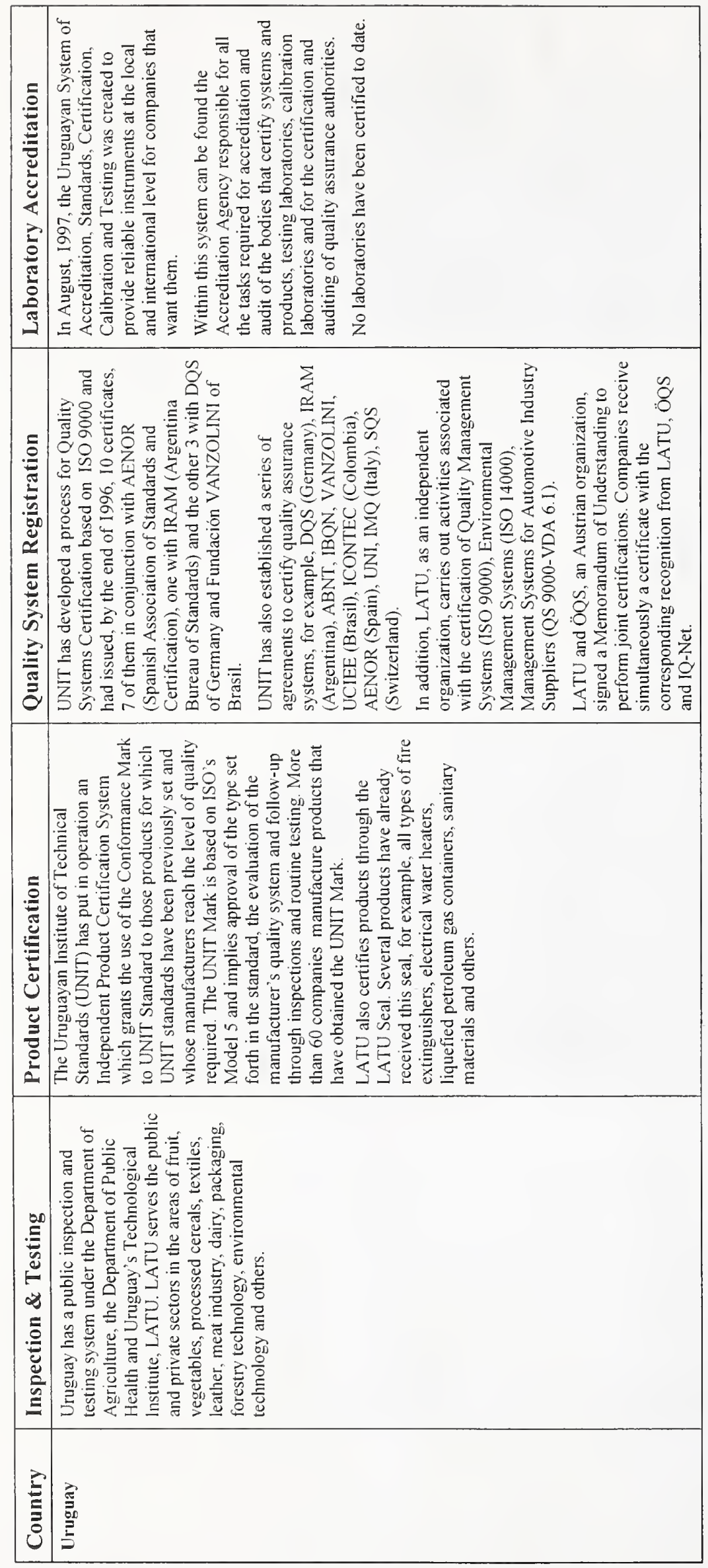




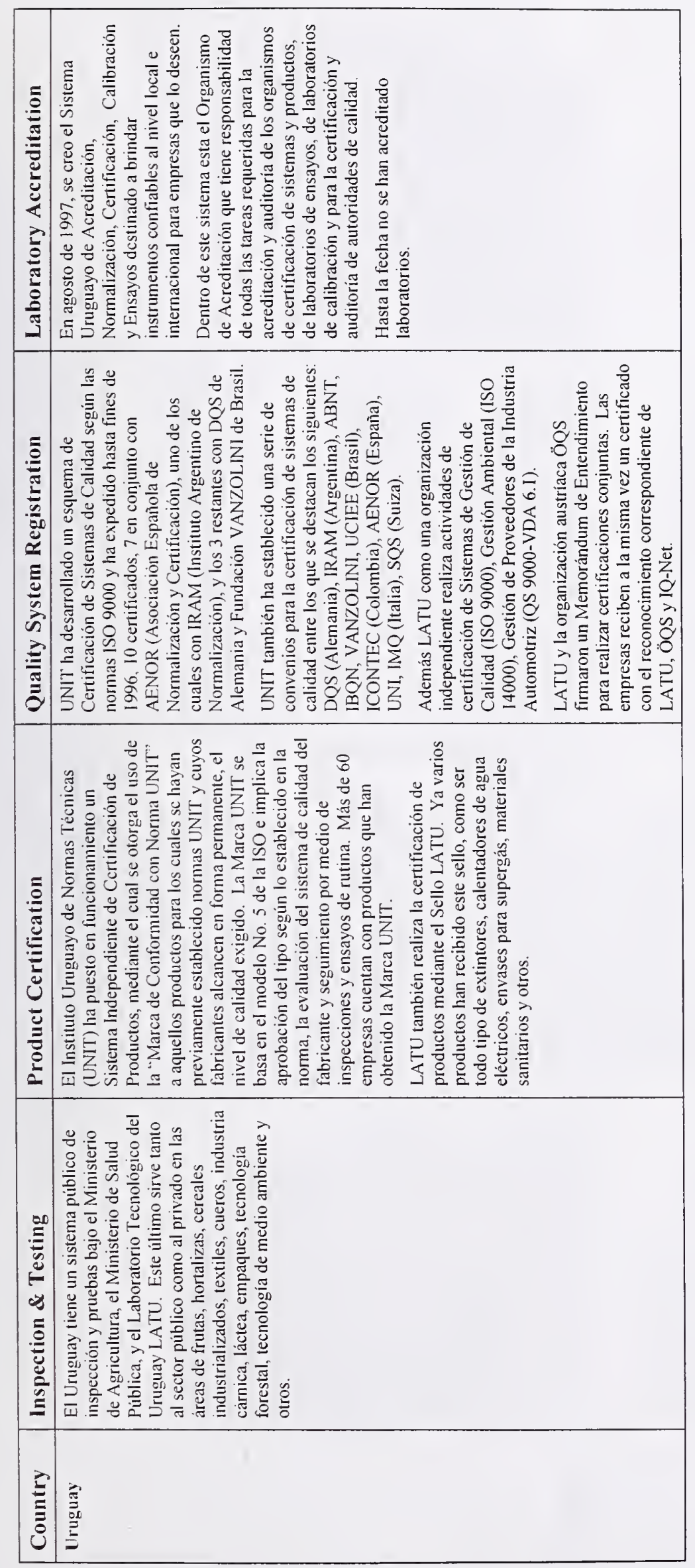




\begin{tabular}{|c|c|c|}
\hline & 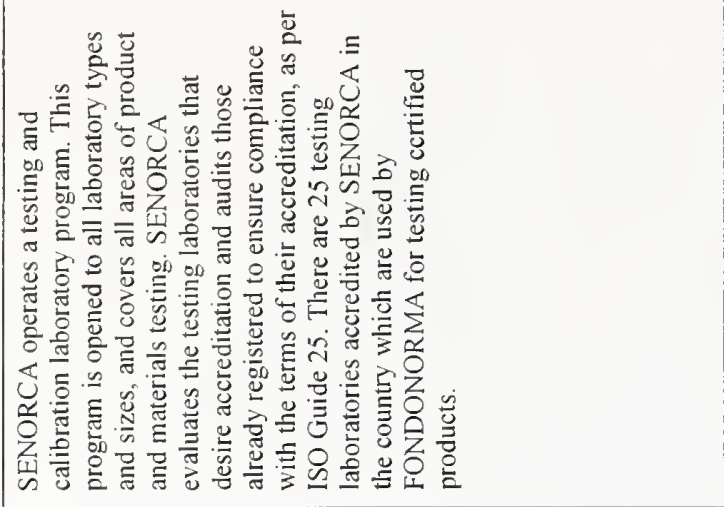 & 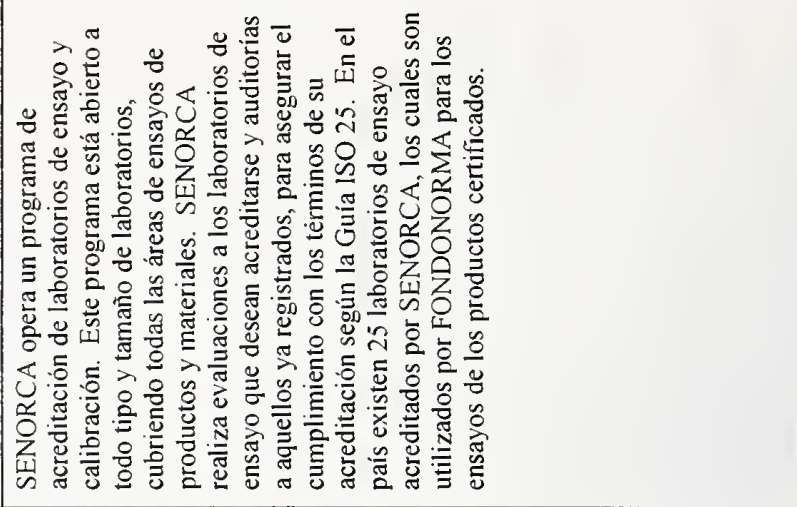 \\
\hline & 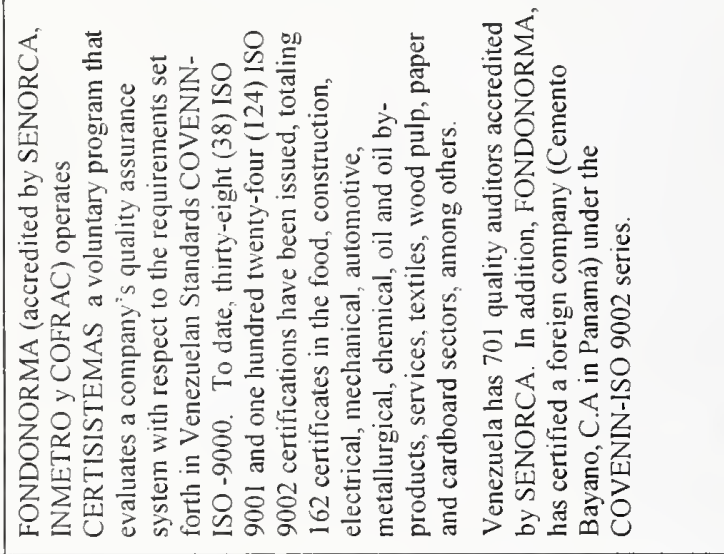 & 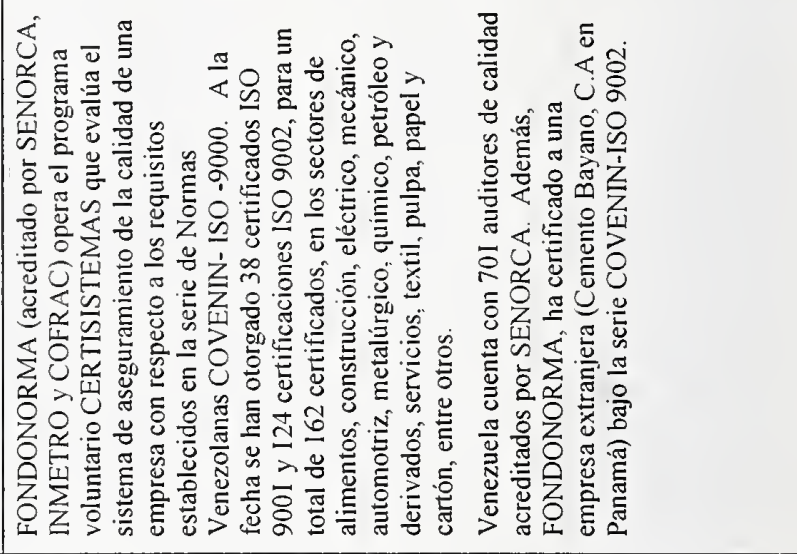 \\
\hline & 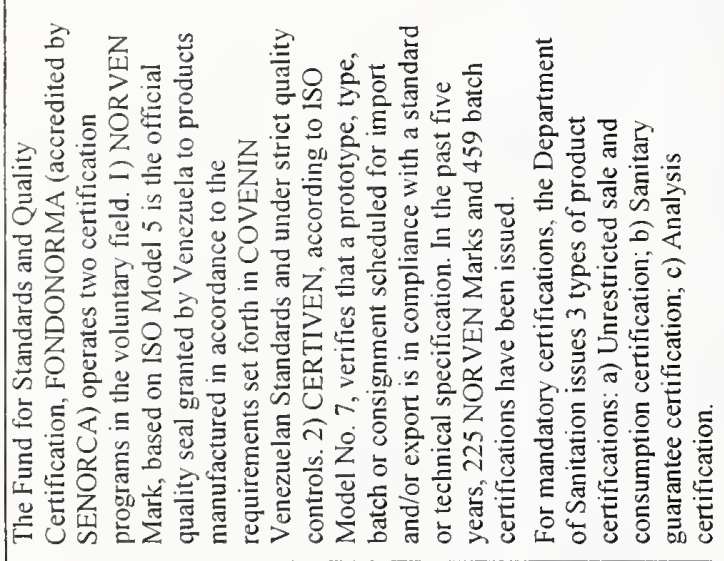 & 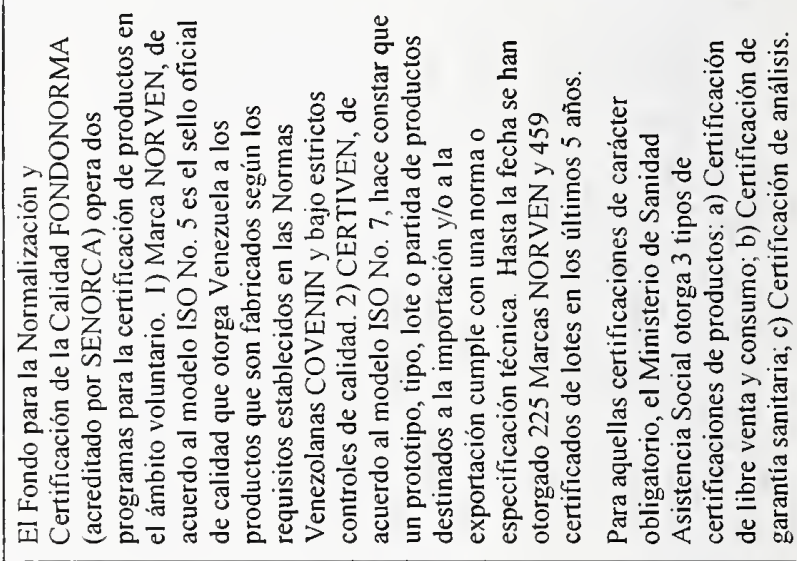 \\
\hline 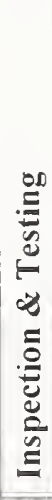 & 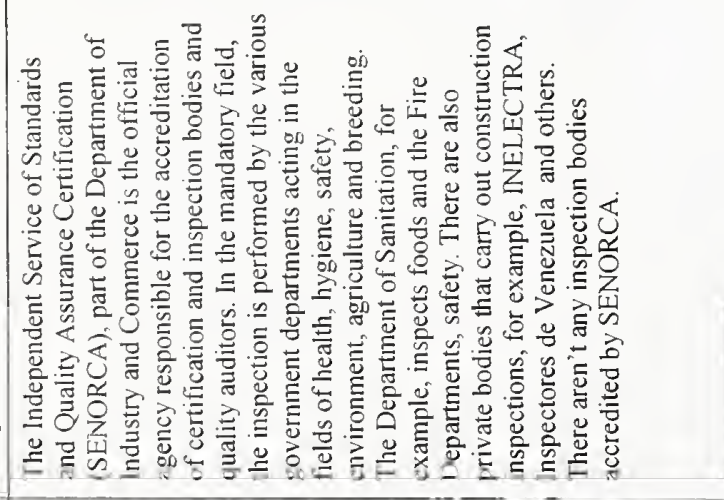 & 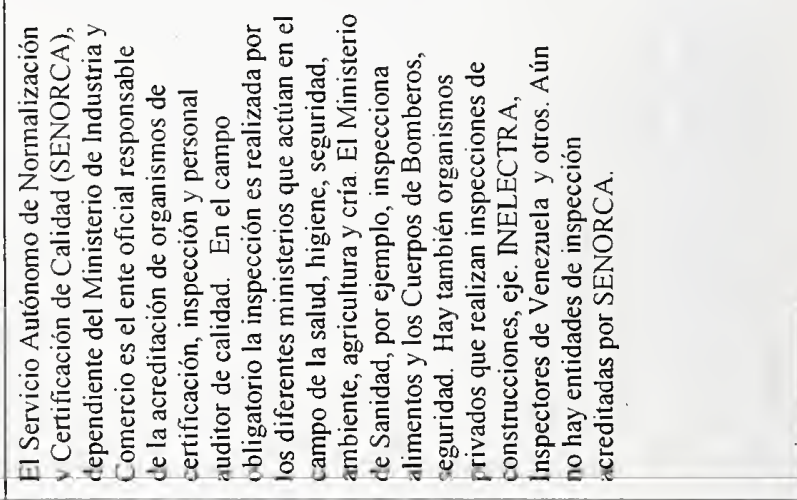 \\
\hline 总 & & \\
\hline
\end{tabular}


Section II: Membership in International Regional Bodies 


\section{Table 1 Explanatory Notes}

Table 1 lists membership in the World Trade Organization (WTO). It also indicates if a country has submitted an implementation statement, established an enquiry point or signed the Code of Good Practice (Annex 3).

\section{Implementation Statement}

Each WTO Member must notify Members of the measures in existence or taken to ensure the implementation and administration of the Agreement and of any subsequent changes to them (Article 15.2). The statement must include:

1. All relevant laws, regulations, administrative orders, and other relevant documentation that ensures that the provisions of the Agreement are being applied;

2. The names of the publications where technical regulations, standards and conformity assessment procedures are published;

3. The expected length of time for the presentation of written comments on technical regulations, standards or conformity assessment procedures;

4. The name and address of the Enquiry Points established under Article 10.

\section{Enquiry Point}

As a complement to the obligation to notify, each WTO Member must set up a national enquiry point. The enquiry point is a focal point where other WTO Members can request and obtain information and documentation on: (a) Member's technical regulations; (b) standards and conformity assessment procedures, whether impending or adopted; (c) participation in bilateral or multilateral standard-related agreements, regional standardizing bodies and conformity assessment systems (Article 10).

Enquiry points can be governmental bodies or be assigned to private agencies. There may be more than one enquiry point per country, but they must coordinate with one another and provide complete information to interested parties. The obligation to set up enquiry points is important for countries wishing to acquire information from other Members on foreign regulations and standards affecting products in which they have a trade interest.

\section{Code of Good Practice}

The Code of Good Practice for the Preparation, Adoption and Application of Standards defines disciplines for central government, local government, non-governmental and regional standards bodies developing voluntary standards. The Code is recommended for adoption by all of these standards bodies; however, central government standards bodies must accept and comply with the provisions of the Code. A standards body wishing to adhere to or withdraw from the Code has to notify its acceptance of or withdrawal from the Code using the appropriate notification. Standards bodies that have accepted the Code must report their work program at least twice a year and where details of this program can be obtained. Notifications have to be sent either directly to the ISO/IEC Information Center in Geneva, or to the national member of ISO/IEC. 
Table 1: WTO Membership and Selected Obligations

\begin{tabular}{|c|c|c|c|c|}
\hline Country & WTO & $\begin{array}{c}\text { Implementation } \\
\text { Statement } \\
(15.2)\end{array}$ & $\begin{array}{l}\text { Enquiry } \\
\text { Point }\end{array}$ & $\begin{array}{c}\text { Code of Good } \\
\text { Practice } \\
\text { Annex } 3\end{array}$ \\
\hline Antigua \&Barbuda & $\bullet$ & & $\bullet$ & \\
\hline Argentina & - & - & - & - \\
\hline \multicolumn{5}{|l|}{ The Bahamas } \\
\hline Barbados & - & - & - & - \\
\hline Belize & - & & 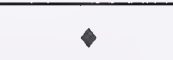 & \\
\hline Bolivia & - & - & - & \\
\hline Brasil & - & - & - & - \\
\hline Canada & - & - & - & \\
\hline Chile & - & - & 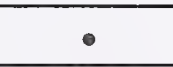 & - \\
\hline Colombia & - & - & - & - \\
\hline Costa Rica & 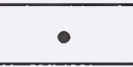 & & - & - \\
\hline Dominica & - & & 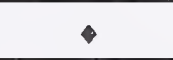 & \\
\hline Ecuador & - & & - & - \\
\hline EI Salvador & - & & $\bullet$ & - \\
\hline Grenada & - & & 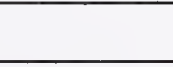 & $\bullet$ \\
\hline Guatemala & $\cdot$ & & $\checkmark$ & \\
\hline Guyana & - & & 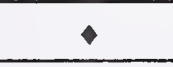 & - \\
\hline Häiti & $\bullet$ & & & \\
\hline Honduras & - & - & - & \\
\hline Jamaica & $\bullet$ & 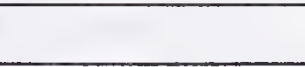 & $\bullet$ & - \\
\hline México & - & - & - & - \\
\hline Nicaragua & - & & 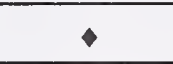 & \\
\hline Panamá & - & - & $\bullet$ & 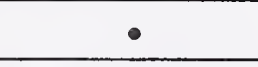 \\
\hline Paraguay & - & & 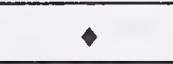 & \\
\hline Perú & $\cdot$ & $\bullet$ & $\bullet$ & $\bullet$ \\
\hline República Dominicana & - & & $\bullet$ & - \\
\hline St. Kitts \& Nevis & $\bullet$ & & & \\
\hline Saint Lucia & - & - & - & \\
\hline St. Vincent \& Grenadines & - & & & \\
\hline Suriname & - & & & \\
\hline Trinidad \&Tobago & $\bullet$ & $\bullet$ & - & - \\
\hline United States & - & - & $\cdot$ & - \\
\hline Uruguay & - & & - & $\bullet$ \\
\hline Venezuela & - & & 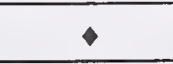 & - \\
\hline
\end{tabular}


1. Column 2 lists the acronyms for the National Standards Bodies (NSB) of the FTAA countries. The full name and address are included in Section III.

2. The United States does not have an official NSB. The American National Standards Institute (ANSI) is a federation of private sector standards developers and is the U.S. representative to the ISO. Not all the standards developers in the United States belong to ANSI, nor are they obligated to do so.

3. The International Organization for Standardization (ISO) is a private international organization dedicated to voluntary standardization. Its membership consists of recognized national standards bodies from 129 countries. Membership in ISO is by the National Standards Body listed in column 2.

4. The ISO Information Network (ISONET) links the information centers of the ISO members into a coherent information system. ISONET is an agreement between standards bodies to combine their efforts in order to make information on standards, technical regulations and related matters readily available whenever it is required. Column 5 lists the ISO members that are members of ISONET.

5. The Panamerican Standards Commission (COPANT) is a regional standards organization that develops or harmonizes regional standards where appropriate and coordinates regional positions and representation to the ISO. Membership in COPANT is by the National Standards Body listed in column 2.

\section{Table 3 Explanatory Notes}

1. Unlike the ISO and COPANT, membership in ITU or IEC is not necessarily by the National Standards Body listed in Table 2. The International Telecommunications Union (ITU) is a treaty organization that develops standards in the telecommunication and services industries. Membership in ITU is comprised of government representatives from 185 countries. In the United States, the Department of State coordinates representation.

2. The International Electrotechnical Commission (IEC) develops standards for electrical and electronic engineering products and devices. Membership in IEC is by the presidents of the national committees of 60 countries. The U.S. National Committee to the IEC is housed at ANSI.

3. Codex Alimentarius Commission (Food Code in Latin) is a subsidiary of the Food and Agriculture Organization of the United Nations (FAO) and the World Health Organization (WHO). The Codex system was set up to facilitate trade in food while protecting consumer's health and ensuring fair practices in food trade. Codex is the compilation of all the Standards, Codes of Practice, Guidelines and Recommendations of the Codes Alimentarius Commission and of government reactions to these. To be comprehensive, Codex was included in this document since many FTTA countries have economies which are largely based on the export of food products. 
Table 2: Membership to International and Regional Standards Bodies

\begin{tabular}{|c|c|c|c|c|c|}
\hline \multirow[t]{2}{*}{ Country } & \multirow{2}{*}{$\begin{array}{c}\text { National } \\
\text { Standards Body }\end{array}$} & \multirow[t]{2}{*}{ Type } & \multicolumn{3}{|c|}{ Membership } \\
\hline & & & ISO & ISONET & COPANT \\
\hline Antigua \& Barbuda & $\mathrm{ABBS}$ & Gov & & & \\
\hline Argentina & IRAM & Prv & - & - & - \\
\hline The Bahamas & $\begin{array}{l}\text { Agriculture Dept. } \\
\text { Env. Health Dept. }\end{array}$ & Gov & & & \\
\hline Barbados & BNSI & Mix & $\bullet(\mathrm{C})$ & - & - \\
\hline Belize & BBS & & & & \\
\hline Bolivia & IBNORCA & Prv & $\bullet(\mathrm{C})$ & & - \\
\hline Brasil & ABNT & Prv & - & - & - \\
\hline Canada & $\mathrm{SCC}$ & Mix & - & - & - \\
\hline Chile & INN & Prv & - & - & - \\
\hline Colombia & ICONTEC & Prv & - & - & - \\
\hline Costa Rica & INTECO & Mix & - & - & - \\
\hline Dominica & None & & & & \\
\hline Ecuador & INEN & Gov & - & - & \\
\hline El Salvador & CONACYT & Gov & $\bullet(\mathrm{C})$ & & - \\
\hline Grenada & GDBS & Gov & $\bullet(\mathrm{S})$ & & - \\
\hline Guatemala & COGUANOR & Mix & $\bullet(\mathrm{C})$ & & - \\
\hline Guyana & GNBS & Gov & $\bullet(\mathrm{S})$ & & - \\
\hline Häiti & None & & & & \\
\hline Hond uras & DNM-SIC & Gov & & & \\
\hline Jamaica & JBS & Gov & - & - & - \\
\hline México & DGN & Gov & $\bullet$ & $\bullet$ & - \\
\hline Nicaragua & DGCT & Gov & $\bullet(\mathrm{C})$ & & \\
\hline Panamá & DGNTI & Gov & - & - & - \\
\hline Paraguay & INTN & Gov & $\cdot(\mathrm{C})$ & & - \\
\hline Perú & INDECOPI & Gov & $\bullet(\mathrm{C})$ & & - \\
\hline República Dominicana & DIGENOR & Gov & $\bullet(S)$ & & - \\
\hline Saint Lucia & SLBS & Gov & $\bullet(S)$ & & \\
\hline St Kitts \& Nevis & ni & & & & \\
\hline St Vincent \& Grenadines & ni & & & & \\
\hline Suriname & None & & & & \\
\hline Trinidad \&Tobago & TTBS & Mix & - & $\bullet$ & - \\
\hline United States & ANSI* & Prv & - & - & - \\
\hline Uruguay & UNIT & Prv & - & & $\cdot$ \\
\hline Venezuela & FONDONORMA & Prv & - & - & $\bullet$ \\
\hline
\end{tabular}


Table 3: Membership to other International Standards Bodies

\begin{tabular}{|l|c|c|c|}
\hline \multicolumn{1}{|c|}{ Country } & \multicolumn{3}{c|}{ Membership } \\
\cline { 2 - 4 } & ITU & IEC & CODEX \\
\hline Antigua \& Barbuda & & & $\bullet$ \\
\hline Argentina & $\bullet$ & & $\bullet$ \\
\hline The Bahamas & $\bullet$ & & \\
\hline Barbados & $\bullet$ & & $\bullet$ \\
\hline Belize & $\bullet$ & & $\bullet$ \\
\hline Bolivia & $\bullet$ & & $\bullet$ \\
\hline Brasil & $\bullet$ & $\bullet$ & $\bullet$ \\
\hline Canada & $\bullet$ & $\bullet$ & $\bullet$ \\
\hline Chile & $\bullet$ & & $\bullet$ \\
\hline Colombia & $\bullet$ & $\bullet(P)$ & $\bullet$ \\
\hline Costa Rica & $\bullet$ & & $\bullet$ \\
\hline Dominica & $\bullet$ & & $\bullet$ \\
\hline Ecuador & $\bullet$ & & $\bullet$ \\
\hline El Salvador & $\bullet$ & & $\bullet$ \\
\hline Grenada & $\bullet$ & & $\bullet$ \\
\hline Guatemala & $\bullet$ & & $\bullet$ \\
\hline Guyana & $\bullet$ & & $\bullet$ \\
\hline Häiti & $\bullet$ & & $\bullet$ \\
\hline Honduras & $\bullet$ & & $\bullet$ \\
\hline Jamaica & $\bullet$ & & $\bullet$ \\
\hline México & $\bullet$ & & $\bullet$ \\
\hline Nicaragua & $\bullet$ & & $\bullet$ \\
\hline Panamá & $\bullet$ & & $\bullet$ \\
\hline Paraguay & $\bullet$ & & $\bullet$ \\
\hline Perú & $\bullet$ & & $\bullet$ \\
\hline República Dominicana & $\bullet$ & & $\bullet$ \\
\hline Saint Lucia & $\bullet$ & & $\bullet$ \\
\hline St Kitts \& Nevis & $\bullet$ & & $\bullet$ \\
\hline St Vincent \& Grenadines & & & $\bullet$ \\
\hline Suriname & $\bullet$ & & $\bullet$ \\
\hline Trinidad \&Tobago & $\bullet$ & & $\bullet$ \\
\hline United States & $\bullet$ & & $\bullet$ \\
\hline Uruguay & $\bullet$ & & $\bullet$ \\
\hline Venezuela & $\bullet$ & & $\bullet$ \\
\hline
\end{tabular}

$(\mathrm{P})=$ Pre-associate Member $\bullet=$ Yes $\quad$ Blank $=$ No 
Table 4: National Metrology Laboratories

\begin{tabular}{|c|c|c|}
\hline Country & National Metrolc & Laboratory \\
\hline Antigua \&Barbuda & ABBS & Antigua \& Barbuda Bureau of Standards \\
\hline Argentina & INTI & Instituto Nacional de Tecnología Industrial \\
\hline The Bahamas & & Ministry of Consumer Welfare \\
\hline Barbados & BNSI & Barbados National Standards Institution \\
\hline Belize & BBS & Belize Bureau of Standards \\
\hline Bolivia & IBMETRO & Instituto Boliviano de Metrología \\
\hline Brasil & INMETRO & Instituto Nacional de Metrología, Normalización y Calidad Industrial \\
\hline Canada & NRC & National Research Council \\
\hline Chile & & National Metrology Network coordinated by INN \\
\hline Colombia & & Centro Nacional de Control de Calidad y Metrología \\
\hline Costa Rica & ONNUM & Oficina Nacional de Normas y Unidades de Medida \\
\hline Dominica & & Ministry of Trade and Market \\
\hline Ecuador & INEN & Instituto Ecuatoriano de Normalización \\
\hline El Salvador & CONACYT & Consejo Nacional de Ciencia y Tecnología \\
\hline Grenada & GDBS & Grenada Bureau of Standards \\
\hline Guatemala & COGUANOR & Comisión Guatemalteca de Normas \\
\hline Guyana & GNBS & Guyana National Bureau of Standards \\
\hline Häiti & & Ministry of Commerce and Industry \\
\hline Honduras & & $\begin{array}{l}\text { Depto. de Normalización y Metrología de la SIC-Instituto de } \\
\text { Metrología y Calidad Consejo Hondureño de Ciencia y Tecnología }\end{array}$ \\
\hline Jamaica & JBS & Jamaica Bureau of Standards \\
\hline México & CENAM & Centro Nacional de Metrología \\
\hline Nicaragua & LABML & $\begin{array}{l}\text { Ministerio de Fomento, Industria y Comercio } \\
\text { Dirección de Normalización y Metrología }\end{array}$ \\
\hline Panamá & DGNTI & Dirección General de Normas y Tecnología Industrial \\
\hline Paraguay & INTN & Instituto Nacional de Tecnología y Normalización \\
\hline Perú & SNM-INDECOPI & Servicio Nacional de Metrología del INDECOPI \\
\hline República Dominicana & DIGENOR & Dirección General de Normas y Sistemas de Calidad \\
\hline St Kitts \& Nevis & & Ministry of Development \\
\hline Saint Lucia & SLBS & St. Lucia Bureau of Standards \\
\hline St Vincent \& Grenadines & & Government of Saint Vincent \& Grenadines \\
\hline Suriname & & Metrology Unit - Department of Trade \& Industry \\
\hline Trinidad \& Tobago & TTBS & Trinidad \&Tobago Bureau of Standards \\
\hline United States & NIST & National Institute of Standards and Technology \\
\hline Uruguay & LATU & Sector de Metrología, Laboratorio Tecnológico del Uruguay \\
\hline Venezuela & SANAMET & Servicio Autónomo Nacional de Metrología \\
\hline
\end{tabular}




\section{$\underline{\text { Table } 5 \text { Explanatory Notes }}$}

1. The International Organization of Legal Metrology (OIML) is an intergovernmental treaty organization whose membership includes Member States (countries which participate actively in technical activities) and Corresponding Members (countries which join OIML as observers). OIML promotes global harmonization of legal metrology procedures and provides metrological guidelines for the elaboration of national and regional requirements concerning the manufacture and use of measuring instruments for legal metrology applications.

2. The Bureau International des Poids et Mesures (BIPM) (International Bureau of Weights and Measures) ensures world-wide uniformity of measurements and their traceability to the International System of Units. It does this with the authority of the Convention of the Metre, a diplomatic treaty among forty-eight nations. It operates through a series of Consultative Committees whose members are the national metrology laboratories of the nations that have signed the treaty.

3. The Interamerican Metrology System (SIM) brings together the National Metrology Laboratories of the FTAA countries. The goal of SIM is to promote international and regional cooperation throughout the Americas, in order to contribute to the improvement of activities in the domain of scientific, industrial and legal metrology. The region is divided into five subregions, which are Noramet, Camet, Carimet, Andimet, and Suramet. Column 4 lists the relevant subregion for each country. 
Table 5: Metrology Rellated Activities

\begin{tabular}{|c|c|c|c|c|}
\hline Country & OIML & BIPM & SIM & Regional \\
\hline Antigua \& Barbuda & & & $\bullet$ & Carimet \\
\hline Argentina & $\bullet(C)$ & - & - & Suramet \\
\hline The Bahamas & & & - & Carimet \\
\hline Barbados & $\bullet(\mathrm{C})$ & & $\bullet$ & Carimet \\
\hline Belize & & & $\bullet$ & Carimet \\
\hline Bolivia & & & • & Andimet \\
\hline Brasil & - & $\bullet$ & - & Suramet \\
\hline Canada & $\bullet$ & $\bullet$ & $\bullet$ & Noramet \\
\hline Chile & & - & - & Suramet \\
\hline Colombia & $\bullet(\mathrm{C})$ & & $\bullet$ & Andimet \\
\hline Costa Rica & $\bullet(\mathrm{C})$ & & - & Camet \\
\hline Dominica & & & $\bullet$ & Carimet \\
\hline Ecuador & $\bullet(\mathrm{C})$ & & - & Andimet \\
\hline El Salvador & & & 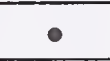 & Camet \\
\hline Grenada & & & $\bullet$ & Carimet \\
\hline Guatemala & $\bullet(C)$ & & $\circ$ & Camet \\
\hline Guyana & & & $\bullet$ & Carimet \\
\hline Häiti & & & $\bullet$ & Carimet \\
\hline Honduras & & & $\bullet$ & Camet \\
\hline Jamaica & & & $\bullet$ & Carimet \\
\hline México & $\bullet(C)$ & $\bullet$ & $\bullet$ & Noramet \\
\hline Nicaragua & & & - & Camet \\
\hline Panamá & $\bullet(C)$ & & $\bullet$ & Camet \\
\hline Paraguay & $\bullet(\mathrm{C})$ & & - & Suramet \\
\hline Perú & $\bullet(\mathrm{C})$ & & $\bullet$ & Andimet \\
\hline República Dominicana & & - & $\bullet$ & Carimet \\
\hline St Kitts \& Nevis & & & $\bullet$ & Carimet \\
\hline Saint Lucia & & & $\bullet$ & Carimet \\
\hline St Vincent \& Grenadines & & & $\bullet$ & Carimet \\
\hline Suriname & & & - & Carimet \\
\hline Trinidad \&Tobago & $\bullet(\mathrm{C})$ & & $\bullet$ & Carimet \\
\hline United States & $\bullet$ & $\circ$ & $\bullet$ & Noramet \\
\hline Uruguay & $\bullet(\mathrm{C})$ & $\bullet$ & $\bullet$ & Suramet \\
\hline Venezuela & & - & $\bullet$ & Andimet \\
\hline
\end{tabular}

(C) Corresponding Member $\bullet=$ Yes Blank $=$ No 


\section{Table 6 Explanatory Notes}

1. The International Laboratory Accreditation Cooperation (ILAC) is an international cooperation among 44 laboratory accreditation schemes operated throughout the world. ILAC is a forum for the development of laboratory accreditation practices and procedures, the promotion of laboratory accreditation as a trade facilitation tool, the assistance of developing accreditation systems, and the recognition of competent test facilities around the world. ILAC also provides advice and assistance to countries that are in the process of developing their own laboratory accreditation systems.

2. The International Accreditation Forum (IAF) is an organization of accreditors of certifiers, association of certifiers and internationally oriented trade associations. The purpose of IAF is to share experiences in carrying out accreditation and certifications in the use of ISO/IEC guides. IAF also seeks to establish the equivalence of the programs of its members that are accreditors.

3. The Interamerican Accreditation Cooperation (IAAC) is a regional organization of accreditation bodies from the American countries whose main purpose is to harmonize accreditation procedures and reach multilateral recognition of conformity assessment systems. IAAC accreditation includes product certification, personnel certification, quality and environmental systems registration and laboratory accreditation. IAAC supports the development of accreditation systems in any member country seeking to set one up and in compliance with the appropriate ISO/IEC international guides.

4. Note that the members of ILAC, IAF or IAAC are accreditation bodies and not countries. There may be more than one accreditation body per economy that are represented in these international bodies. 
Table 6: International Accreditation of Conformity Assessment Bodies

\begin{tabular}{|l|c|c|c|}
\hline $\begin{array}{l}\text { Accreditation } \\
\text { Bodies from: }\end{array}$ & ILAC & IAF & IAAC \\
\hline Antigua \& Barbuda & & & \\
\hline Argentina & $\bullet$ & $\bullet$ & $\bullet$ \\
\hline The Bahamas & & & \\
\hline Barbados & & & \\
\hline Belize & & & \\
\hline Bolivia & & & $\bullet$ \\
\hline Brasil & $\bullet$ & $\bullet$ & $\bullet$ \\
\hline Canada & $\bullet$ & $\bullet$ & \\
\hline Chile & & & $\bullet$ \\
\hline Colombia & & $\bullet$ & $\bullet$ \\
\hline Costa Rica & & & $\bullet$ \\
\hline Dominica & & $\bullet$ & $\bullet$ \\
\hline Ecuador & & & \\
\hline El Salvador & & & $\bullet(A)$ \\
\hline Grenada & & & $\bullet$ \\
\hline Guatemala & & & $\bullet$ \\
\hline Guyana & & & $\bullet$ \\
\hline Häiti & & & \\
\hline Honduras & & & \\
\hline Jamaica & & & \\
\hline México & & & \\
\hline Nicaragua & & & \\
\hline Panamá & & & \\
\hline Paraguay & & & \\
\hline Perú & & & \\
\hline República Dominicana & & & \\
\hline St Kitts \& Nevis & & & \\
\hline Saint Lucia & & & \\
\hline St Vincent \& Grenadines & & & \\
\hline Suriname & & & \\
\hline Trinidad \&Tobago & & & \\
\hline United States & & & \\
\hline Uruguay & & & \\
\hline Venezuela & & & \\
\hline (C) & & & \\
\hline
\end{tabular}




\section{Table 7 Explanatory Notes}

Below are the definitions for the acronyms in Table 7 in alphabetical order.

ANDEAN PACT - The Andean Pact was founded in 1969. Its members are Bolivia, Colombia, Ecuador, Perú and Venezuela. It is a customs union with an approximate population of 101 million people and an approximate GDP of US \$194 billion dollars. Its members established the Andean Committee on Standardization, Certification and Metrology (CAN) to harmonize product standards and technical regulations that comprise intraregional trade.

ALADI - The Latin American Integration Association is the oldest and broadest integration forum in Latin America. It dates back to 1960 with the creation of the Latin American Free Trade Association. Its members are Argentina, Bolivia, Brasil, Chile, Colombia, Ecuador, México, Paraguay, Perú, Uruguay and Venezuela. Its members represent $95 \%$ of the GDP, and $87 \%$ of the territory and $86 \%$ of the population of Latin America and the Caribbean. Its members have concluded more than 80 bilateral and sub-regional agreements that refer to trade and other areas.

APEC- The Asia-Pacific Economic Cooperation (APEC) was established in1989. Its goal is to reduce trade and investment barriers so that trade expands within the Asia-Pacific region and with the world, and goods, services, capital, and investment flow freely among APEC economies. The members of APEC: Australia, Brunei Darussalam, Canada, Chile, People's Republic of China, Hong Kong, China, Indonesia, Japan, Republic of Korea, Malaysia, México, New Zealand, Papua New Guinea, Perú, Republic of the Philippines, Russia, Singapore, Chinese Taipei, Thailand, United States and Vietnam. APEC has a combined GDP of over US\$16 trillion and 44 percent of global trade.

CACM - Central America Common Market was founded December 1960. It entered into force in 1973 with the Puntarenas Declaration. Its members are Costa Rica, El Salvador, Guatemala, Honduras and Nicaragua. It is a common market that seeks to harmonize monetary and fiscal policy with an approximate population of 30 million people and an approximate GDP of US\$ 3 billion dollars. Belize and Panamá participate in CACM summits but not in regional trade integration.

CARIBCAN - Caribbean-Canadian Common Market is a program for trade, investment and industrial cooperation between Canada and the Commonwealth Caribbean region that started in June 1986. Its members are: Anguilla, Antigua and Barbuda, The Bahamas, Bermuda, Barbados, Belize, British Virgin Islands, Cayman Islands, Dominica, Grenada, Guyana, Jamaica, Monsterrat, St. Kitts and Nevis, St. Lucia, St. Vincent and the Grenadines, Trinidad and Tobago, Turks and Caicos Islands and Canada.

CARICOM - Caribbean Common Market was founded in July 1973 with the signing of the Treaty of Chaguaramas. Its members are Antigua and Barbuda, The Bahamas, Barbados, Belize, Dominica, Grenada, Guyana, Jamaica, Montserrat, St Kitts and Nevis, St Lucia, St Vincent and the Grenadines, Suriname and Trinidad and Tobago. Despite its name, CARICOM is a custom union, not a common market, but eventually it hopes to become a common market. The 
approximate population of the region is 6 million people with a total GDP of approximately US $\$ 29$ billion dollars. Presently, the Caribbean Region Organization for Standards and Quality (CROSQ) is being established to address and coordinate regional standards issues and harmonize technical standards among its members.

CBI- The Caribbean Basin Initiative (CBI) is a program of the United States of America which provides for the duty free entry of merchandise from designated beneficiary countries. The initial program was enacted in 1984 and revised in 1990 with an indefinite lifetime. Its members are: Antigua \& Barbuda, The Bahamas, Barbados, Belize, British Virgin Islands, Costa Rica, Dominica, El Salvador, Grenada, Guatemala, Guyana, Häiti, Honduras, Jamaica, Montserrat, Netherlands Antilles, Nicaragua, Panamá, República Dominicana, St Kitts and Nevis, St Lucia, St Vincent and the Grenadines, Trinidad and Tobago and the United States.

G3 - The Group of Three was founded in June 1994 in Cartagena, Colombia. Its members are Colombia, México and Venezuela. It is a Free Trade Area with an approximate population of 150 million people and an approximate GDP: of US\$ 385 billion dollars. It established the Comments on Standards-Related Measures that coordinates standards activities for the members.

NAFTA - North American Free Trade Agreement was signed December 1992. It became effective January 1994. Its members are Canada, México and the United States. It is a Free Trade Area with an approximate population of 388 million people and an approximate GDP of US\$ 7117 billion dollars. It established the Committee on Standards-Related Measures with five subcommittees in the areas of transportation, telecommunications, automotive, labeling and pesticides to coordinate standards related activities.

MERCOSUR- Southern Cone Common Market was founded March 1991 with the signing of the Treaty of Asuncion. It entered into force in January 1995. Its members are Argentina, Brasil, Paraguay and Uruguay. It is a customs union and an eventual common market. The approximate population of the region is 207 million, with a total GDP of approximately US\$ 667 billion dollars. In 1991 the "MERCOSUR Standards Committee, CMN" was created to develop technical standards. CMN is the only forum recognized by MERCOSUR for the harmonization and development of voluntary standards. It is composed of the national standards bodies of the four member countries, that is IRAM (Argentina), ABNT (Brasil), INTN (Paraguay), and UNIT (Uruguay). CMN has a management council and 16 standards committees by sector where standards are harmonized or developed. 
Table 7: Membership to Regional Hemispheric Trade Agreements

\begin{tabular}{|c|c|}
\hline Country & Trade Agreements \\
\hline Antigua \& Barbuda & CARIBCAN, CARICOM, CBI \\
\hline Argentina & MERCOSUR, ALADI \\
\hline The Bahamas & CARIBCAN, CARICOM, CBI \\
\hline Barbados & CARIBCAN, CARICOM, CBI \\
\hline Belize & CARIBCAN, CARICOM, CACM*, CBI \\
\hline Bolivia & ANDEAN PACT, G3, ALADI \\
\hline Brasil & MERCOSUR, ALADI \\
\hline Canada & APEC, CARIBCAN, NAFTA \\
\hline Chile & $\begin{array}{l}\text { ALADI, APEC bilaterals with Colombia, } \\
\text { Ecuador, México, Venezueala }\end{array}$ \\
\hline Colombia & ANDEAN PACT, ALADI, G3 \\
\hline Costa Rica & CACM, CBI \\
\hline Dominica & CARIBCAN, CARICOM, CBI \\
\hline Ecuador & ANDEAN PACT, ALADI \\
\hline El Salvador & CACM, CBI \\
\hline Grenada & CARIBCAN, CARICOM, CBI \\
\hline Guatemala & CARICOM, CBI \\
\hline Guyana & CARIBCAN, CARICOM, CBI \\
\hline Häiti & CARIBCAN, CARICOM, CBI \\
\hline Honduras & CACM, CBI \\
\hline Jamaica & CARIBCAN, CARICOM, CBI \\
\hline México & ALADI, APEC, G3, NAFTA \\
\hline Nicaragua & CACM, CBI \\
\hline Panamá & $\mathrm{CACM}^{*}, \mathrm{CBI}$ \\
\hline Paraguay & ALADI, MERCOSUR \\
\hline Perú & ALADI, ANDEAN PACT, APEC \\
\hline República Dominicana & CARICOM, CBI \\
\hline St Kitts \& Nevis & CARIBCAN, CARICOM, CBI \\
\hline Saint Lucia & CARIBCAN, CARICOM, CBI \\
\hline St Vincent \&Grenadines & CARIBCAN, CARICOM, CBI \\
\hline Suriname & CARICOM \\
\hline Trinidad \& Tobago & CARIBCAN, CARICOM, CBI \\
\hline United States & APEC, CBI, NAFTA \\
\hline Uruguay & ALADI, MERCOSUR \\
\hline Venezuela & ALADI, ANDEAN PACT, G3 \\
\hline
\end{tabular}

* See CACM description 
Table 8: Conformity Assessment Infrastructure

\begin{tabular}{|c|c|c|c|c|}
\hline Country & $\begin{array}{c}\text { Inspection } \\
\text { And } \\
\text { Testing }\end{array}$ & $\begin{array}{c}\text { Product } \\
\text { Certification }\end{array}$ & $\begin{array}{c}\text { Quality } \\
\text { Systems } \\
\text { Registration }\end{array}$ & $\begin{array}{c}\text { Laboratory } \\
\text { Accreditation }\end{array}$ \\
\hline Antigua \& Barbuda & - & & & \\
\hline Argentina & - & - & $\bullet$ & $\bullet$ \\
\hline The Bahamas & - & - & & \\
\hline Barbados & - & - & & \\
\hline Belize & ni & ni & ni & ni \\
\hline Bolivia & - & $\bullet$ & & \\
\hline Brasil & 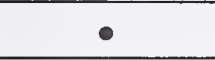 & - & $\bullet$ & - \\
\hline Canada & - & 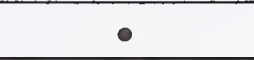 & - & - \\
\hline Chile & $\bullet$ & $\bullet$ & $\bullet$ & $\bullet$ \\
\hline Colombia & - & - & - & - \\
\hline Costa Rica & $\bullet$ & $\bullet$ & $\bullet$ & $\cdot$ \\
\hline Dominica & - & & & \\
\hline Ecuador & $\bullet$ & $\bullet$ & $\bullet$ & \\
\hline EI Salvador & - & & & \\
\hline Grenada & $\bullet$ & & & \\
\hline Guatemala & - & - & & \\
\hline Guyana & - & - & & - \\
\hline Häiti & - & $\bullet$ & & \\
\hline Honduras & $\bullet$ & & & \\
\hline Jamaica & $\bullet$ & $\bullet$ & & $\bullet$ \\
\hline México & $\bullet$ & $\bullet$ & $\bullet$ & $\bullet$ \\
\hline Nicaragua & - & & & \\
\hline Panamá & - & - & $\bullet$ & - \\
\hline Paraguay & $\bullet$ & $\bullet$ & & \\
\hline Perú & $\bullet$ & $\bullet$ & $\bullet$ & - \\
\hline República Dominicana & $\bullet$ & - & $\bullet$ & $\bullet$ \\
\hline St Kitts \& Nevis & ni & ni & ni & ni \\
\hline Saint Lucia & - & - & & \\
\hline St Vincent \& Grenadines & ni & ni & ni & ni \\
\hline Suriname & $\bullet$ & & & \\
\hline Trinidad \&Tobago & - & $\bullet$ & 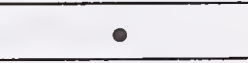 & \\
\hline United States & $\bullet$ & $\bullet$ & 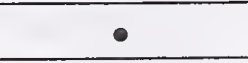 & $\cdot$ \\
\hline Uruguay & $\bullet$ & $\bullet$ & $\bullet$ & \\
\hline Venezuela & - & $\bullet$ & - & $\bullet$ \\
\hline
\end{tabular}


Table 9: Quality System Registration

\begin{tabular}{|c|c|}
\hline Country & $\begin{array}{c}\text { Companies registered } \\
\text { ISO } 9000\end{array}$ \\
\hline Antigua \& Barbuda & 1 \\
\hline Argentina & 524 \\
\hline The Bahamas & 0 \\
\hline Barbados & 6 \\
\hline Belize & ni \\
\hline Bolivia & 1 \\
\hline Brasil & 2476 \\
\hline Canada & 3000 \\
\hline Chile & 65 \\
\hline Colombia & 130 \\
\hline Costa Rica & 25 \\
\hline Dominica & 0 \\
\hline Ecuador & ni \\
\hline El Salvador & 5 \\
\hline Grenada & 1 \\
\hline Guatemala & 3 \\
\hline Guyana & 3 \\
\hline Häiti & 0 \\
\hline Honduras & 0 \\
\hline Jamaica & 7 \\
\hline México & 192 \\
\hline Nicaragua & 1 \\
\hline Panamá & 15 \\
\hline Paraguay & 3 \\
\hline Perú & 20 \\
\hline República Dominicana & 21 \\
\hline St. Kitts \& Nevis & ni \\
\hline Saint Lucia & 1 \\
\hline St. Vincent \& Grenadines & $\overline{\text { ni }}$ \\
\hline Suriname & 0 \\
\hline Trinidad \&Tobago & 17 \\
\hline United States & 24014 \\
\hline Uruguay & 17 \\
\hline Venezuela & 162 \\
\hline
\end{tabular}

ni $=$ no information 


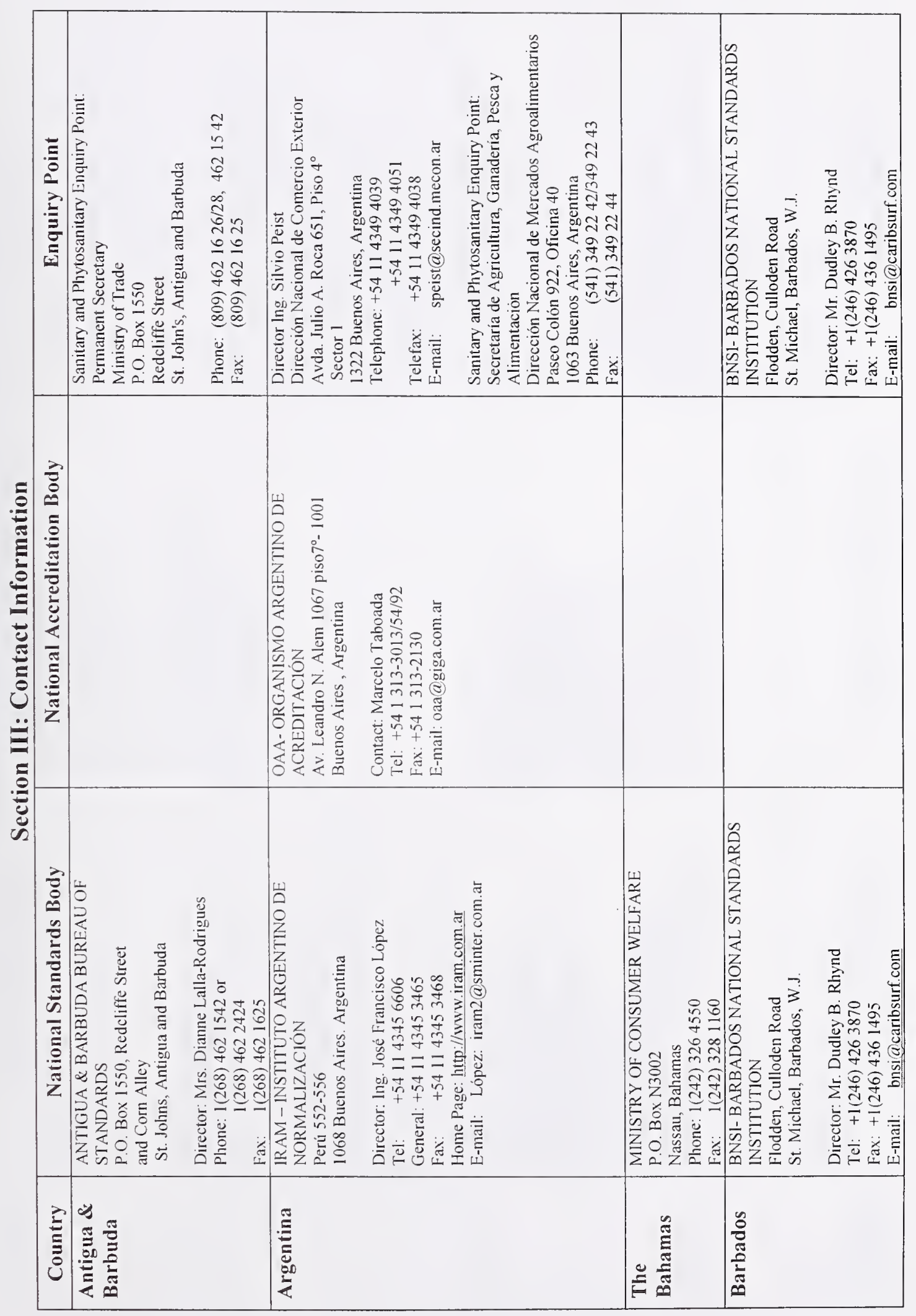




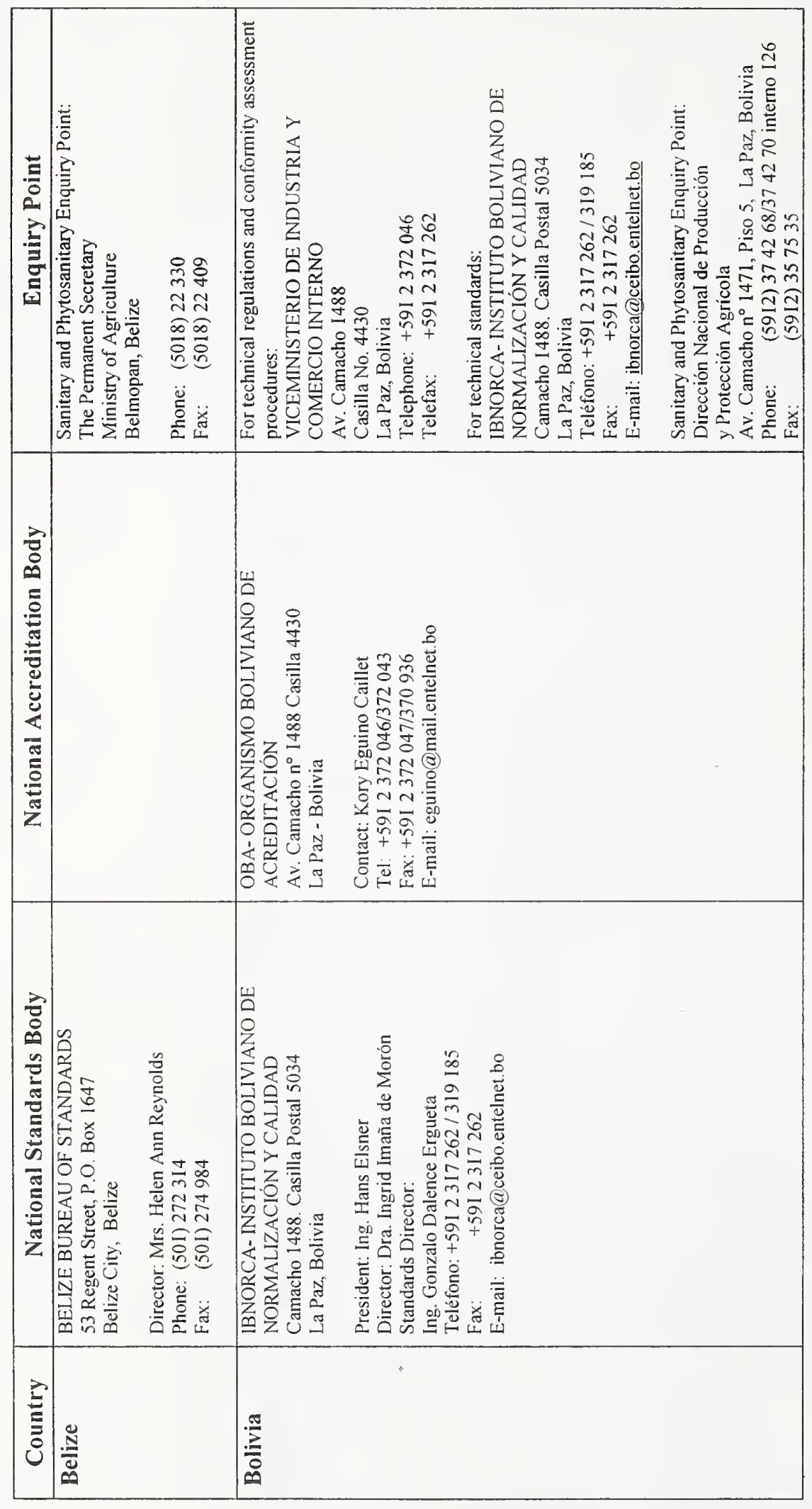




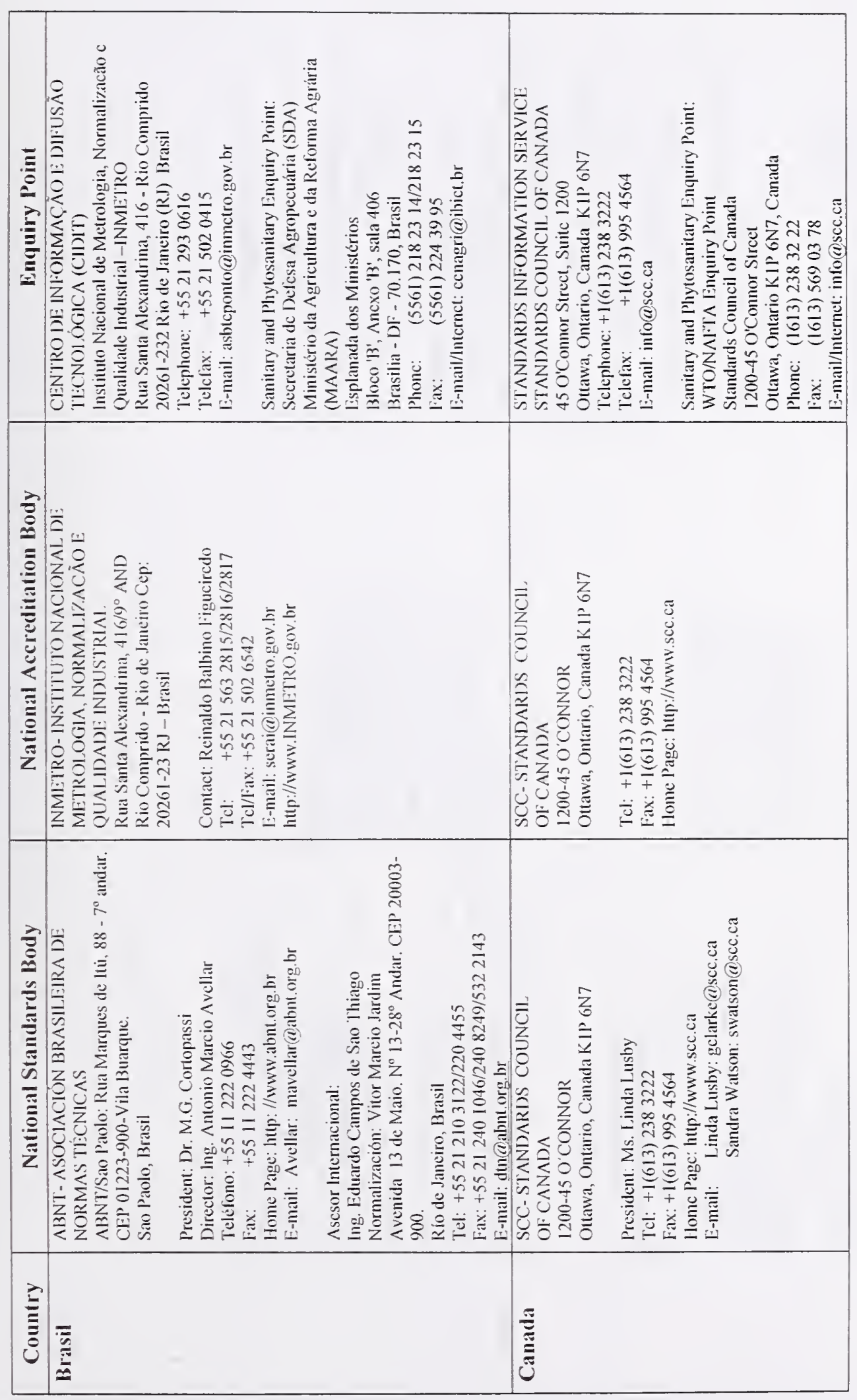




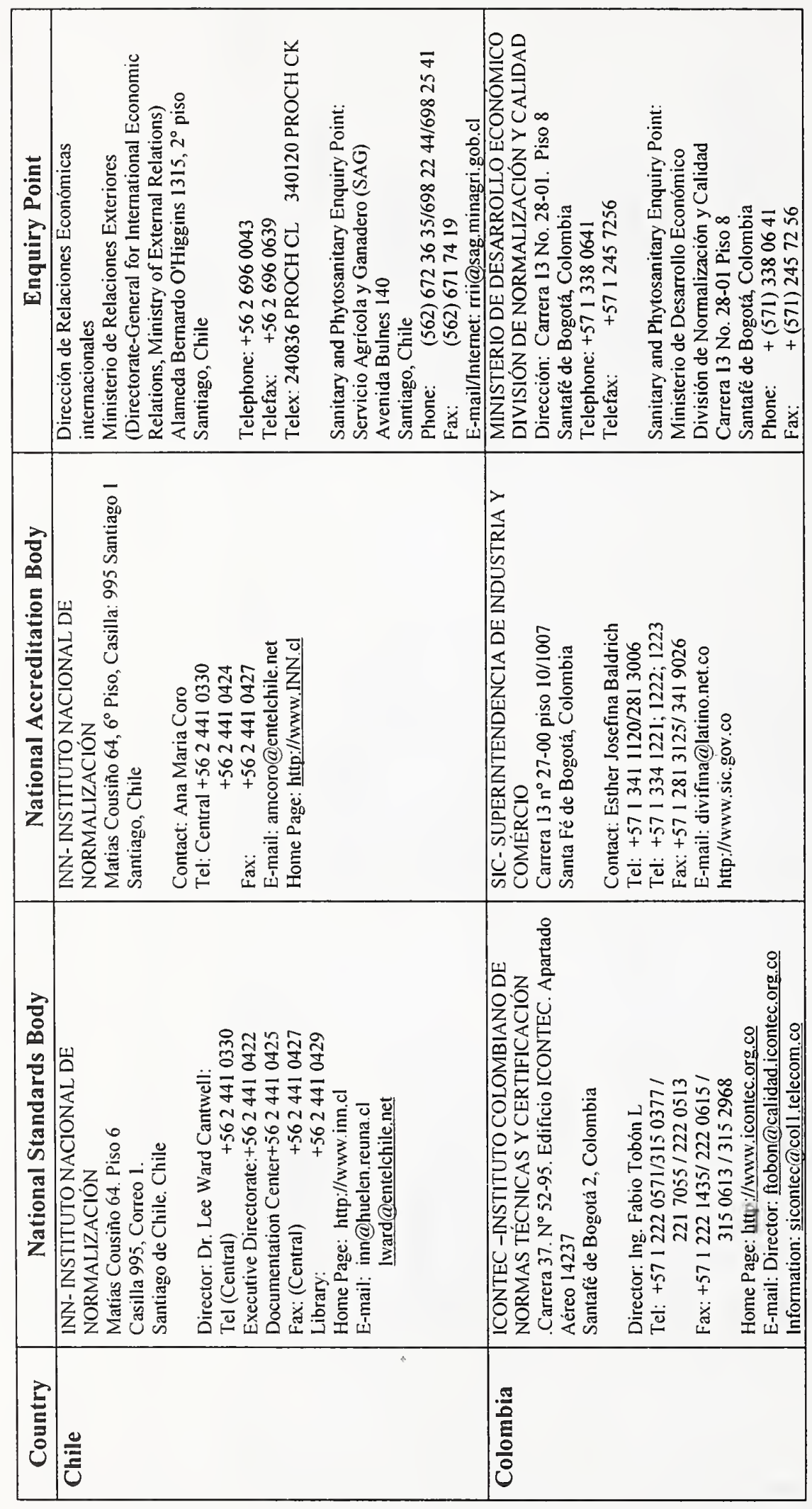




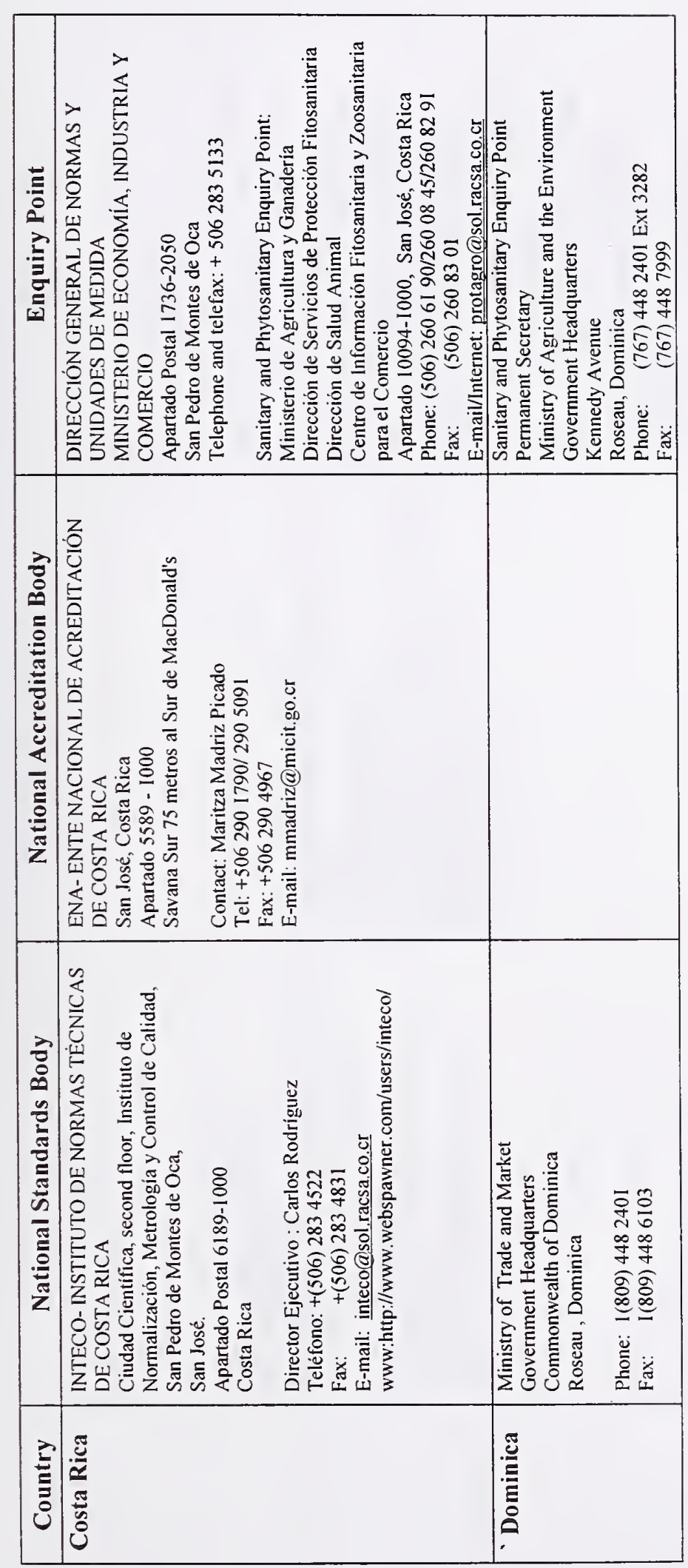




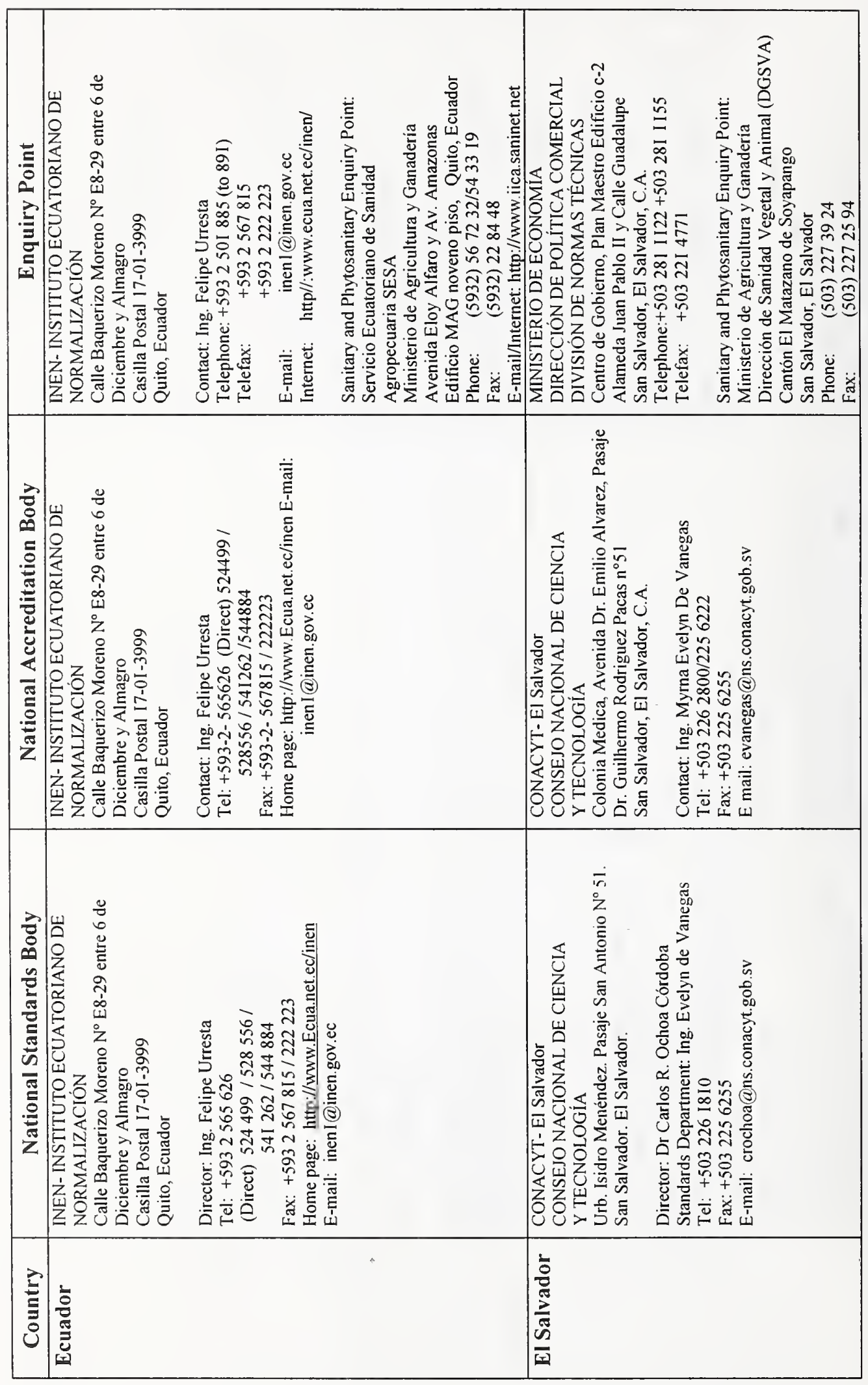




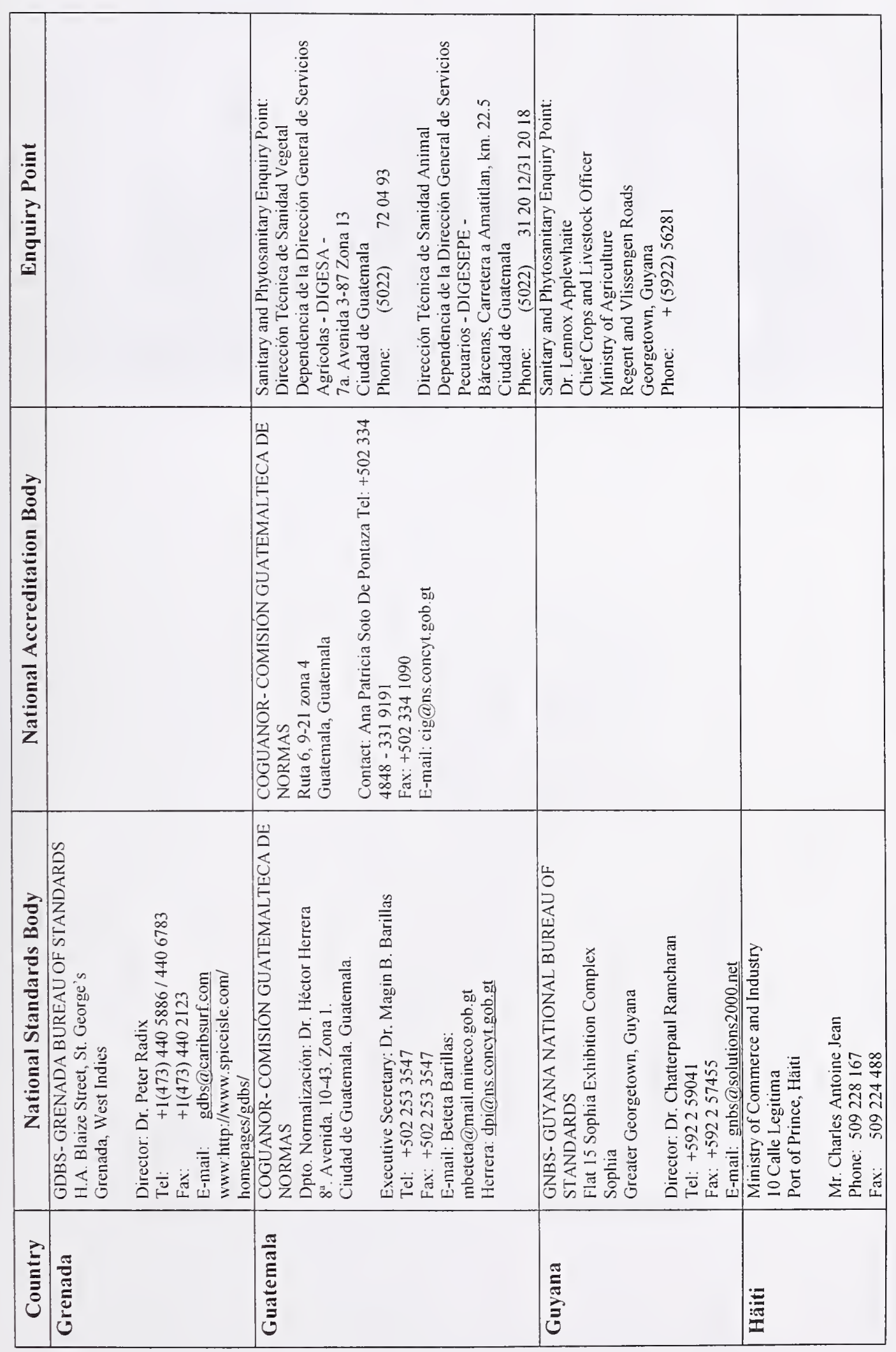




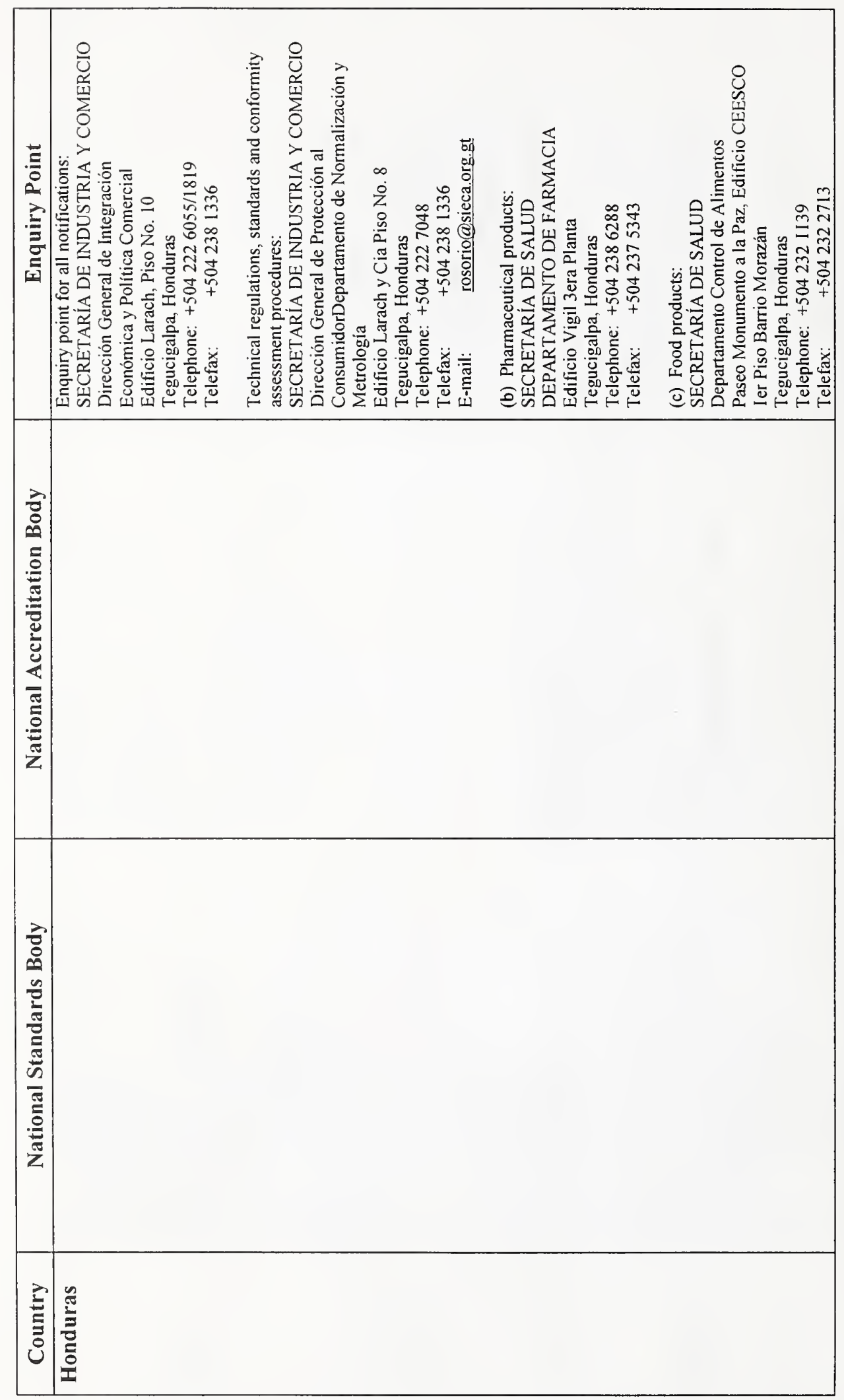




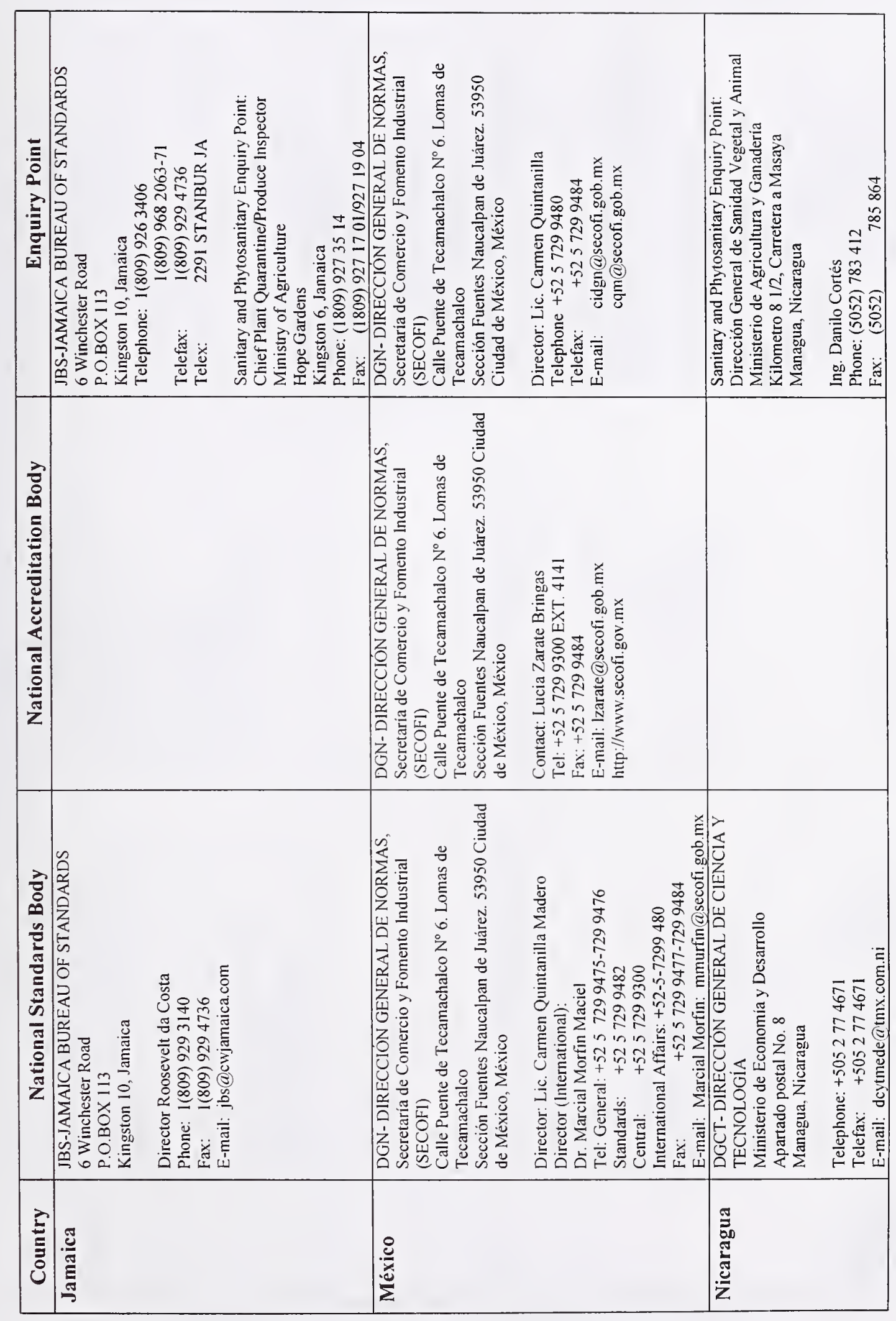




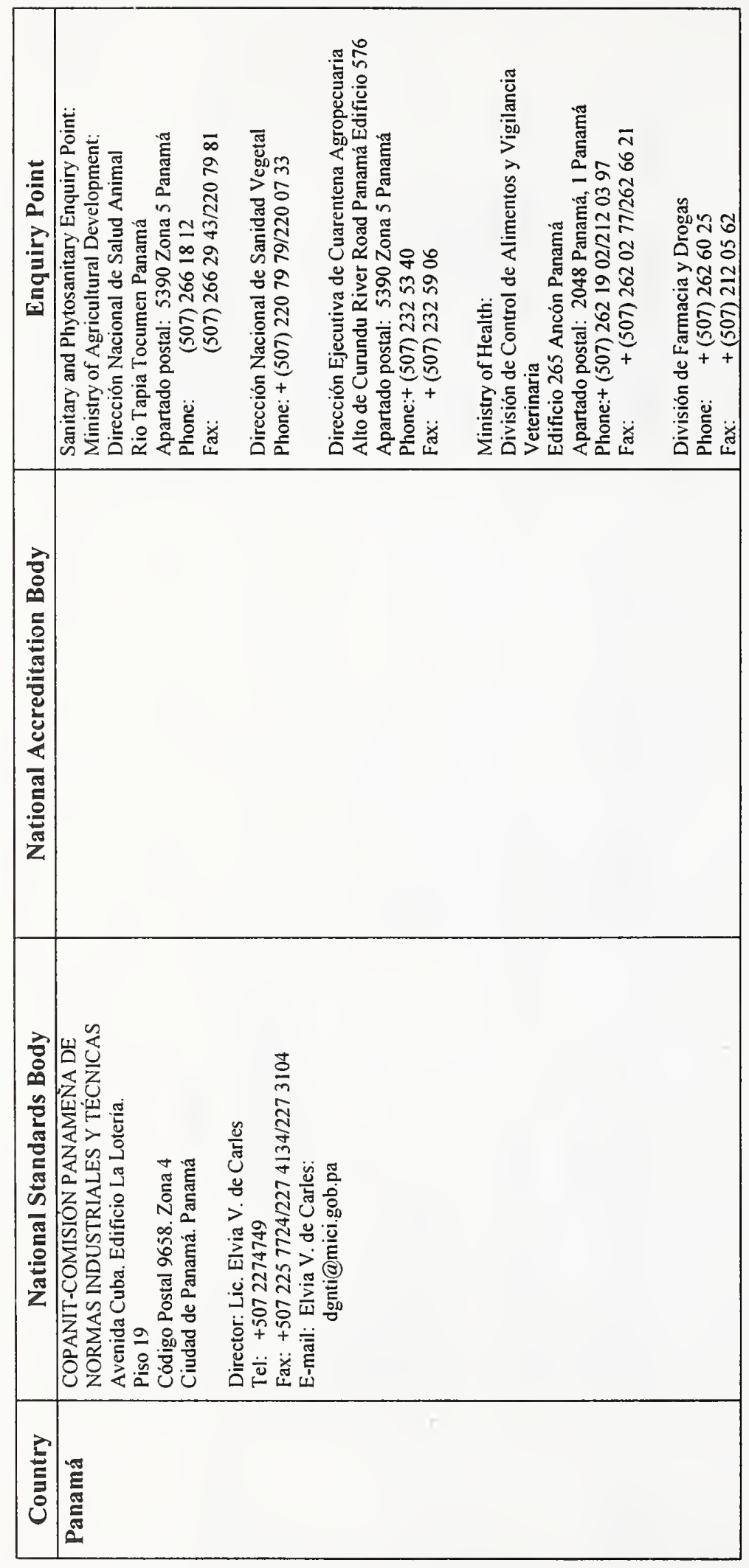




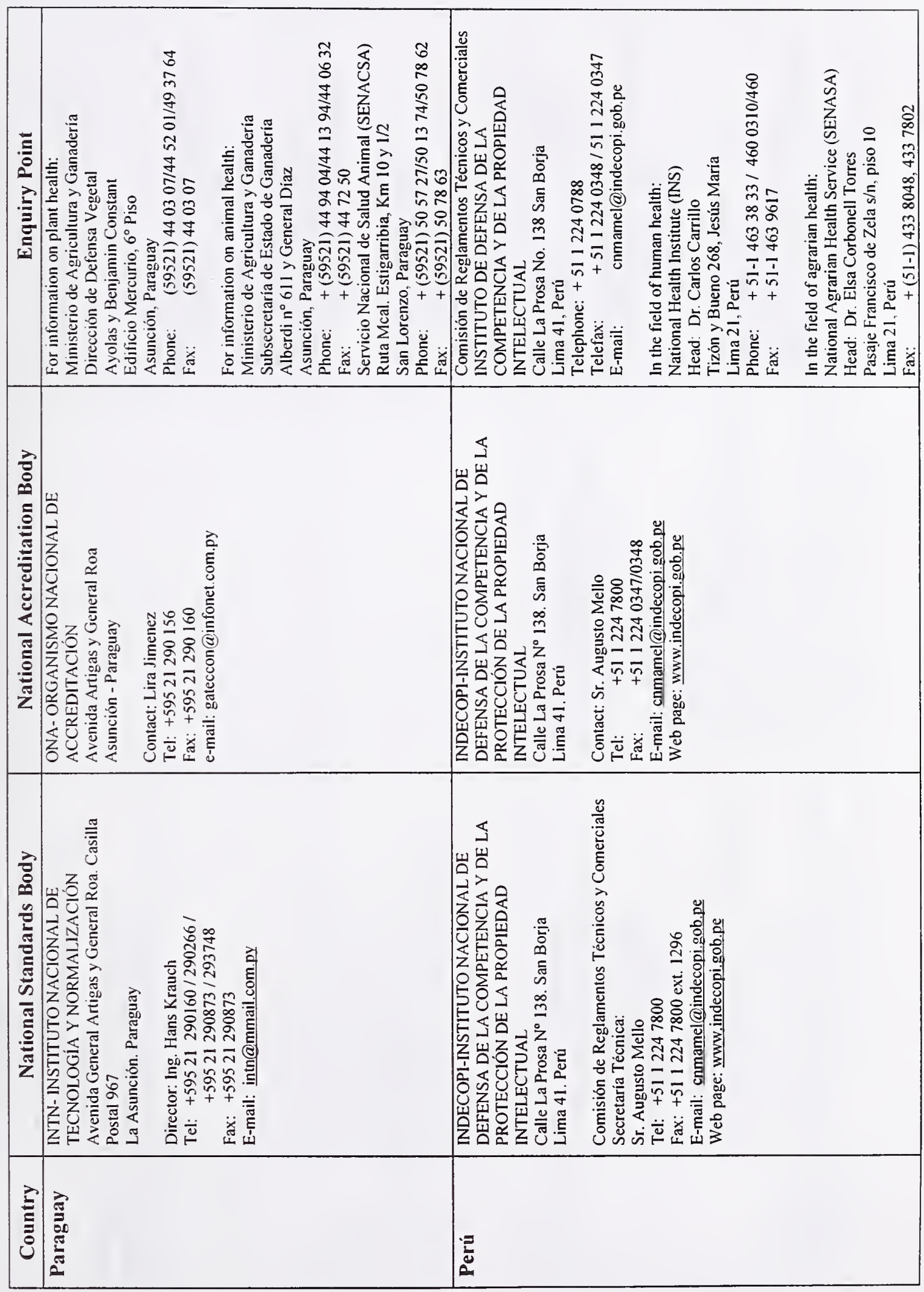




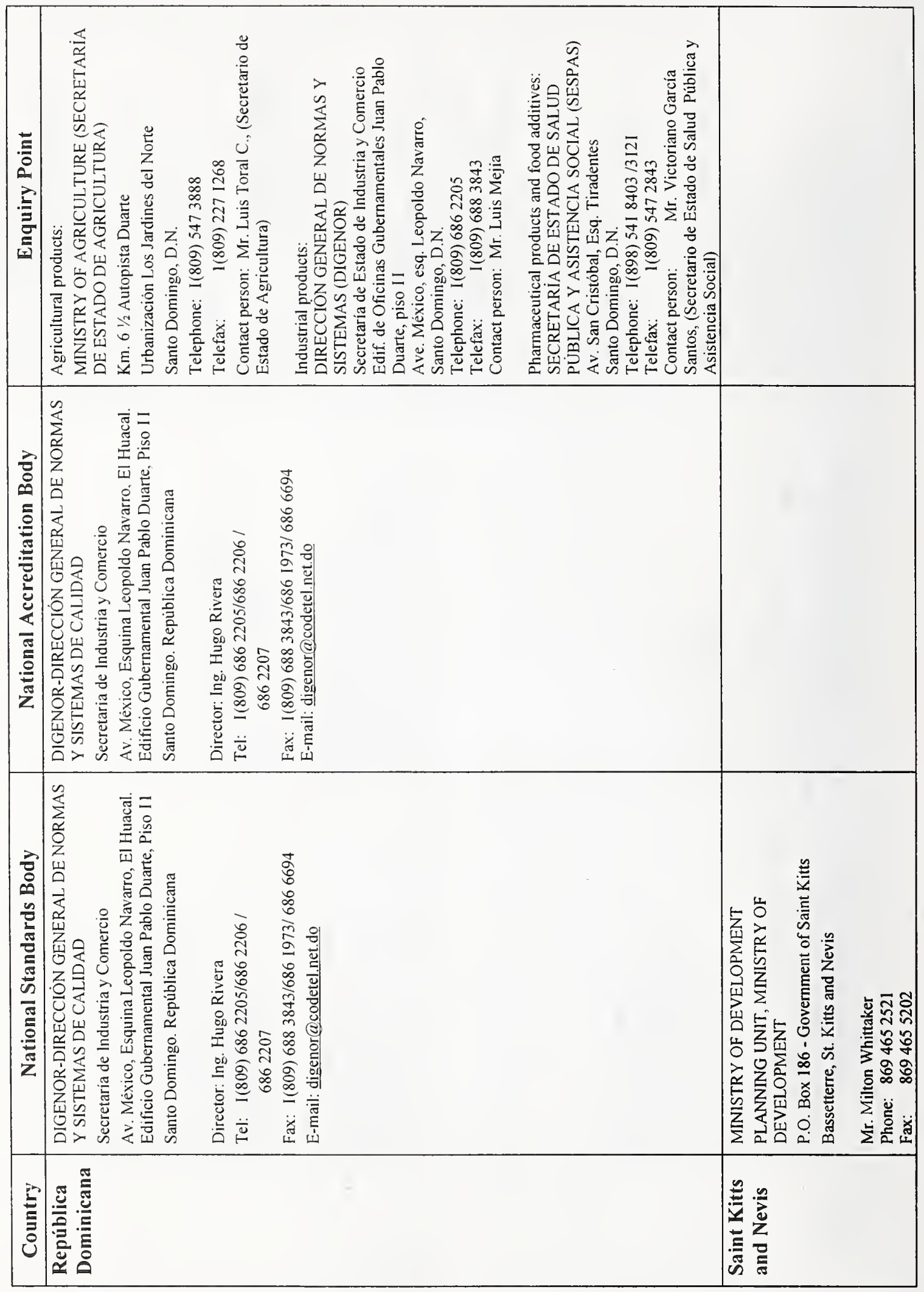




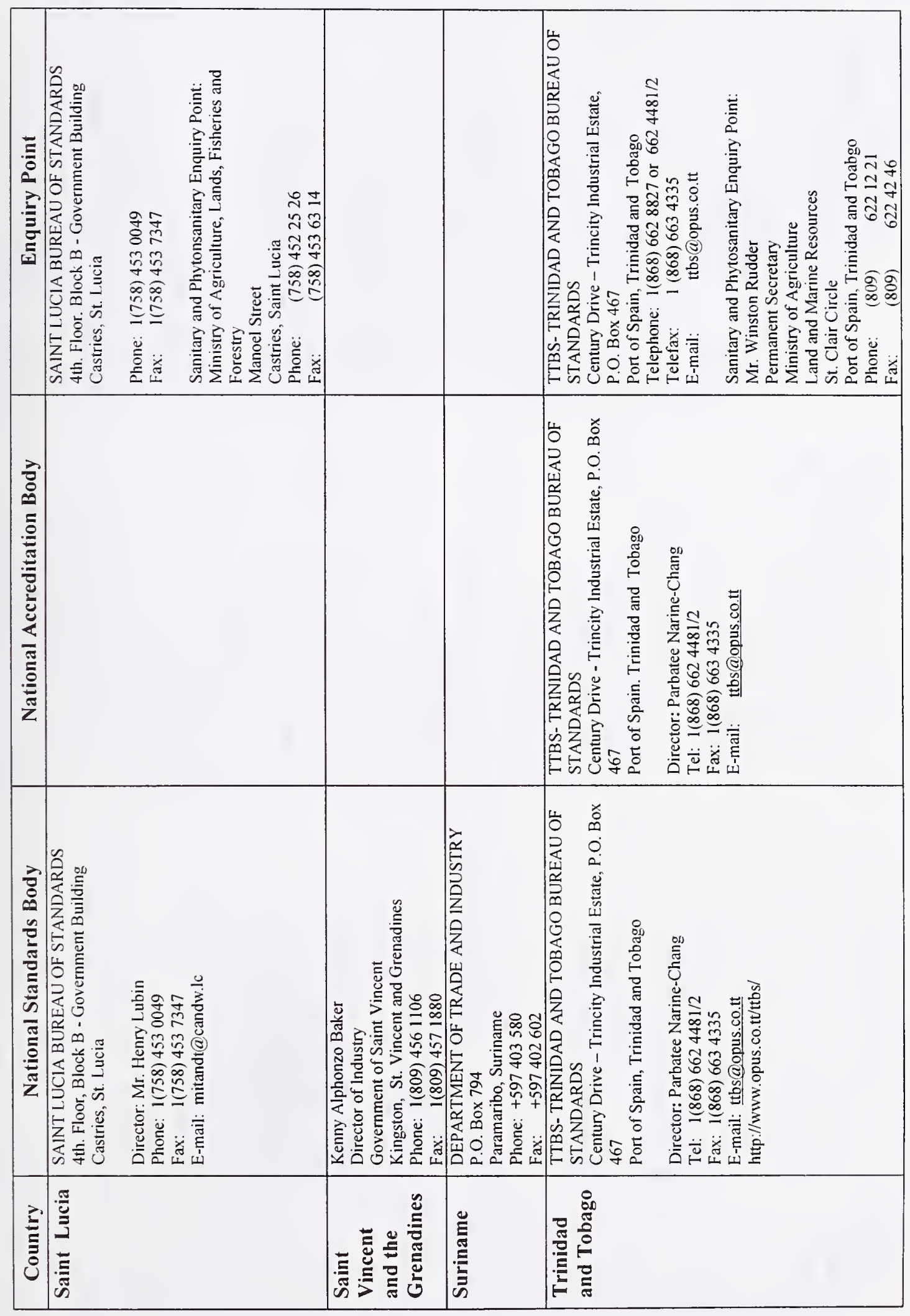




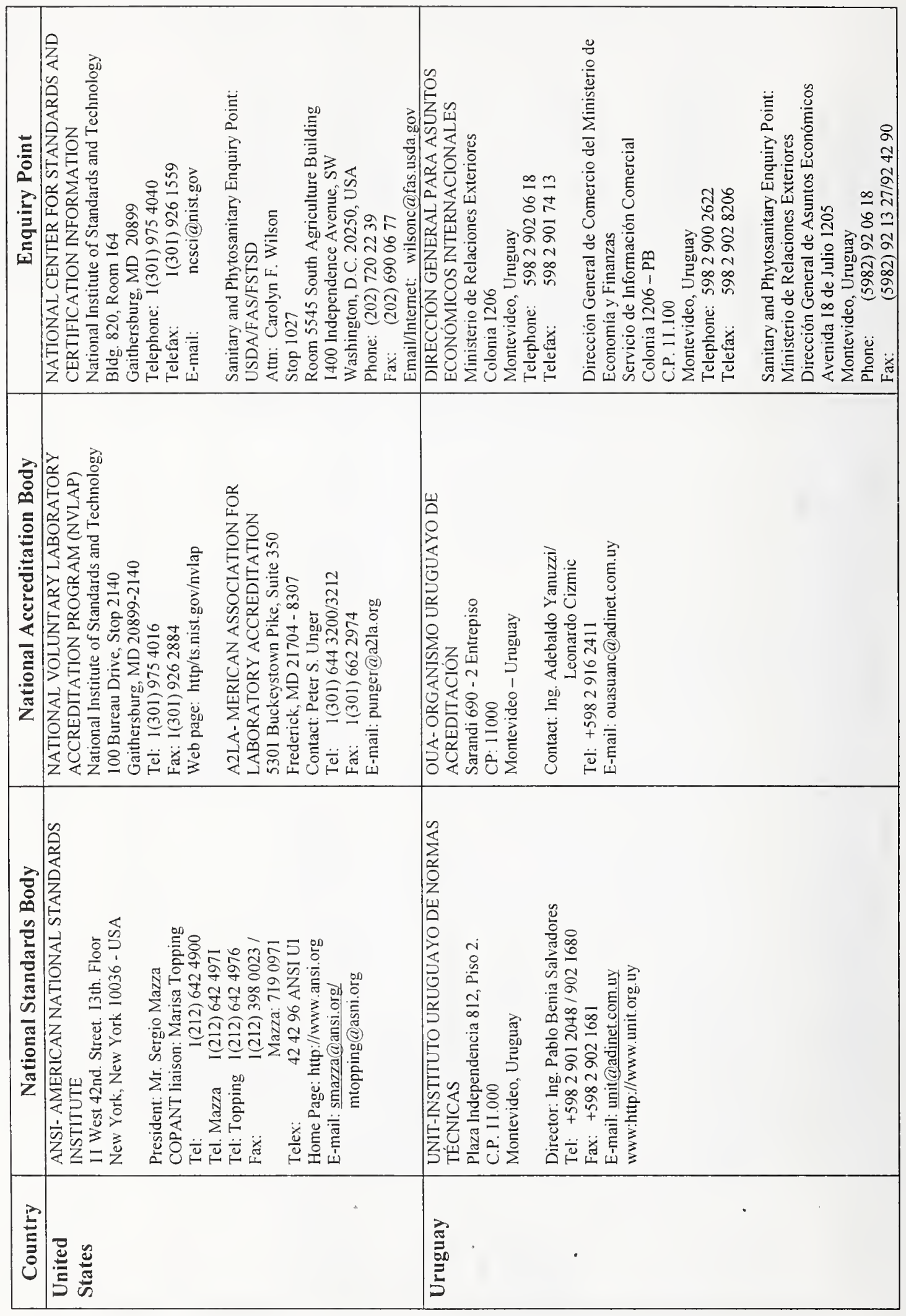




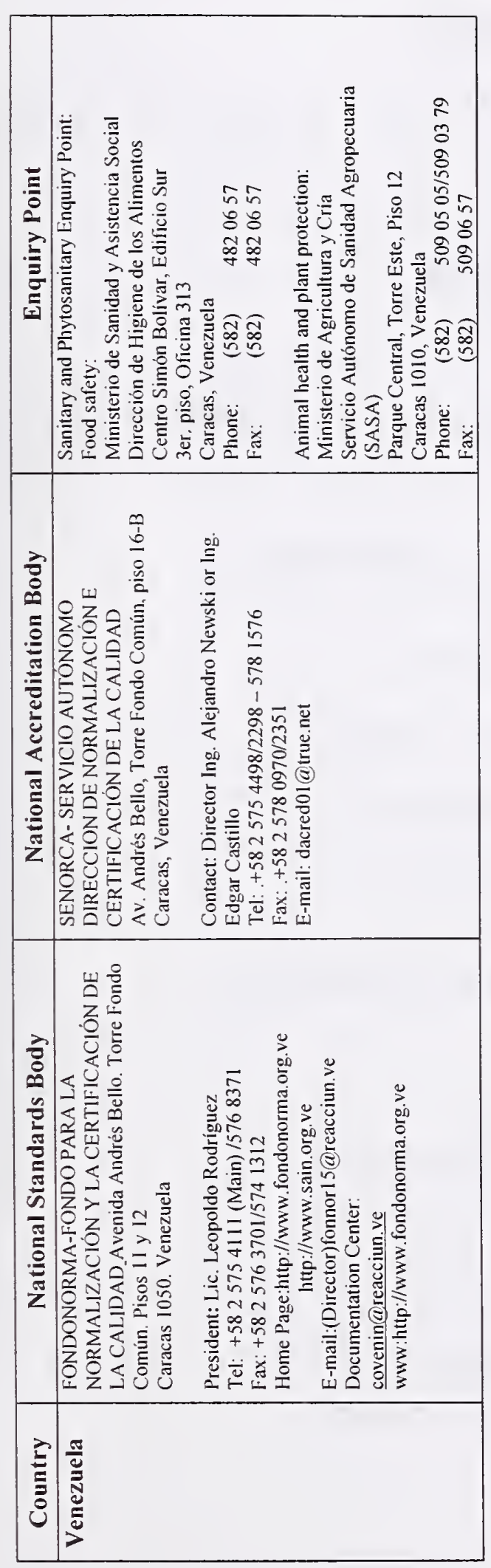




\section{Useful Web Sites}

\begin{tabular}{|c|c|}
\hline ALADI & $\begin{array}{l}\text { Latin American Integration Association } \\
\text { http://www.aladi.org/ }\end{array}$ \\
\hline \multirow[t]{2}{*}{ APEC } & Asia Pacific Economic Cooperation \\
\hline & http://www.apecsec.org.sg/ \\
\hline \multirow[t]{2}{*}{ BIPM } & International Bureau of Weights and Measures \\
\hline & http://www.bipm.fr/enus/welcome.html \\
\hline \multirow[t]{2}{*}{$\mathrm{CMN}$} & MERCOSUR Standardization Committee \\
\hline & http://www.cmn.org.br \\
\hline \multirow[t]{2}{*}{ CODEX } & Codex Alimentarius Commission \\
\hline & http://www.fao.org/waicent/faoinfo/economic/esn/codex/Default.htm \\
\hline \multirow[t]{2}{*}{ COPANT } & Pan American Standards Commission \\
\hline & http://www.copant.org/ \\
\hline \multirow[t]{2}{*}{ FTAA } & Free Trade Area of the Americas \\
\hline & http://www.alca-ftaa.org/EnglishVersion/alca_e.htm \\
\hline \multirow[t]{2}{*}{ IAAC } & InterAmerican Accreditation Cooperation \\
\hline & http://www.ibpinetsp.com.br/iaac/ \\
\hline \multirow[t]{2}{*}{ IEC } & International Electrotechnical Commission \\
\hline & http://www.iec.ch/home-e.htm \\
\hline \multirow[t]{2}{*}{ ILAC } & International Laboratory Accreditation Cooperation \\
\hline & http://www.ilac.org/ \\
\hline \multirow[t]{2}{*}{ ISO } & International Organization for Standardization \\
\hline & http://www.iso.ch/ \\
\hline \multirow[t]{2}{*}{ ITU } & International Telecommunications Union \\
\hline & http://www.itu.int/home/index.html \\
\hline \multirow[t]{2}{*}{ MERCOSUR } & Southern Cone Common Market \\
\hline & http://www.americasnet.com/mauritz/mercosur/english/ \\
\hline \multirow[t]{2}{*}{ OAS } & Organization of American States \\
\hline & http://www.oas.org/ \\
\hline \multirow[t]{2}{*}{ OAS-SA } & OAS Summit of the Americas \\
\hline & http://www.summit-americas.org/ \\
\hline \multirow[t]{2}{*}{ OIML } & International Organization of Legal Metrology \\
\hline & http://www.oiml.org/ \\
\hline \multirow[t]{2}{*}{ PASC } & Pacific Area Standards Congress \\
\hline & http://www.pascnet.org/ \\
\hline \multirow[t]{2}{*}{ SIM } & InterAmerican Metrology System \\
\hline & http://www.ibpinet.com.br/sim/ \\
\hline \multirow[t]{2}{*}{ NORAMET } & North America Cooperation in Metrology \\
\hline & http://www.nrc.ca/inms/int_coop/noramet.html \\
\hline \multirow[t]{2}{*}{ WTO } & World Trade Organization \\
\hline & http://www.wto.org/wto/ \\
\hline
\end{tabular}




\section{Selected Acronyms}

$\begin{array}{ll}\text { ANDEAN PACT } & \text { Andean Pact Trade Agreement } \\ \text { ANDIMET } & \text { Andean Region Metrology, subregion of SIM } \\ \text { ALADI } & \text { Latin American Integration Association } \\ \text { APEC } & \text { Asia Pacific Economic Cooperation } \\ \text { BIPM } & \text { International Bureau of Weights and Measures } \\ \text { CAMET } & \text { Central America Metrology Subregion of SIM } \\ \text { CAN } & \text { Andean Committee on Standardization, Certification and Metrology } \\ \text { CACM } & \text { Central America Common Market } \\ \text { CARIBCAN } & \text { Caribbean-Canadian Common Market } \\ \text { CARICOM } & \text { Caribbean Common Market } \\ \text { CARIMET } & \text { Caribbean Metrology Subregion of SIM } \\ \text { CBI } & \text { Caribbean Basin Initiative } \\ \text { CCMSC } & \text { Caribbean Common Market Standards Council } \\ \text { CMN } & \text { MERCOSUR Standardization Committee } \\ \text { CODEX } & \text { Codex Alimentarius Commission } \\ \text { COPANT } & \text { Pan American Standards Commission } \\ \text { CSRM } & \text { Committee on Standards-Related Measures } \\ \text { FTAA } & \text { Free Trade Area of the Americas } \\ \text { G3 } & \text { Group of Three Trade Agreement } \\ \text { IAAC } & \text { Interamerican Accreditation Cooperation } \\ \text { IAF } & \text { International Accreditation Forum } \\ \text { IEC } & \text { International Electrotechnical Commission } \\ \text { ILAC } & \text { International Laboratory Accreditation Cooperation } \\ \text { ISO } & \text { International Organization for Standardization } \\ \text { ISONET } & \text { ISO Information Network } \\ \text { ITU } & \text { International Telecommunications Union } \\ \text { MERCOSUR } & \text { Southern Cone Common Market } \\ \text { NORAMET } & \text { North America Metrology, subregion of SIM } \\ \text { NAFTA } & \text { North America Free Trade Agreement } \\ \text { OAS } & \text { Organization of American States } \\ \text { OIML } & \text { International Organization of Legal Metrology } \\ \text { PASC } & \text { Pacific Area Standards Congress } \\ \text { SIM } & \text { Interamerican Metrology System } \\ \text { SURAMET } & \text { South American Metrology, subregion of SIM } \\ \text { WTO } & \text { World Trade Organization } \\ \text { TBT } & \text { Technical Barriers to Trade Agreement } \\ & \end{array}$




\section{Appendix A: Bibliography}

1. AENOR, National Standards and Certification Body of Spain", "Certificación Miembros de COPANT, excepto Canadá y Estados Unidos", Madrid, (1996).

2. COPANT, Panamerican Standards Commission, "Member Institutes", Venezuela, (1997)

3. COPANT, Panamerican Standards Commission, "Resultados de la Segunda Encuesta Sobre Certificación y Acreditamiento de Laboratorios", Venezuela, (1994).

4. Fernandes, María, Visiers Ricardo (1997), "Misión de identificación en Paraguay, Uruguay, Argentina y Brasil del 13 de Enero al 5 de Febrero de 1997 Informe", Portugal, (1997). Report prepared for the European Union as a Mercosur Program on Technical Standards.

5. INMETRO, National Institute of Metrology, Standardization and Industrial Quality, "Manual para Empresas Exportadoras sobre Barreras Técnicas ao Comércio Internacional", Rio de Janeiro, (1997).

6. Martinez, Guillermo, "Standards and Certification for the electrical Sector in Latin America", NEMA, National Electrical Manufacturers Association Spring Conference, Florida, U.S. (1998).

7. Martinez, Ileana, "Standards and Conformity Assessment in Argentina", International Market Insight (IMI), Brazil, (1999), "Standards and Conformity Assessment in Chile", (1999), Standards and Conformity Assessment in Brazil", (1998).

8. OAS Trade Unit, "National Practices on Standards, Technical Regulations and Conformity Assessment in the Western Hemisphere", Washington, D.C. (1997). Inventory prepared for the Free Trade Area of the Americas Working Group on Standards and Technical Barriers to Trade.

9. PLANCAL, Plan Andino de la Calidad, Lima, Perú, (1997). Study financed by the European Union and prepared by the Spanish Quality Association and the technical experts of the Standards, Quality and Technical Regulations Unit of the Junta del Acuerdo de Cartagena.

10. Presentations made by Argentina, Bolivia, Central America, Colombia, Costa Rica, Cuba, Chile, México, Panamá, Paraguay, Perú, República Dominicana, Uruguay and Venezuela, at the "Seminario de Normalización y Certificación Eléctrica de Iberoamérica", México, (1997).

11. Stephenson, Sherry M., "Standards, the Environment and Trade Facilitation in the Western Hemisphere: Negotiating in the FTAA", Washington, D.C. (1997).

12. Toth, Robert B., "Profiles of National Standards-Related Activities", NIST Special Publication 912, Gaithersburg, M.D., (1997).

13. Underwriters Laboratory, "Conformity Assessment Analysis Argentina, Brazil, Chile, Colombia, Perú, and Venezuela", North Carolina, U.S., (1998).

14. Interamerican Accreditation Cooperation, "Survey on Conformity Assessment Structure of the 11 ALADI Member Countries", Rio de Janeiro, Brasil, (1999). 
Appendix B: Surveys

Conformity Assessment Survey for the FTAA countries

Country:

Inspection and Testing

1) Does your country have any inspection and testing facilities used for regulatory control? (Please include University facilities if appropriate)

YES

NO

Approximately, how many are PUBLIC?

Approximately, how many are PRIVATE?

2) Please give examples of the three most important sectors inspection and testing facilities serve:

1.

2.

3.

3) Does your country have a body or system for the accreditation of inspection and testing facilities?

YES

NO

Approximately how many inspection and testing facilities have been accredited?

4) Does your country accept testing and inspection results issued by bodies in:

other countries?

YES

NO

the United States?

YES

$\mathrm{NO}$ 


\section{Product Certification}

1) Does your country have a National Body for the certification of products used for regulatory control?

YES NO

2).What is the name the National Product Certification Body?

Is it Public? Or Private?

Please give examples of the three most important sectors the National Product Certification Body serves:

1.

2.

3

2) Whether or not your country has a National Product Certification Body, please answer this question. Does your country have any other product certification bodies?

YES NO

Are these product certification bodies PUBLIC?

YES NO

Approximately how many public bodies are there?

Are these product certification bodies PRIVATE?

YES NO

Approximately how many private bodies are there? 
Please name the three most important sectors where these product certification bodies (public or private) operate?

1.

2.

3.

4) Does your country have a body or system for the accreditation of entities that perform product certification?

YES

NO

Approximately how many product certifiers have been accredited?

5) Are there transnational or international bodies that operate product certification programs in your country?

YES

$\mathrm{NO}$

Approximately how many are there?

Please give two examples of international bodies that operate product certification programs in your country:

1.

2.

6) Does your country perform product certification activities in the name of international or transnational bodies?

YES

NO

Please give examples.

1.

2.

7) Does your country accept product certification results issued by bodies in: other countries?

YES

$\mathrm{NO}$

the United States?

YES

$\mathrm{NO}$ 


\section{Quality System Certification/Registration}

1) Does your country have a National Body for the Certification/Registration of Quality Systems used for regulatory control?

YES

NO

2) What is the name the National Body for the Certification/Registration of Quality Systems?

Is it Public? Or Private?

Please give examples of the three most important sectors the National Body for the Certification/Registration of Quality Systems serve:

1.

2.

3

3) Whether or not your country has a National Body for the Certification/Registration of Quality Systems, please answer this question. Does your country have any other bodies for the Certification/Registration of Quality Systems?

YES

NO

Are these bodies PUBLIC?

YES

NO

Approximately how many public bodies are there?

Are these bodies PRIVATE?

YES

NO

Approximately how many bodies are there?

Please name the three most important sectors where these bodies (public or private) operate?

1.

2.

3. 
4). Does your country have a body or system for the accreditation of entities that perform certification/ registration of quality systems?

YES

$\mathrm{NO}$

Approximately how many quality systems certifiers/registrars have been accredited?

5) Are there transnational or international bodies that operate Certification/Registration of Quality Systems in your country?

YES

$\mathrm{NO}$

Approximately how many are there?

Please give two examples of international bodies that operate quality systems certification/registration programs in your country.

1.

2.

6) Does your country perform quality systems certification/registration activities in the name of international or transnational bodies?

YES

$\mathrm{NO}$

Please give two examples.

1.

2.

7) Approximately how many companies have had their quality system certified/registered in your country?

8) Does your country accept quality system certification/registration results issued by bodies in: other countries?

YES

$\mathrm{NO}$

the United States?

YES

$\mathrm{NO}$ 
1) Does your country have a National Program for Laboratory Accreditation used for regulatory control?

YES

NO

2) What is the name of the National Program for Laboratory Accreditation?

Is it Public?

Or Private?

Please give examples of the three most important sectors the National Program for Laboratory Accreditation serves:

1.

2.

3

3). For the National Program for Laboratory Accreditation:

How many calibration laboratories have been accredited?

How many testing laboratories have been accredited?

4) Whether or not your country has a National Program for Laboratory Accreditation please answer this question. Does your country have any other Laboratory Accreditation programs?

YES NO

Are these laboratory accreditation programs PUBLIC?

YES NO

Approximately how many public programs are there?

Are these laboratory accreditation programs PRIVATE?

YES

$\mathrm{NO}$

Approximately how many private programs are there? 
Please name the three most important sectors where these laboratory accreditation programs (public or private) operate?

1.

2.

3.

5) Are there transnational or international bodies that operate laboratory accreditation programs in your country?

YES

$\mathrm{NO}$

Approximately how many are there?

Please give two examples of international bodies that operate laboratory accreditation programs in your country:

1.

2.

6) Does your country perform laboratory accreditation activities in the name of international or transnational bodies?

YES

NO

Please give two examples.

1.

2.

7) Approximately how many laboratories have been accredited in your country, whether they were accredited by the National Program for Laboratory Accreditation or by any other programs in your country national or international?

Total number of accredited calibration laboratories?

Total number of accredited testing laboratories?

8) Does your country accept laboratory accreditation results issued by laboratory accreditation programs in:

other countries?

YES

NO

the United States?

YES

NO 


\section{Encuesta sobre la Evaluación de la Conformidad de los países del ALCA}

País:

Inspección y Ensayo

1) ¿Cuenta su país con entidades de inspección, ensayos o pruebas que operan en el campo reglamentado? (Por favor incluya entidades universitarias si es el caso).

SI NO

Aproximadamente, ¿cuántas son PÚBLICAS?

Aproximadamente, ¿cuántas son PRIVADAS?

2) Por favor enumere los tres sectores de más importancia en que estas entidades operan:

1.

2.

3.

3) ¿Cuenta su país con un organismo o sistema para la acreditación de entidades de inspección, ensayo o prueba?
SI
NO

Aproximadamente, ¿cuántas entidades de inspección, ensayo o prueba han sido acreditadas?

4) ¿Acepta su país los resultados de inspecciones, ensayos o pruebas de otros países?

¿De otros países?

SI

NO

¿De los Estados Unidos? SI

NO 


\section{Certificación de Productos}

1) ¿Cuenta su país con una Entidad Nacional para la Certificación de Productos que opera en el campo reglamentado?

SI

NO

2) ¿Cuál es el nombre de la Entidad Nacional para la Certificación de Productos?

¿Es esta entidad Pública?

O Privada?

Por favor enumere los tres sectores de más importancia en que esta entidad opera:

1.

2.

3.

3) Ya sea que su país cuente o no con una Entidad Nacional para la Certificación de Productos, por favor conteste esta pregunta. ¿Tiene su país otras entidades para la certificación de productos??

$\mathrm{SI}$

¿Son éstas entidades PÚBLICAS?

$\mathrm{SI}$

Aproximadamente, ¿cuántas entidades públicas existen?

¿Son éstas entidades PRIVADAS?

SI

$\mathrm{NO}$

Aproximadamente, ¿cuántas entidades privadas existen?

Por favor enumere los tres sectores de más importancia en que estas entidades (públicas o privadas) operan:

1.

2.

3. 
4) ¿Cuenta su país con un organismo o sistema para la acreditación de entidades para la la certificación de productos?

SI

NO

¿Aproximadamente, cuántas entidades para la certificación de productos han sido acreditadas?

5) ¿Existen en su país entidades transnacionales o internacionales que operan como certificadores de productos?

SI

NO

Aproximadamente, ¿cuántas hay?

Por favor dé dos ejemplos de entidades extranjeras que operan en su país como certificadores de productos:

1.

2.

6) ¿Desempeña su país actividades para la certificación de productos en nombre de entidades transnacionales o internacionales?

SI $\quad$ NO

Por favor dé dos ejemplos.

1.

2.

7) ¿Acepta su país las certificaciones de productos de otros países?

¿De otros países?

SI

NO

¿De los Estados Unidos? SI

NO 


\section{Certificación/Registración de Sistemas de Calidad}

1) ¿Cuenta su país con una Entidad Nacional para la Certificación/Registración de Sistemas de Calidad que opera en el campo reglamentado?

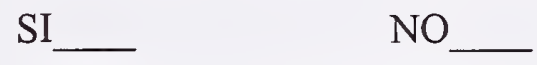

2) ¿Cuál es el nombre de la Entidad Nacional para la Certificación/Registración de Sistemas de Calidad?

¿Es esta entidad Pública? ___ O Privada?

Por favor enumere los tres sectores de más importancia en que esta entidad opera:

1.

2.

3.

3) Ya sea que su país cuente o no con una Entidad Nacional para la Certificación/ Registración de Sistemas de Calidad, por favor conteste esta pregunta. ¿Tiene su país otras entidades para la Certificación/Registración de Sistemas de Calidad?

SI__ NO__

¿Son éstas entidades PÚBLICAS? SI__ NO

Aproximadamente, ¿cuántas entidades públicas existen?

¿Son éstas entidades PRIVADAS?

SI__ NO

Aproximadamente, ¿cuántas entidades privadas existen?

Por favor enumere los tres sectores de más importancia en que estas entidades (públicas o privadas) operan:

1.

2.

3. 
4) ¿Cuenta su país con un organismo o sistema para la acreditación de entidades para la la Certificación/Registración de Sistemas de Calidad?

$$
\text { SI }
$$

NO

¿Aproximadamente, cuántas entidades para la Certificación/Registración de Sistemas de Calidad han sido acreditadas?

5) ¿Existen en su país entidades transnacionales o internacionales que operan como certificadores/registradores de Sistemas de Calidad?

SI

$\mathrm{NO}$

Aproximadamente, ¿cuántas hay?

Por favor dé dos ejemplos de entidades extranjeras que operan en su país como certificadores/registradores de Sistemas de Calidad:

1.

2.

6) ¿Desempeña su país actividades para la Certificación/Registración de Sistemas de Calidad en nombre de entidades transnacionales o internacionales?

SI $\mathrm{NO}$

Por favor dé dos ejemplos.

1.

2.

7) ¿Aproximadamente, cuántas compañías o entidades han recibido certificados o registros de sus sistemas de calidad?

8) ¿Acepta su país las certificaciones/registros de sistemas de calidad de otros países?

¿De otros países?

SI

$\mathrm{NO}$

¿De los Estados Unidos? SI

$\mathrm{NO}$ 


\section{Acreditación de Laboratorios}

1) ¿Cuenta su país con un Programa Nacional de Acreditación de Laboratorios que opera en el campo reglamentado?

SI NO

2) ¿Cuál es el nombre del Programa Nacional de Acreditación de Laboratorios?

¿Es este programa Público? O Privado?

Por favor enumere los tres sectores de más importancia en que opera este prograna:

1.

2.

3.

3) El Programa Nacional de Acreditación de Laboratorios ha acreditado:

¿cuántos laboratorios de calibración?

¿cuántos laboratorios de ensayo o prueba?

4) Ya sea que su país cuente o no con un Programa Nacional de Acreditación de Laboratorios, por favor conteste esta pregunta. ¿Tiene su país otros programas para la acreditación de laboratorios?

SI__ NO

¿Son éstos programas PÚBLICOS?

SI $\quad$ NO

¿Aproximadamente cuántos programas públicos existen?

¿Son éstos programas PRIVADOS?

SI

$\mathrm{NO}$

Aproximadamente, cuántos programas privados existen? 
Por favor enumere los tres sectores de más importancia en que estos programas (públicos o privados) operan:

1.

2.

3.

5) ¿Existen en su país entidades transnacionales o internacionales que operan como acreditadores de laboratorios?

SI

$\mathrm{NO}$

Aproximadamente, ¿cuántos hay?

Por favor dé dos ejemplos de entidades extranjeras que operan en su país como acreditadores de laboratorios:

1.

2.

6) ¿Desempeña su país actividades de acreditación de laboratorios en nombre de entidades transnacionales o internacionales?

SI

$\mathrm{NO}$

Por favor dé dos ejemplos.

1 .

2.

7). ¿Aproximadamente cuantos laboratorios han sido acreditados en su país ya sea por el Programa Nacional de Acreditación de Laboratorios o por otros programas de acreditación de laboratorios nacionales o internacionales?

¿Número total de laboratorios de calibración acreditados?

¿Número total de laboratorios de ensayo o prueba acreditados?

8) ¿Acepta su país las acreditaciones de laboratorios de otros países?

¿De otros países?

SI

$\mathrm{NO}$

¿De los Estados Unidos? SI

NO 




\section{NTST Technical Publications}

\section{Periodical}

Journal of Research of the National Institute of Standards and Technology-Reports NIST research and development in those disciplines of the physical and engineering sciences in which the Institute is active. These include physics, chemistry, engineering, mathematics, and computer sciences. Papers cover a broad range of subjects, with major emphasis on measurement methodology and the basic technology underlying standardization. Also included from time to time are survey articles on topics closely related to the Institute's technical and scientific programs. Issued six times a year.

\section{Nonperiodicals}

Monographs-Major contributions to the technical literature on various subjects related to the Institute's scientific and technical activities.

Handbooks-Recommended codes of engineering and industrial practice (including safety codes) developed in cooperation with interested industries, professional organizations, and regulatory bodies.

Special Publications-Include proceedings of conferences sponsored by NIST, NIST annual reports, and other special publications appropriate to this grouping such as wall charts, pocket cards, and bibliographies.

National Standard Reference Data Series-Provides quantitative data on the physical and chemical properties of materials, compiled from the world's literature and critically evaluated. Developed under a worldwide program coordinated by NIST under the authority of the National Standard Data Act (Public Law 90-396). NOTE: The Journal of Physical and Chemical Reference Data (JPCRD) is published bimonthly for NIST by the American Chemical Society (ACS) and the American Institute of Physics (AIP). Subscriptions, reprints, and supplements are available from ACS, 1155 Sixteenth St., NW, Washington, DC 20056.

Building Science Series-Disseminates technical information developed at the Institute on building materials, components, systems, and whole structures. The series presents research results, test methods, and performance criteria related to the structural and environmental functions and the durability and safety characteristics of building elements and systems.

Technical Notes - Studies or reports which are complete in themselves but restrictive in their treatment of a subject. Analogous to monographs but not so comprehensive in scope or definitive in treatment of the subject area. Often serve as a vehicle for final reports of work performed at NIST under the sponsorship of other government agencies.

Voluntary Product Standards-Developed under procedures published by the Department of Commerce in Part 10, Title 15, of the Code of Federal Regulations. The standards establish nationally recognized requirements for products, and provide all concerned interests with a basis for common understanding of the characteristics of the products. NIST administers this program in support of the efforts of private-sector standardizing organizations.

Order the following NIST publications-FIPS and NISTIRs—from the National Technical Information Service, Springfield, VA 22161.

Federal Information Processing Standards Publications (FIPS PUB)--Publications in this series collectively constitute the Federal Information Processing Standards Register. The Register serves as the official source of information in the Federal Government regarding standards issued by NIST pursiant to the Federal Property and Administrative Services Act of 1949 as amended, Public Law 89-306 (79 Stat. 1127), and as implemented by Executive Order 11717 (38 FR 12315, dated May 11, 1973) and Part 6 of Title 15 CFR (Code of Federal Regulations).

NIST Interagency or Internal Reports (NISTIR)-The series includes interim or final reports on work performed by NIST for outside sponsors (both government and nongovernment). In general, initial distribution is handled by the sponsor; public distribution is handled by sales through the National Technical Information Service, Springfield, VA 22161, in hard copy, electronic media, or microfiche form. NISTIR's may also report results of NIST projects of transitory or limited interest, including those that will be published subsequently in more comprehensive form. 


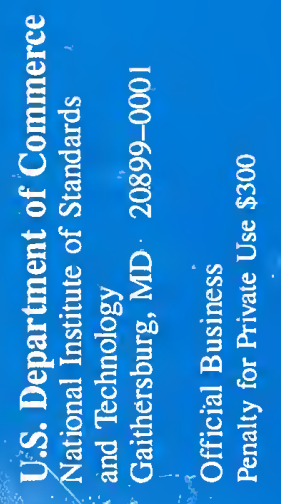

